\title{
A WALK IN THE NONCOMMUTATIVE GARDEN
}

\author{
ALAIN CONNES AND MATILDE MARCOLLI
}

\section{Contents}

\begin{tabular}{|c|c|}
\hline 1. Introduction & 2 \\
\hline 2. Handling noncommutative spaces in the wild: basic tools & 2 \\
\hline 3. Phase spaces of microscopic svstems & 6 \\
\hline 4. Noncommutative quotients & 9 \\
\hline 5. Spaces of leaves of foliations & 10 \\
\hline 6. The noncommutative tori & 17 \\
\hline 7. Duals of discrete groups & 22 \\
\hline 8. Brillouin zone and the quantum Hall effect & 24 \\
\hline 9. Tilings & 29 \\
\hline 10. Noncommutative spaces from dvnamical svstems & 32 \\
\hline 11. Noncommutative spaces from string theorv & 36 \\
\hline 12. Groupoids and the index theorem & 38 \\
\hline 13. Riemannian manifolds. conical singularities & 39 \\
\hline 14. Cantor sets and fractals & 45 \\
\hline 15. Spaces of dimension $z$ and DimReg & 48 \\
\hline 16. Local algebras in supersvmmetric QFT & 50 \\
\hline 17. Spacetime and the standard model of elementary particles & 51 \\
\hline 18. Isospectral deformations & 57 \\
\hline 19. Algebraic deformations & 59 \\
\hline 20. Quantum groups & 62 \\
\hline 21. Spherical manifolds & 66 \\
\hline 22. O-lattices & 69 \\
\hline 23. Modular Hecke algebras & 74 \\
\hline 24. Noncommutative moduli spaces. Shimura varieties & 81 \\
\hline 25. The adeles class space and the spectral realization & 84 \\
\hline 26. Thermodvnamics of endomotives and the Tehran program & 90 \\
\hline References & 100 \\
\hline
\end{tabular}




\section{INTRODUCTION}

If you cleave the hearth of one drop of water a hundred pure oceans emerge from it.

(Mahmud Shabistari, Gulshan-i-raz)

We have decided to contribute to the volume of the IPM lectures on noncommutative geometry a text that collects a list of examples of noncommutative spaces. As the quote of the Sufi poet here above suggests, it is often better to approach a new subject by analyzing specific examples rather than presenting the general theory. We hope that the diversity of examples the readers will encounter in this text will suffice to convince them of the fact that noncommutative geometry is a very rich field in rapid evolution, full of interesting and yet unexplored landscapes. Many of the examples collected here have not yet been fully explored from the point of view of the general guidelines we propose in Section 2 and the main point of this text is to provide a great number of open questions. The reader should interpret this survey as a suggestion of possible interesting problems to investigate, both in the settings described here, as well as in other examples that are available but did not fit in this list, and in the many more that still await to be discovered. Besides the existing books on NCG such as [53, 144, [54, 126], 136] 103] 149, two new books are being written: one by the two authors of this paper [77, and one by Connes and Moscovici [87.

\section{Handling nOnCOMmUtative SPACES in the Wild: BasiC TOOlS}

We are going to see in many example how one obtains the algebra of coordinates $\mathcal{A}$ of a noncommutative space $X$. Here we think of $\mathcal{A}$ as being the algebra of "smooth functions", which will usually be a dense subalgebra of a $C^{*}$-algebra $\overline{\mathcal{A}}$.

Here are some basic steps that one can perform in order to acquire a good understanding of a given noncommutative space $X$ with algebra of coordinates $\mathcal{A}$.

1) Resolve the diagonal of $\mathcal{A}$ and compute the cyclic cohomology.

2) Find a geometric model of $X$ up to homotopy.

3) Construct the spectral geometry $(\mathcal{A}, \mathcal{H}, D)$.

4) Compute the time evolution and analyze the thermodynamics.

1) The first step means finding a resolution of the $\mathcal{A}$-bimodule $\mathcal{A}$ by projective $\mathcal{A}$-bimodules making it possible to compute the Hochschild homology of $\mathcal{A}$ effectively. In general, such resolutions will be of Kozsul type and the typical example is the resolution of the diagonal for the algebra $C^{\infty}(X)$ of smooth functions on a compact manifold as in the $C^{\infty}$ version 58 . of the Hochschild, Kostant, Rosenberg theorem ( $c f$. [117]). It makes it possible to know what is the analogue of differential forms and of de Rham currents on the space $X$ and to take the next step of computing the cyclic homology and cyclic cohomology of $\mathcal{A}$, which are the natural replacements for the de Rham theory. For foliation algebras this was done long ago (cf. [56, 34, 93]). It ties in with the natural double complex of transverse currents.

It is not always easy to perform this step of finding a resolution and computing Hochschild and cyclic (co)homology. For instance, in the case of algebras given by generators and relations this uses the whole theory of Kozsul duality, which has been successfully extended to $N$ homogeneous algebras ( $c f$. .98, .99], [100, 101], 102], 22]). 
One specific example in which it would be very interesting to resolve the diagonal is the modular Hecke algebras (Section 23). In essence, finding a resolution of the diagonal in the algebra of modular forms of arbitrary level, equivariant with respect to the action of the group $\mathrm{GL}_{2}\left(\mathbb{A}_{f}\right)$ of finite adeles, would yield formulas for the compatibility of Hecke operators with the algebra structure. This is a basic and hard problem of the theory of modular forms.

Cyclic cohomology (and homology) is a well developed theory which was first designed to handle the leaf spaces of foliations as well as group rings of discrete groups ( $c f$. [126]). The theory admits a purely algebraic version which is at center stage in "algebraic" noncommutative geometry, but it is crucial in the analytic set-up to construct cyclic cocycles with good compatibility properties with the topology of the algebra. For instance, when the domain of definition of the cocycle is a dense subalgebra stable under holomorphic functional calculus, it automatically gives an invariant of the $K$-theory of the underlying $C^{*}$-algebra ( $c f$. [56]).

2) The essence of the second step is that many noncommutative spaces defined as a "bad quotients" ( $c f$. Section 4) can be desingularized, provided one is ready to work up to homotopy. Thus for instance if the space $X$ is defined as the quotient

$$
X=Y / \sim
$$

of an "ordinary" space $Y$ by an equivalence relation $\sim$ one can often find a description of the same space $X$ as a quotient

$$
X=Z / \sim
$$

where the equivalence classes are now contractible spaces. The homotopy type of $Z$ is then uniquely determined and serves as a substitute for that of $X$ (see [15]).

For instance, if the equivalence relation on $Y$ comes from the free action of a torsion free discrete group $\Gamma$, the space $Z$ is simply a product over $\Gamma$ of the form

$$
Z=Y \times_{\Gamma} E \Gamma,
$$

where $E \Gamma$ is a contractible space on which $\Gamma$ acts freely and properly.

The main point of this second step is that it gives a starting point for computing the $K$-theory of the space $X$ i.e. of the $C^{*}$-algebra $A=\overline{\mathcal{A}}$ playing the role of the algebra of continuous functions on $X$. Indeed, for each element of the $K$-homology of the classifying space $Z$, there is a general construction of an index problem for "families parameterized by $X$ " that yields an assembly map (cf. [15])

$$
\mu: K_{*}(Z) \rightarrow K_{*}(A)
$$

This Baum-Connes map is an isomorphism in a lot of cases (with suitable care of torsion, $c f$. 16]) including all connected locally compact groups, all amenable groupoids and all hyperbolic discrete groups. It thus gives a computable guess for the $K$-theory of $X$.

The next step is not only to really compute $K(A)$ but also to get a good model for the "vector bundles" on $X$ i.e. the finite projective modules over $A$. This step should then be combined with the above first step to compute the Chern character using connections, curvature, and eventually computing moduli spaces of Yang-Mills connections as was done for instance for the NC-torus in 88.

3) The third step makes it possible to pass from the soft part of differential geometry to the harder "Riemannian" metric aspect. The sought for spectral geometry $(\mathcal{A}, \mathcal{H}, D)$ has three essential features: 
- The $K$-homology class of $(\mathcal{A}, \mathcal{H}, D)$.

- The smooth structure.

- The metric.

One should always look for a spectral triple whose $K$-homology class is as non-trivial as possible. Ideally it should extend to a class for the double algebra $\mathcal{A} \otimes \mathcal{A}^{o}$ and then be a generator for Poincaré duality. In general this is too much to ask for, since many interesting spaces do not fulfill Poincaré duality. The main tool for determining the stable homotopy class of the spectral triple is Kasparov's bivariant $K K$-theory. Thus it is quite important to already have taken step 2 and to look for classes whose pairing with $K$-theory is as nontrivial as can be. For the smooth structure, there is often a natural guess for a subalgebra $A^{\infty} \subset A$ of the $C^{*}$-algebra $A=\overline{\mathcal{A}}$ that will play the role of the algebra of smooth functions. It should in general contain the original algebra $\mathcal{A}$ but should have the further property of being stable under the holomorphic functional calculus. This ensures that the inclusion $A^{\infty} \subset A$ is an isomorphism in $K$-theory and makes it possible to complete the classification of smooth vector bundles.

The role of the unbounded operator $D$ for the smooth structure is that it defines the geodesic flow by the formula

$$
F_{t}(a)=e^{i t|D|} a e^{-i t|D|}, \quad \forall a \in A^{\infty}
$$

and one expects that smoothness is governed by the smoothness of the operator valued map $\mathbb{R} \ni t \mapsto F_{t}(a)$. The main result of the general theory is the local index formula of [81, which provides the analogue of the Pontrjagin classes of smooth manifolds in the noncommutative framework.

The problem of determining $D$ from the knowledge of the $K$-homology class is very similar to the choice of a connection on a bundle. There are general results that assert the existence of an unbounded selfadjoint $D$ with bounded commutators with $\mathcal{A}$ from estimates on the commutators with the phase $F$. The strongest is obtained ( $c f$. [54]) just assuming that the $[F, a]$ are in an ideal called $\mathrm{Li} \mathcal{H}$ and it ensures the existence of a theta-summable spectral triple which is what one needs to get started.

It is not always possible to find a finitely-summable spectral triple, first because of growth conditions on the algebra [59], but also since the finitely-summable condition is very analogous to type II in the theory of factors. In very general cases, like the noncommutative space coming from foliations, one can however go from type III to type II by passing to the total space of the space of transverse metrics and then use the theory of hypoelliptic operators 82].

Another way to attack the problem of determining $D$ is to consider the larger algebra generated by $\mathcal{A}$ and $D$, write a-priori relations between $\mathcal{A}$ and $D$ and then look for irreducible representations that fall in the correct stable homotopy class. Ideally one should minimize the spectral action functional 45] in this homotopy class thus coming close to gravity. In practise one should use anything available and the example of the NC-space given by the quantum group $S U_{q}(2)$ shows that things can be quite subtle [192.

Once the spectral triple $(\mathcal{A}, \mathcal{H}, D)$ has been determined, the basic steps are the following, one should compute

- The dimension spectrum $\Sigma \subset \mathbb{C}$.

- The local index formula. 
- The inner fluctuations, scalar curvature, and spectral action.

4) Often a noncommutative space comes with a measure class, which in turn determines a time evolution $\sigma_{t}$, namely a 1-parameter family of automorphisms of the $C^{*}$-algebra $A=\overline{\mathcal{A}}$. In the type II situation one can apply the discussion of step 3 above and, in the finite dimensional case, use the operator $D$ to represent functionals in the measure class in the form

$$
\varphi(a)=f a|D|^{-p}, \quad \forall a \in \mathcal{A}
$$

where $f$ is the noncommutative integral i.e. the Dixmier trace and $p$ is the "dimension". In the general case one should expect to be in the type III situation in which the time evolution $\sigma_{t}$ is highly non-trivial. We shall see some examples, for instance in Section 22 Given the data $\left(\overline{\mathcal{A}}, \sigma_{t}\right)$ it is natural to regard it as a quantum statistical mechanical system, with $\overline{\mathcal{A}}$ as algebra of observables and $\sigma_{t}$ as time evolution. One can then look for equilibrium states for the system, for a given value $\beta$ of the thermodynamic parameter (inverse temperature).

If the algebra $\overline{\mathcal{A}}$ is concretely realized as an algebra of bounded operators on a Hilbert space $\mathcal{H}$, then one can consider the Hamiltonian $H$, namely the (unbounded) operator on $\mathcal{H}$ that is the infinitesimal generator of the time evolution. If the operator $\exp (-\beta H)$ is trace class, then one has equilibrium states for the system $\left(\overline{\mathcal{A}}, \sigma_{t}\right)$ written in the usual Gibbs form

$$
\varphi_{\beta}(a)=\frac{\operatorname{Tr}(a \exp (-\beta H))}{\operatorname{Tr}(\exp (-\beta H))},
$$

where $Z(\beta)=\operatorname{Tr}(\exp (-\beta H))$ is the partition function of the system. The notion of equilibrium state continues to make sense when $\exp (-\beta H)$ is not necessarily trace class, and is given by the more subtle notion of KMS (Kubo-Martin-Schwinger) states.

These are states on $\overline{\mathcal{A}}$, namely continuous functionals $\varphi: \overline{\mathcal{A}} \rightarrow \mathbb{C}$ with $\varphi(1)=1$ and $\varphi\left(a^{*} a\right) \geq$ 0 , satisfying the $\mathrm{KMS}_{\beta}$ condition that, for all $a, b \in \overline{\mathcal{A}}$ there exists a function $F_{a, b}(z)$ which is holomorphic on the strip $0<\Re(z)<i \beta$ continuous and bounded on the closed strip and such that, for all $t \in \mathbb{R}$,

$$
F_{a, b}(t)=\varphi\left(a \sigma_{t}(b)\right) \quad \text { and } \quad F_{a, b}(t+i \beta)=\varphi\left(\sigma_{t}(b) a\right) .
$$

KMS states at zero temperature can be defined as weak limits as $\beta \rightarrow \infty$ of $\mathrm{KMS}_{\beta}$ states. One can construct using KMS states very refined invariants of noncommutative spaces. For a fixed $\beta$, the $\mathrm{KMS}_{\beta}$ states form a simplex, hence one can consider only the extremal $\mathrm{KMS}_{\beta}$ states $\mathcal{E}_{\beta}$, from which one recovers all the others by convex combinations. An extremal $\mathrm{KMS}_{\beta}$ state is always factorial and the type of the factor is an invariant of the state. The simplest situation is the type I. One can show under minimal hypothesis (66]) that extremal $\mathrm{KMS}_{\beta}$ states continue to survive when one lowers the temperature i.e. one increases $\beta$. Thus, in essence, when cooling down the system this tends to become more and more "classical" and in the 0-temperature limit $\mathcal{E}_{\beta}$ gives a good replacement of the notion of classical points for a noncommutative space. We shall see in Section 24 how, in examples related to arithmetic, the "classical points" described by the zero temperature KMS states of certain quantum statistical mechanical systems recover classical arithmetic varieties. The extremal KMS states at zero temperature, evaluated on suitable arithmetic elements in the noncommutative algebra, can be shown in significant cases to have an interesting Galois action, related to interesting questions in number theory ( $c f$. 27], 78], 74]). 


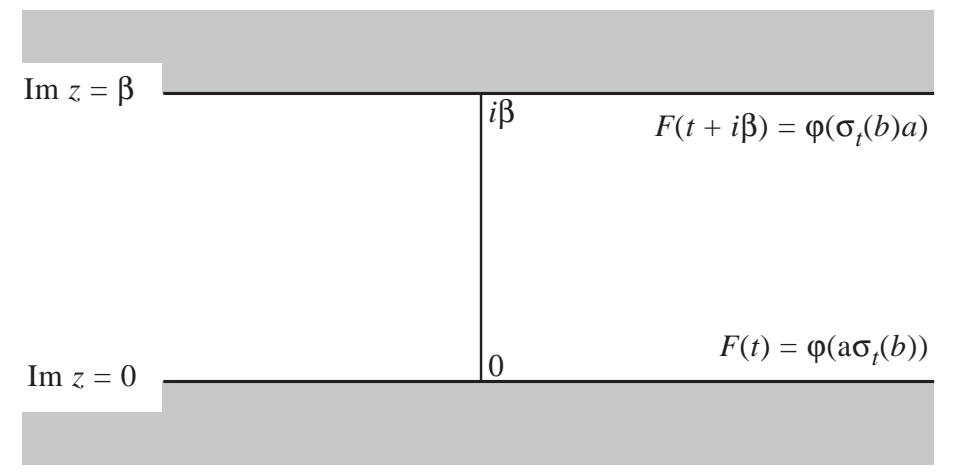

FiguRE 1. The KMS condition

In joint work with Consani, we showed in 66] how to define an analog in characteristic zero of the action of the Frobenius on the etale cohomology by a process involving the above thermodynamics. One key feature is that the analogue of the Frobenius is the "dual" of the above time evolution $\sigma_{t}$. The process involves cyclic homology and its three basic steps are $(\underline{66})$

- Cooling.

- Distillation.

- Dual action of $\mathbb{R}_{+}^{*}$ on the cyclic homology of the distilled space.

When applied to the simplest system (the Bost-Connes system of [27]) this yields a cohomological interpretation of the spectral realization of the zeros of the Riemann zeta function (64, 66]).

\section{Phase spaces of Microscopic Systems}

What can be regarded historically as the first example of a non-commutative space is the Heisenberg formulation of the observational Ritz-Rydberg law of spectrocopy. In fact, quantum mechanics showed that indeed the parameter space, or phase space of the mechanical system given by a single atom fails to be a manifold. It is important to convince oneself of this fact and to understand that this conclusion is indeed dictated by the experimental findings of spectroscopy.

At the beginning of the twentieth century a wealth of experimental data was being collected on the spectra of various chemical elements. These spectra obey experimentally discovered laws, the most notable being the Ritz-Rydberg combination principle. The principle can be stated as follows; spectral lines are indexed by pairs of labels. The statement of the principle then is that certain pairs of spectral lines, when expressed in terms of frequencies, do add up 


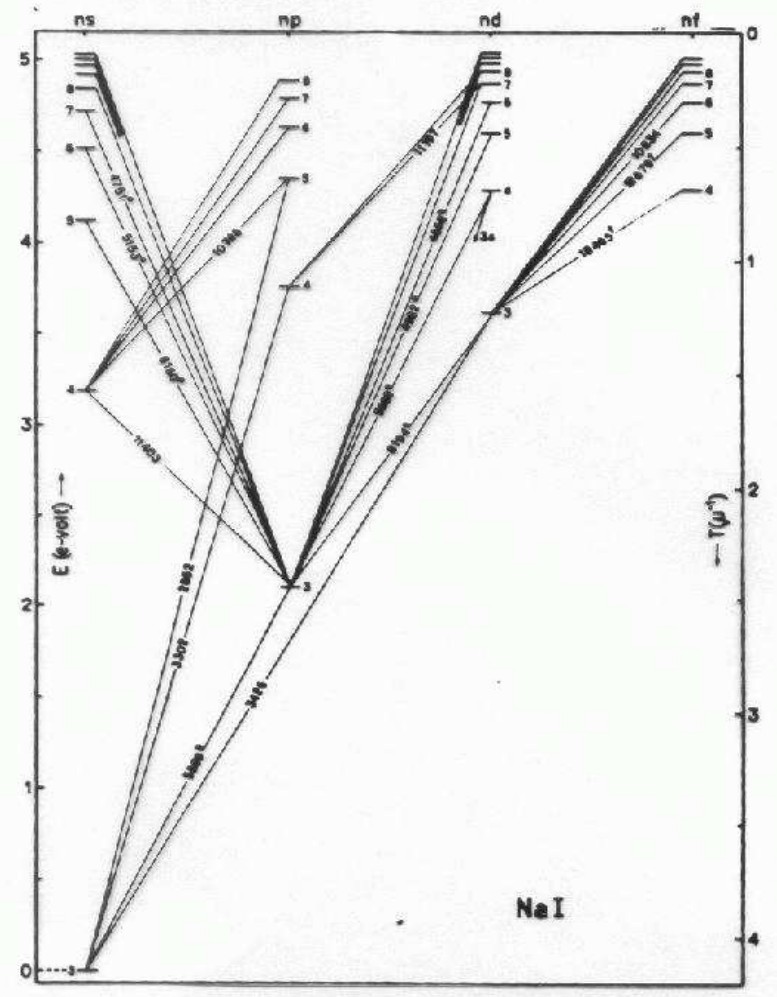

Figure 2. Spectral lines and the Ritz-Rydberg law.

to give another line in the spectrum. Moreover, this happens precisely when the labels are of the form $i, j$ and $j, k$.

In the seminal paper [116 of 1925, Heisenberg considers the classical prediction for the radiation emitted by a moving electron in a field, where the observable dipole moment can be computed, with the motion of the electron given in Fourier expansion. The classical model would predict (in his notation) frequencies distributed according to the law

$$
\nu(n, \alpha)=\alpha \nu(n)=\alpha \frac{1}{h} \frac{d W}{d n} .
$$

When comparing the frequencies obtained in this classical model with the data, Heisenberg noticed that the classical law (3.1) did not match the phenomenon observed.

The spectral rays provide a 'picture' of an atom: if atoms were classical systems, then the picture formed by the spectral lines would be (in our modern mathematical language) a group, which is what (3.1) predicts. That is, the classical model predicts that the observed frequencies should simply add, obeying a group law, or, in Heisenberg's notation, that

$$
\nu(n, \alpha)+\nu(n, \beta)=\nu(n, \alpha+\beta) .
$$

Correspondingly, observables would form the convolution algebra of a group. 
What the spectral lines were instead providing was the picture of a groupoid. Heisenberg realized that the classical law of (3.1) (3.2) would have to be replaced by the quantummechanical

$$
\nu(n, n-\alpha)=\frac{1}{h}(W(n)-W(n-\alpha)) .
$$

This replaces the group law with that of a groupoid, replacing the classical (3.2) by the quantum-mechanical

$$
\nu(n, n-\alpha)+\nu(n-\alpha, n-\alpha-\beta)=\nu(n, n-\alpha-\beta) .
$$

Similarly, the classical Fourier modes $\mathfrak{U}_{\alpha}(n) e^{i \omega(n) \alpha t}$ were replaced by $\mathfrak{U}(n, n-\alpha) e^{i \omega(n, n-\alpha) t}$. The analysis of the emission spectrum given by Heisenberg was in very good agreement with the Ritz-Rydberg law, or combination principle, for spectral lines in emission or absorption spectra.

In the same paper, Heisenberg also extends his redefinition of the multiplication law for the Fourier coefficients to coordinates and momenta, by introducing transition amplitudes that satisfy similar product rules. This is, in Born's words, "his most audacious step": in fact, it is precisely this step that brings non-commutative geometry on the scene.

It was Born who realized that what Heisenberg described in his paper corresponded to replacing classical coordinates with coordinates which no longer commute, but which obey the laws of matrix multiplication. In his own words reported in [197],

After having sent Heisenberg's paper to the Zeitschrift für Physik for publication, I began to ponder about his symbolic multiplication, and was soon so involved in it that I thought the whole day and could hardly sleep at night. For I felt there was something fundamental behind it ... And one morning ... I suddenly saw light: Heisenberg's symbolic multiplication was nothing but the matrix calculus.

Thus, spectral lines are parameterized by two indices $L_{\alpha \beta}$ satisfying a cocycle relation $L_{\alpha \beta}+$ $L_{\beta \gamma}=L_{\alpha \gamma}$, and a coboundary relation expresses each spectral line as a difference $L_{\alpha \beta}=$ $\nu_{\alpha}-\nu_{\beta}$. In other words, the Ritz-Rydberg law gives the groupoid law (3.4), or equivalently,

$$
(i, j) \bullet(j, k)=(i, k)
$$

and the convolution algebra of the group is replaced by observables satisfying the matrix product

$$
(A B)_{i k}=\sum_{j} A_{i j} B_{j k}
$$

for which in general commutativity is lost:

$$
A B \neq B A .
$$

The Hamiltonian $H$ is a matrix with the frequencies on the diagonal, and observables obey the evolution equation

$$
\frac{d}{d t} A=i[H, A] .
$$


Out of Heisenberg's paper and Born's interpretation of the same in terms of matrix calculus, emerged the statement of Heisenberg's uncertainty principle in the form of a commutation relation of matrices

$$
[P, Q]=\frac{h}{2 \pi i} I .
$$

The matrix calculus and the uncertainty principle were formulated in the subsequent paper of Born and Jordan [25], also published in 1925. This viewpoint on quantum mechanics was later somewhat obscured by the advent of the Schrödinger equation. The Schrödinger approach shifted the emphasis back to the more traditional technique of solving partial differential equations, while the more modern viewpoint of Heisenberg implied a much more serious change of paradigm, affecting our most basic understanding of the notion of space. Heisenberg's approach can be regarded as the historic origin of noncommutative geometry.

\section{Noncommutative QUotients}

A large source of examples of noncommutative spaces is given by quotients of equivalence relations. One starts by an ordinary commutative space $X$ (e.g. a smooth manifold or more generally a locally compact Hausdorff topological space). This can be described via its algebra of functions $C(X)$, and abelian $C^{*}$-algebra. Suppose then that we are interested in taking a quotient $Y=X / \sim$ of $X$ with respect to an equivalence relation. In general, one should not expect the quotient to be nice. Even when $X$ is a smooth manifold, the quotient $Y$ need not even be a Hausdorff space. In general, one would like to still be able to characterize $Y$ through its ring of functions. One usually defines $C(Y)$ to be functions on $X$ that are invariant under the equivalence relation ,

$$
C(Y)=\{f \in C(X): f(a)=f(b), \forall a \sim b\} .
$$

Clearly, for a "bad" equivalence relation one typically gets this way only constant functions $C(Y)=\mathbb{C}$.

There is a better way to associate to the quotient space $Y$ a ring of functions which is nontrivial for any equivalence relation. This requires dropping the commutativity requirement. One can then consider functions of two variables $f_{a b}$ defined on the graph of the equivalence relation, with a product which is no longer the commutative pointwise product, but the noncommutative convolution product dictated by the groupoid of the equivalence relation. In general the elements in the algebra of functions

$$
\text { "C } C(Y) "=\left\{\left(f_{a b}\right): a \sim b\right\}
$$

act as bounded operators on the Hilbert space $L^{2}$ of the equivalence class. This also guarantees the convergence in the operator norm of the convolution product

$$
\sum_{a \sim b \sim c} f_{a b} g_{b c} .
$$

We give a few examples to illustrate the difference between the traditional construction and the one of noncommutative geometry. 
Example 4.1. Consider the space $Y=\left\{x_{0}, x_{1}\right\}$ with the equivalence relation $x_{0} \sim x_{1}$. With the first point of view the algebra of functions on the quotient is $\mathbb{C}$, and in the second point of view it is $\mathcal{B}=M_{2}(\mathbb{C})$, that is

$$
\mathcal{B}=\left\{f=\left(\begin{array}{cc}
f_{a a} & f_{a b} \\
f_{b a} & f_{b b}
\end{array}\right)\right\}
$$

These two algebras are not the same, though in this case they are Morita equivalent.

Notice that, when one computes the spectrum of the algebra (4.3), it turns out that it is composed of only one point, so the two points $a$ and $b$ have been identified. This first trivial example represents the typical situation where the quotient space is "nice": the two constructions give Morita equivalent algebras. In this sense, Morita equivalent algebras are regarded as "the same" (or better isomorphic) spaces in non-commutative geometry.

Example 4.2. Consider the space $Y=[0,1] \times\{0,1\}$ with the equivalence relation $(x, 0) \sim$ $(x, 1)$ for $x \in(0,1)$. Then in the first viewpoint the algebra of functions is again given just by the constant functions $\mathbb{C}$, but in the second case we obtain

$$
\left\{f \in C([0,1]) \otimes M_{2}(\mathbb{C}): f(0) \text { and } f(1) \text { diagonal }\right\} .
$$

In this case, these algebras are not Morita equivalent. This can be seen by computing their $K$-theory. This means that the approach of non-commutative spaces produces something genuinely new, as soon as the quotient space ceases to be "nice" in the classical sense.

In general, the first kind of construction of functions on the quotient space is cohomological in nature: one seeks for functions satisfying certain equations or constraints. Usually there are very few solutions. The second approach, instead, typically produces a very large class of functions.

\section{Spaces of LeAVes of FOliations}

There is a very rich collection of examples of noncommutative spaces given by the leaf spaces of foliations. The connection thus obtained between noncommutative geometry and the geometric theory of foliations is very far reaching for instance through the role of Gelfand-Fuchs cohomology, of the Godbillon-Vey invariant and of the passage from type III to type II using the transverse frame bundle. It is this class of examples that triggered the initial development of cyclic cohomology ( $c f$. [126] section 4), of the local index formula in noncommutative geometry as well as the theory of characteristic classes for Hopf algebra actions.

The construction of the algebra associated to a foliation is a special case of the construction of section 4 but both the presence of holonomy and the case when the graph of the foliation is non-hausdorff require special care, so we shall recall the basic steps below.

Let $V$ be a smooth manifold and $T V$ its tangent bundle, so that for each $x \in V, T_{x} V$ is the tangent space of $V$ at $x$. A smooth subbundle $F$ of $T V$ is called integrable iff one of the following equivalent conditions is satisfied: 
a) Every $x \in V$ is contained in a submanifold $W$ of $V$ such that

$$
T_{y}(W)=F_{y} \quad \forall y \in W .
$$

b) Every $x \in V$ is in the domain $U \subset V$ of a submersion $p: U \rightarrow \mathbb{R}^{q}(q=\operatorname{codim} F)$ with

$$
F_{y}=\operatorname{Ker}\left(p_{*}\right)_{y} \quad \forall y \in U .
$$

c) $C^{\infty}(F)=\left\{X \in C^{\infty}(T V), X_{x} \in F_{x} \quad \forall x \in V\right\}$ is a Lie algebra.

d) The ideal $J(F)$ of smooth exterior differential forms which vanish on $F$ is stable by exterior differentiation.

Any 1-dimensional subbundle $F$ of $T V$ is integrable, but for $\operatorname{dim} F \geqq 2$ the condition is non trivial, for instance if $P \stackrel{p}{\rightarrow} B$ is a principal $H$-bundle (with compact structure group $H$ ) the bundle of horizontal vectors for a given connection is integrable iff this connection is flat.

A foliation of $V$ is given by an integrable subbundle $F$ of $T V$. The leaves of the foliation $(V, F)$ are the maximal connected submanifolds $L$ of $V$ with $T_{x}(L)=F_{x}, \forall x \in L$, and the partition of $V$ in leaves

$$
V=\cup L_{\alpha}, \quad \alpha \in X
$$

is characterized geometrically by its "local triviality": every point $x \in V$ has a neighborhood $U$ and a system of local coordinates $\left(x^{j}\right)_{j=1, \ldots, \operatorname{dim} V}$ called foliation charts, so that the partition of $U$ in connected components of leaves corresponds to the partition of

$$
\mathbb{R}^{\operatorname{dim} V}=\mathbb{R}^{\operatorname{dim} F} \times \mathbb{R}^{\operatorname{codim} F}
$$

in the parallel affine subspaces $\mathbb{R}^{\operatorname{dim} F} \times$ pt. These are the leaves of the restriction of $F$ and are called plaques.

The set $X=V / F$ of leaves of a foliation $(V, F)$ is in most cases a noncommutative space. In other words even though as a set it has the cardinality of the continuum it is in general not so at the effective level and it is in general impossible to construct a countable set of measurable functions on $V$ that form a complete set of invariants for the equivalence relation coming from the partition of $V$ in leaves $V=\cup L_{\alpha}$. Even in the simple cases in which the set $X=V / F$ of leaves is classical it helps to introduce the associated algebraic tools in order to get a feeling for their role in the general singular case.

To each foliation $(V, F)$ is canonically associated a $C^{*}$ algebra $C^{*}(V, F)$ which encodes the topology of the space of leaves. The construction is basically the same as the general one for quotient spaces of Section 4 , but there are interesting nuances coming from the presence of holonomy in the foliation context. To take this into account one first constructs a manifold $G, \operatorname{dim} G=\operatorname{dim} V+\operatorname{dim} F$, called the graph (or holonomy groupoid) of the foliation, which refines the equivalence relation coming from the partition of $V$ in leaves $V=\cup L_{\alpha}$. This construction is due to Thom, Pradines and Winkelnkemper, see 200].

An element $\gamma$ of $G$ is given by two points $x=s(\gamma), y=r(\gamma)$ of $V$ together with an equivalence class of smooth paths: $\gamma(t) \in V, t \in[0,1] ; \gamma(0)=x, \gamma(1)=y$, tangent to the bundle $F$ (i.e. with $\left.\dot{\gamma}(t) \in F_{\gamma(t)}, \forall t \in \mathbb{R}\right)$ up to the following equivalence: $\gamma_{1}$ and $\gamma_{2}$ are equivalent iff the holonomy of the path $\gamma_{2} \circ \gamma_{1}^{-1}$ at the point $x$ is the identity. The graph $G$ has an obvious composition law. For $\gamma, \gamma^{\prime} \in G$, the composition $\gamma \circ \gamma^{\prime}$ makes sense if $s(\gamma)=r\left(\gamma^{\prime}\right)$. If the leaf $L$ which contains both $x$ and $y$ has no holonomy, then the class in $G$ of the path $\gamma(t)$ only 
depends on the pair $(y, x)$. The condition of trivial holonomy is generic in the topological sense of dense $G_{\delta}$ 's. In general, if one fixes $x=s(\gamma)$, the map from $G_{x}=\{\gamma, s(\gamma)=x\}$ to the leaf $L$ through $x$, given by $\gamma \in G_{x} \mapsto y=r(\gamma)$, is the holonomy covering of $L$.

Both maps $r$ and $s$ from the manifold $G$ to $V$ are smooth submersions and the map $(r, s)$ to $V \times V$ is an immersion whose image in $V \times V$ is the (often singular) subset

$$
\{(y, x) \in V \times V: y \text { and } x \text { are on the same leaf }\} .
$$

In first approximation one can think of elements of $C^{*}(V, F)$ as continuous matrices $k(x, y)$, where $(x, y)$ varies in the set (5.1). We now describe this $C^{*}$ algebra in all details. We assume, for notational convenience, that the manifold $G$ is Hausdorff. Since this fails to be the case in very interesting examples, we also explain briefly how to remove this hypothesis.

The basic elements of $C^{*}(V, F)$ are smooth half densities $f \in C_{c}^{\infty}\left(G, \Omega^{1 / 2}\right)$ with compact support on $G$. The bundle $\Omega_{G}^{1 / 2}$ of half densities on $G$ is obtained as follows. One first defines a line bundle $\Omega_{V}^{1 / 2}$ on $\mathrm{V}$. For $x \in V$ one lets $\Omega_{x}^{1 / 2}$ be the one dimensional complex vector space of maps from the exterior power $\wedge^{k} F_{x}, k=\operatorname{dim} F$, to $\mathbb{C}$ such that

$$
\rho(\lambda v)=|\lambda|^{1 / 2} \rho(v) \quad \forall v \in \wedge^{k} F_{x}, \quad \forall \lambda \in \mathbb{R} .
$$

Then, for $\gamma \in G$, one can identify $\Omega_{\gamma}^{1 / 2}$ with the one dimensional complex vector space $\Omega_{y}^{1 / 2} \otimes \Omega_{x}^{1 / 2}$, where $\gamma: x \rightarrow y$. In other words

$$
\Omega_{G}^{1 / 2}=r^{*}\left(\Omega_{V}^{1 / 2}\right) \otimes s^{*}\left(\Omega_{V}^{1 / 2}\right) .
$$

Of course the bundle $\Omega_{V}^{1 / 2}$ is trivial on $V$, and we could choose once and for all a trivialisation $\nu$ turning elements of $C_{c}^{\infty}\left(G, \Omega^{1 / 2}\right)$ into functions. Let us however stress that the use of half densities makes all the construction completely canonical.

For $f, g \in C_{c}^{\infty}\left(G, \Omega^{1 / 2}\right)$, the convolution product $f * g$ is defined by the equality

$$
(f * g)(\gamma)=\int_{\gamma_{1} \circ \gamma_{2}=\gamma} f\left(\gamma_{1}\right) g\left(\gamma_{2}\right) .
$$

This makes sense because, for fixed $\gamma: x \rightarrow y$ and fixing $v_{x} \in \wedge^{k} F_{x}$ and $v_{y} \in \wedge^{k} F_{y}$, the product $f\left(\gamma_{1}\right) g\left(\gamma_{1}^{-1} \gamma\right)$ defines a 1-density on $G^{y}=\left\{\gamma_{1} \in G, r\left(\gamma_{1}\right)=y\right\}$, which is smooth with compact support (it vanishes if $\gamma_{1} \notin$ support $f$ ), and hence can be integrated over $G^{y}$ to give a scalar, namely $(f * g)(\gamma)$ evaluated on $v_{x}, v_{y}$.

The $*$ operation is defined by $f^{*}(\gamma)=\overline{f\left(\gamma^{-1}\right)}$, i.e. if $\gamma: x \rightarrow y$ and $v_{x} \in \wedge^{k} F_{x}, v_{y} \in \wedge^{k} F_{y}$ then $f^{*}(\gamma)$ evaluated on $v_{x}, v_{y}$ is equal to $\overline{f\left(\gamma^{-1}\right)}$ evaluated on $v_{y}, v_{x}$. We thus get a $*$ algebra $C_{c}^{\infty}\left(G, \Omega^{1 / 2}\right)$. For each leaf $L$ of $(V, F)$ one has a natural representation of this $*$ algebra on the $L^{2}$ space of the holonomy covering $\tilde{L}$ of $L$. Fixing a base point $x \in L$, one identifies $\tilde{L}$ with $G_{x}=\{\gamma, s(\gamma)=x\}$ and defines

$$
\left(\pi_{x}(f) \xi\right)(\gamma)=\int_{\gamma_{1} \circ \gamma_{2}=\gamma} f\left(\gamma_{1}\right) \xi\left(\gamma_{2}\right) \quad \forall \xi \in L^{2}\left(G_{x}\right),
$$

where $\xi$ is a square integrable half density on $G_{x}$. Given $\gamma: x \rightarrow y$ one has a natural isometry of $L^{2}\left(G_{x}\right)$ on $L^{2}\left(G_{y}\right)$ which transforms the representation $\pi_{x}$ in $\pi_{y}$. 
By definition $C^{*}(V, F)$ is the $C^{*}$ algebra completion of $C_{c}^{\infty}\left(G, \Omega^{1 / 2}\right)$ with the norm

$$
\|f\|=\sup _{x \in V}\left\|\pi_{x}(f)\right\|
$$

Note that $C^{*}(V, F)$ is always norm separable and admits a natural smooth subalgebra, namely the algebra $C_{c}^{\infty}(V, F)=C_{c}^{\infty}\left(G, \Omega^{1 / 2}\right)$ of smooth compactly supported half densities.

If the leaf $L$ has trivial holonomy then the representation $\pi_{x}, x \in L$, is irreducible. In general, its commutant is generated by the action of the (discrete) holonomy group $G_{x}^{x}$ in $L^{2}\left(G_{x}\right)$. If the foliation comes from a submersion $p: V \rightarrow B$, then its graph $G$ is $\{(x, y) \in$ $V \times V, p(x)=p(y)\}$ which is a submanifold of $V \times V$, and $C^{*}(V, F)$ is identical with the algebra of the continuous field of Hilbert spaces $L^{2}\left(p^{-1}\{x\}\right)_{x \in B}$. Thus (unless $\operatorname{dim} F=0$ ) it is isomorphic to the tensor product of $C_{0}(B)$ with the elementary $C^{*}$ algebra of compact operators. If the foliation comes from an action of a Lie group $H$ in such a way that the graph is identical with $V \times H$ (this is not always true even for flows) then $C^{*}(V, F)$ is identical with the reduced crossed product of $C_{0}(V)$ by $H$. Moreover the construction of $C^{*}(V, F)$ is local in the following sense.

If $V^{\prime} \subset V$ is an open set and $F^{\prime}$ is the restriction of $F$ to $V^{\prime}$, then the graph $G^{\prime}$ of $\left(V^{\prime}, F^{\prime}\right)$ is an open set in the graph $G$ of $(V, F)$, and the inclusion $C_{c}^{\infty}\left(G^{\prime}, \Omega^{1 / 2}\right) \subset C_{c}^{\infty}\left(G, \Omega^{1 / 2}\right)$ extends to an isometric $*$ homomorphism of $C^{*}\left(V^{\prime}, F^{\prime}\right)$ in $C^{*}(V, F)$. The proof is straightforward and also applies in the case of non Hausdorff graph.

Let us now briefly explain how the construction of the $C^{*}$ algebra $C^{*}(V, F)$ has to be done in the case when the graph of the foliation is not Hausdorff. This case is rather rare, since it never occurs if the foliation is real analytic. However, it does occur in cases which are topologically interesting for foliations, such as the Reeb foliation of the 3 sphere, which are constructed by patching together foliations of manifolds with boundaries $\left(V_{i}, F_{i}\right)$ where the boundary $\partial V_{i}$ is a leaf of $F_{i}$. In fact most of the constructions done in geometry to produce smooth foliations of given codimension on a given manifold give a non Hausdorff graph. The $C^{*}$-algebra $C^{*}(V, F)$ turns out in this case to be obtained as a fibered product of the $C^{*}\left(V_{i}, F_{i}\right)$.

In the general non-Hausdorff case the graph $G$ of $(V, F)$, being non Hausdorff may have only very few continuous fonctions with compact support. However, being a manifold, we can give a local chart $U \stackrel{\chi}{\rightarrow} \mathbb{R}^{\operatorname{dim} G}$. Take a smooth function $\varphi \in C_{c}^{\infty}\left(\mathbb{R}^{\operatorname{dim} G}\right), \operatorname{Supp} \varphi \subset \chi(U)$ and consider the function on $G$ equal to $\varphi \circ \chi$ on $U$ and to 0 outside $U$. If $G$ were Hausdorff, this would generate all of $C_{c}^{\infty}(G)$ by taking linear combinations, and in general we take this linear span as the definition of $C_{c}^{\infty}(G)$. Note that we do not get continuous functions, since there may well be a sequence $\gamma_{n} \in U$ with two limits, one in $\operatorname{Supp} \varphi \circ \chi$ one in the complement of $U$. The above definition of $C_{c}^{\infty}(G)$ obviously extends to get $C_{c}^{\infty}\left(G, \Omega^{1 / 2}\right)$ the space of smooth $\frac{1}{2}$ densities on $G$. One then shows that the convolution $\varphi_{1} * \varphi_{2}$ of $\varphi_{1}, \varphi_{2} \in C_{c}^{\infty}\left(G, \Omega^{1 / 2}\right)$ is in $C_{c}^{\infty}\left(G, \Omega^{1 / 2}\right)$.

Then we proceed exactly as in the Hausdorff case, and construct the representation $\pi_{x}$ of the * algebra $C_{c}^{\infty}\left(G, \Omega^{1 / 2}\right)$ in the Hilbert space $L^{2}\left(G_{x}\right)$. We note that though $G$ is not Hausdorff, each $G_{x}$ is Hausdorff, being the holonomy covering of the leaf through $x$.

For each $\varphi \in C_{c}^{\infty}\left(G, \Omega^{1 / 2}\right)$ and $x \in V, \pi_{x}(\varphi)$ is an ordinary smoothing operator, bounded in $L^{2}\left(G_{x}\right)$. 


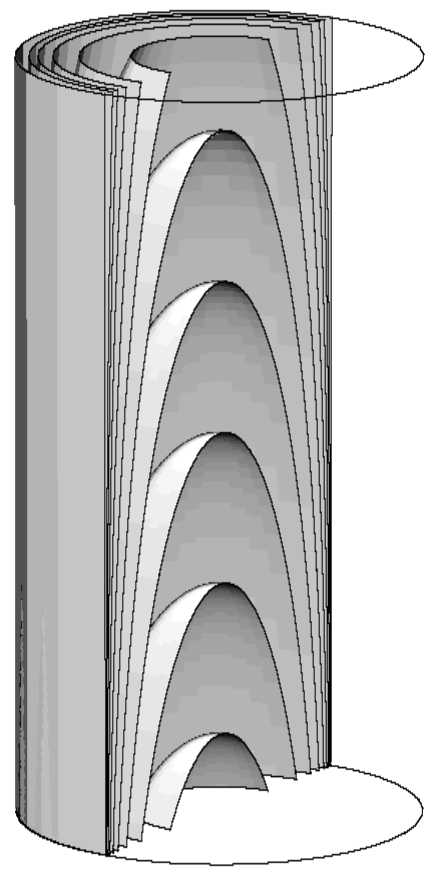

Figure 3. The Reeb foliation

Exactly as in the Hausdorff case $C^{*}(V, F)$ is defined as the $C^{*}$ completion of $C_{c}^{\infty}\left(G, \Omega^{1 / 2}\right)$ with norm $\sup _{x \in V}\left\|\pi_{x}(\varphi)\right\|$.

The obtained functor from foliations to $C^{*}$-algebras makes it possible first of all to translate from basic geometric properties to algebraic ones and the simplest examples of foliations already exhibit remarkable $C^{*}$-algebras. For instance the horocycle foliation of the unit sphere bundle of a Riemann surface of genus $>1$ gives a simple $C^{*}$-algebra without idempotent. The Kronecker foliation gives rise to the noncommutative torus, which we describe in more detail in Section 6 ,

In the type II situation i.e. in the presence of a holonomy invariant transverse measure $\Lambda$ the basic result of the theory is the longitudinal index theorem which computes the $L^{2}$-index of differential operators $D$ on the foliated manifold $(V, F)$ which are elliptic in the longitudinal direction (i.e. $D$ restrict to the leaves $L$ as elliptic operators $D_{L}$ ). One starts with a pair of smooth vector bundles $E_{1}, E_{2}$ on $V$ together with a differential operator $D$ on $V$ from sections of $E_{1}$ to sections of $E_{2}$ such that:

1) $D$ restricts to leaves, i.e. $(D \xi)_{x}$ only depends on the restriction of $\xi$ to a neighborhood of $x$ in the leaf of $x$ (i.e. $D$ only uses partial differentiation in the leaf direction).

2) $D$ is elliptic when restricted to any leaf.

Theorem 5.1. 52

a) There exists a Borel transversal $B$ (resp. $\left.B^{\prime}\right)$ such that the bundle $\left(\ell^{2}(L \cap B)\right)_{L \in V / F}$ is measurably isomorphic to the bundle $\left(\operatorname{Ker} D_{L}\right)_{L \in V / F}\left(\right.$ resp. to $\left.\left(\operatorname{Ker} D_{L}^{*}\right)_{L \in V / F}\right)$. 
b) The scalar $\Lambda(B)<\infty$ is independent of the choice of $B$ and noted $\operatorname{dim}_{\Lambda}(\operatorname{Ker}(D))$.

c) $\operatorname{dim}_{\Lambda}(\operatorname{Ker}(D))-\operatorname{dim}_{\Lambda}\left(\operatorname{Ker}\left(D^{*}\right)\right)=\varepsilon\left\langle\operatorname{ch} \sigma_{D} \operatorname{Td}\left(F_{\mathbb{C}}\right),[C]\right\rangle$

$\left(\varepsilon=(-1) \frac{k(k+1)}{2}, k=\operatorname{dim} F, \operatorname{Td}\left(F_{\mathbb{C}}\right)=\right.$ Todd genus, $\sigma_{D}=$ symbol of $\left.D\right)$.

Here $[C] \in H_{k}(V, \mathbb{C})$ is the homology class of the Ruelle-Sullivan current, a closed de-Rham current of dimension $k=\operatorname{dim} F$ which encodes the transverse measure $\Lambda$ by integration of a $k$-dimensional differential form $\omega$ on $V$ along the plaques of foliation charts.

In particular the Betti numbers $\beta_{j}$ of a measured foliation were defined in 52 and give the $L^{2}$-dimension of the space of $L^{2}$-harmonic forms along the leaves, more precisely one has the following result.

a) For each $j=0,1,2, \ldots, \operatorname{dim} F$, there exists a Borel transversal $B_{j}$ such that the bundle $\left(H^{j}(L, \mathbb{C})\right)_{L \in V / F}$ of $j$-th square integrable harmonic forms on $L$ is measurably isomorphic to $\left(\ell^{2}(L \cap B)\right)_{L \in V / F}$.

b) The scalar $\beta_{j}=\Lambda\left(B_{j}\right)$ is finite, independent of the choice of $B_{j}$, of the choice of the Euclidean structure on $F$.

c) One has $\Sigma(-1)^{j} \beta_{j}=\chi(F, \Lambda)$.

Here the Euler characteristic is simply given by the pairing of the Ruelle-Sullivan current with the Euler class $e(F)$ of the oriented bundle $F$ on $V$.

Extending ideas of Cheeger and Gromov 47 in the case of discrete groups, D. Gaboriau has shown in a remarkable recent work ( $c f$. [104], [105]) that the Betti numbers $\beta_{j}(F, \Lambda)$ of a foliation with contractible leaves are invariants of the measured equivalence relation $\mathcal{R}=\{(x, y) \mid y \in \operatorname{leaf}(x)\}$.

In the general case one cannot expect to have a holonomy invariant transverse measure and in fact the simplest foliations are of type III from the measure theoretic point of view. Obtaining an analogue in general of Theorem [5.1 was the basic motivation for the construction of the assembly map (the second step of section 2). Let us now briefly state the longitudinal index theorem.

Let $D$ be as above an elliptic differential operator along the leaves of the foliation $(V, F)$. Since $D$ is elliptic it has an inverse modulo $C^{*}(V, F)$ hence it gives an element $\operatorname{Ind}_{a}(D)$ of $K_{0}\left(C^{*}(V, F)\right)$ which is the analytic index of $D$. The topological index is obtained as follows. Let $i$ be an auxiliary imbedding of the manifold $V$ in $\mathbb{R}^{2 n}$. Let $N$ be the total space of the normal bundle to the leaves: $N_{x}=\left(i_{*}\left(F_{x}\right)\right)^{\perp} \subset \mathbb{R}^{2 n}$. Let us foliate $\tilde{V}=V \times \mathbb{R}^{2 n}$ by $\tilde{F}, \tilde{F}_{(x, t)}=F_{x} \times\{0\}$, so that the leaves of $(\tilde{V}, \tilde{F})$ are just $\tilde{L}=L \times\{t\}$, where $L$ is a leaf of $(V, F)$ and $t \in \mathbb{R}^{2 n}$. The map $(x, \xi) \rightarrow(x, i(x)+\xi)$ turns an open neighborhood of the 0 -section in $N$ into an open transversal $T$ of the foliation $(\tilde{V}, \tilde{F})$. For a suitable open neighborhood $\Omega$ of $T$ in $\tilde{V}$, the $C^{*}$-algebra $C^{*}(\Omega, \tilde{F})$ of the restriction of $\tilde{F}$ to $\Omega$ is (Morita) equivalent to $C_{0}(T)$, hence the inclusion $C^{*}(\Omega, \tilde{F}) \subset C^{*}(\tilde{V}, \tilde{F})$ yields a $K$-theory map: $K^{0}(N) \rightarrow K_{0}\left(C^{*}(\tilde{V}, \tilde{F})\right)$. Since $C^{*}(\tilde{V}, \tilde{F})=C^{*}(V, F) \otimes C_{0}\left(\mathbb{R}^{2 n}\right)$, one has, by Bott periodicity, the equality $K_{0}\left(C^{*}(\tilde{V}, \tilde{F})\right)=K_{0}\left(C^{*}(V, F)\right)$.

Using the Thom isomorphism, $K^{0}\left(F^{*}\right)$ is identified with $K^{0}(N)$ so that one gets by the above construction the topological index:

$$
\operatorname{Ind}_{t}: K^{0}\left(F^{*}\right) \rightarrow K_{0}\left(C^{*}(V, F)\right) .
$$


The longitudinal index theorem [89] is the equality

$$
\operatorname{Ind}_{a}(D)=\operatorname{Ind}_{t}\left(\left[\sigma_{D}\right]\right),
$$

where $\sigma_{D}$ is the longitudinal symbol of $D$ and $\left[\sigma_{D}\right]$ is its class in $K^{0}\left(F^{*}\right)$.

Since the group $K_{0}\left(C^{*}(V, F)\right)$ is still fairly hard to compute one needs computable invariants of its elements and this is where cyclic cohomology enters the scene. In fact its early development was already fully completed in 1981 for that precise goal ( $c f$. [126]). The role of the trace on $C^{*}(V, F)$ associated to the transverse measure $\Lambda$ is now played by cyclic cocycles on a dense subalgebra of $C^{*}(V, F)$. The hard analytic problem is to show that these cocycles have enough semi-continuity properties to define invariants of $K_{0}\left(C^{*}(V, F)\right)$. This was achieved for some of them in [56] and makes it possible to formulate corollaries whose statements are independent of the general theory, such as

Theorem 5.2. 56 Let $M$ be a compact oriented manifold and assume that the $\hat{A}$-genus $\hat{A}(M)$ is non-zero (since $M$ is not assumed to be a spin manifold $\hat{A}(M)$ need not be an integer). Let then $F$ be an integrable Spin sub-bundle of TM. There exists no metric on $F$ for which the scalar curvature (of the leaves) is strictly positive $(\geq \varepsilon>0)$ on $M$.

There is a very rich interplay between the theory of foliations and their characteristic classes and operator algebras even at the purely measure theoretic level i.e. the classification of factors.

In a remarkable series of papers (see 119] for references), J. Heitsch and S. Hurder have analyzed the interplay between the vanishing of the Godbillon-Vey invariant of a compact foliated manifold $(V, F)$ and the type of the von Neumann algebra of the foliation. Their work culminates in the following beautiful result of S. Hurder (119]). If the von Neumann algebra is semi-finite, then the Godbillon-Vey invariant vanishes. We have shown, in fact, that cyclic cohomology yields a stronger result, proving that, if $\mathrm{GV} \neq 0$, then the central decomposition of $M$ contains necessarily factors $M$, whose virtual modular spectrum is of finite covolume in $\mathbb{R}_{+}^{*}$.

Theorem 5.3. 56] Let $(V, F)$ be an oriented, transversally oriented, compact, foliated manifold, $(\operatorname{codim} F=1)$. Let $M$ be the associated von Neumann algebra, and $\operatorname{Mod}(M)$ be its flow of weights. Then, if the Godbillon-Vey class of $(V, F)$ is different from 0 , there exists an invariant probability measure for the flow $\operatorname{Mod}(M)$.

One actually constructs an invariant measure for the flow $\operatorname{Mod}(M)$, exploiting the following remarkable property of the natural cyclic 1-cocycle $\tau$ on the algebra $\mathcal{A}$ of the transverse 1-jet bundle for the foliation. When viewed as a linear map $\delta$ from $\mathcal{A}$ to its dual, $\delta$ is an unbounded derivation, which is closable, and whose domain extends to the center $Z$ of the von-Neumann algebra generated by $\mathcal{A}$. Moreover, $\delta$ vanishes on this center, whose elements $h \in Z$ can then be used to obtain new cyclic cocycles $\tau_{h}$ on $\mathcal{A}$. The pairing

$$
L(h)=\left\langle\tau_{h}, \mu(x)\right\rangle
$$

with the K-theory classes $\mu(x)$ obtained from the assembly map $\mu$, which we had constructed with P. Baum [15, then gives a measure on $Z$, whose invariance under the flow of weights follows from the discreteness of the K-group. To show that it is non-zero, one uses an index 
formula that evaluates the cyclic cocycles, associated as above to the Gelfand-Fuchs classes, on the range of the assembly map $\mu$.

The central question in the analysis of the noncommutative leaf space of a foliation is step 3) (of section 2), namely the metric aspect which entails in particular constructing a spectral triple describing the transverse geometry. The reason why the problem is really difficult is that it essentially amounts to doing "metric" geometry on manifolds in a way which is "background independent" to use the terminology of physicists i.e. which is invariant under diffeomorphisms rather than covariant as in traditional Riemannian geometry. Indeed the transverse space of a foliation is a manifold endowed with the action of a large pseudo group of partial diffeomorphisms implementing the holonomy. Thus in particular no invariant metric exists in the general case and the situation is very similar to trying to develop gravity without making use of any particular "background" metric that automatically destroys the invariance under the action of diffeomeorphisms ( $c f .[86]$ ). Using the theory of hypoelliptic differential operators and the basic technique of reduction from type III to type II, a general construction of a spectral triple was done by Connes-Moscovici in [81]. The remaining problem of the computation of the local index formula in cyclic cohomology was solved in [82] and led in particular to the discovery of new symmetries given by an action of a Hopf algebra which only depends upon the transverse dimension of the foliation.

This also led to the development of the noncommutative analogue of the Chern-Weil theory of characteristic classes [83] in the general context of Hopf algebra actions on noncommutative spaces and cyclic cohomology, a subject which is undergoing rapid progress, in particular thanks to the recent works of M. Khalkhali and collaborators [127], 128], [129], 113].

\section{The noncommutative TORI}

This is perhaps considered as the prototype example of a noncommutative space, since it illustrates very clearly the properties and structures of noncommutative geometries. Noncommutative tori played a key role in the early developments of the theory in the 1980's ( $c f$. [57]), giving rise to noncommutative analogues of vector bundles, connections, curvature, etc.

One can regard noncommutative tori as a special case of noncommutative spaces arising from foliations. In this case, one considers certain vector fields on the ordinary 2-dimensional real torus $T^{2}=\mathbb{R}^{2} / \mathbb{Z}^{2}$. In fact, one considers on $T^{2}$ the Kronecker foliation $d x=\theta d y$, where $\theta$ is a given real number. We are especially interested in the case where $\theta$ is irrational. That is, we consider the space of solutions of the differential equation,

$$
d x=\theta d y, \quad \forall x, y \in \mathbb{R} / \mathbb{Z}
$$

for $\theta \in] 0,1[$ is a fixed irrational number. In other words, we are considering the space of leaves of the Kronecker foliation on the torus ( $c f$. Figure 4).

We can choose a transversal $T$ to the foliation, given by

$$
T=\{y=0\}, \quad T \cong S^{1} \cong \mathbb{R} / \mathbb{Z} .
$$

Two points of the transversal which differ by an integer multiple of $\theta$ give rise to the same leaf. We want to describe the further quotient

$$
S^{1} / \theta \mathbb{Z}
$$




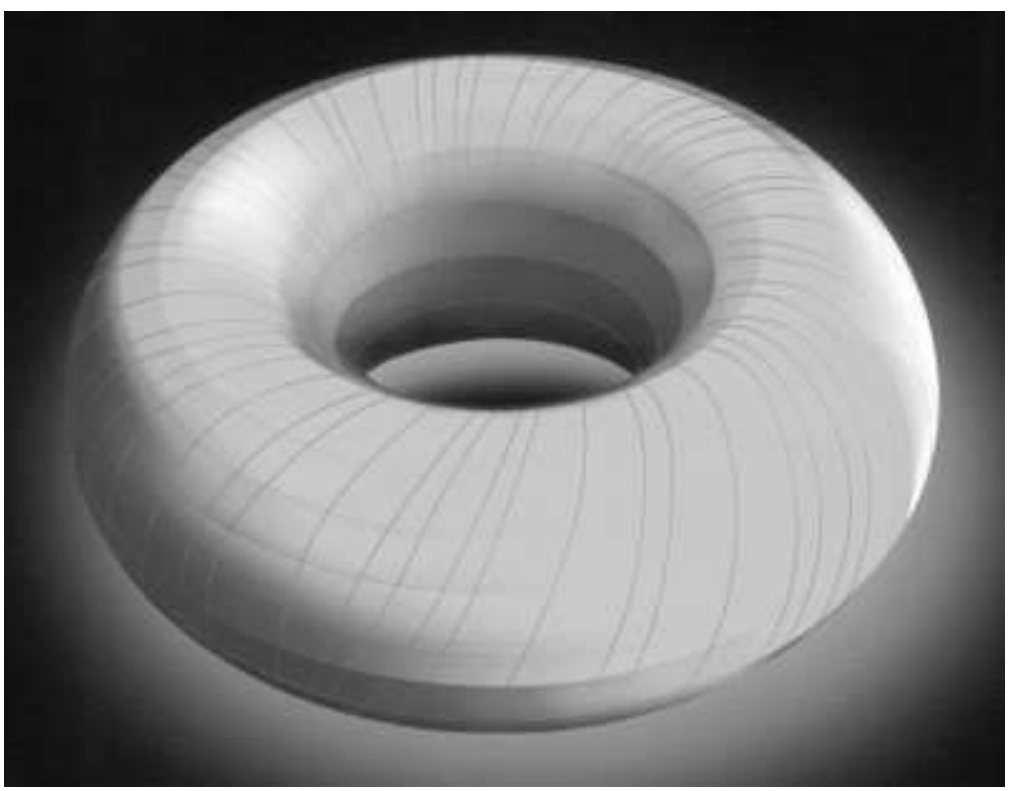

FiguRE 4. The Kronecker foliation and the noncommutative torus

by the equivalence relation which identifies any two points on the orbits of the irrational rotation

$$
R_{\theta} x=x+\theta \bmod 1 .
$$

We can regard the circle $S^{1}$ and the quotient space (6.2) at various levels of regularity (smooth, topological, measurable). This corresponds to different algebras of functions on the circle,

$$
C^{\infty}\left(S^{1}\right) \subset C\left(S^{1}\right) \subset L^{\infty}\left(S^{1}\right) .
$$

When passing to the quotient (6.2), if we just consider invariant functions we obtain a very poor algebra of functions, since, even at the measurable level, we would only have constant functions. If instead we consider the noncommutative algebra of functions obtained by the general recipe of "noncommutative quotients" (functions on the graph of the equivalence relation with the convolution product), we obtain a very interesting and highly non-trivial algebra of functions describing the space of leaves of the foliation. This is given (in the topological category) by the "irrational rotation algebra", i.e. the $C^{*}$-algebra

$$
\mathcal{A}_{\theta}:=\left\{\left(a_{i j}\right) \quad i, j \in T \text { in the same leaf }\right\} .
$$

Namely, elements in the algebra $\mathcal{A}_{\theta}$ associated to the transversal $T \simeq S^{1}$ are just matrices $\left(a_{i j}\right)$ where the indices are arbitrary pairs of elements $i, j$ of $S^{1}$ belonging to the same leaf. The algebraic rules are the same as for ordinary matrices. In the above situation, since the equivalence is given by a group action, the construction coincides with the crossed product. For instance, in the topological category, $\mathcal{A}_{\theta}$ is identified with the crossed product $C^{*}$-algebra

$$
\mathcal{A}_{\theta}=C\left(S^{1}\right) \rtimes_{R_{\theta}} \mathbb{Z}
$$


The algebra (6.5) has two natural generators:

$$
U= \begin{cases}1 & n=1 \\ 0 & \text { otherwise }\end{cases}
$$

and

$$
V= \begin{cases}e^{2 \pi i a} & n=0 \\ 0 & \text { otherwise }\end{cases}
$$

In fact, an element $b=\left(a_{i j}\right)$ of $\mathcal{A}_{\theta}$ can be written as power series

$$
b=\sum_{n \in \mathbb{Z}} b_{n} U^{n},
$$

where each $b_{n}$ is an element of the algebra (6.4), with the multiplication rule given by

$$
U h U^{-1}=h \circ R_{\theta}^{-1} .
$$

The algebra (6.4) is generated by the function $V$ on $S^{1}$,

$$
V(\alpha)=\exp (2 \pi i \alpha) \quad \forall \alpha \in S^{1}
$$

and it follows that $\mathcal{A}_{\theta}$ is generated by two unitaries $(U, V)$ with presentation given by the relation

$$
V U=\lambda U V, \quad \text { with } \quad \lambda=\exp (2 \pi i \theta) .
$$

If we work in the smooth category, then a generic element $b$ in (6.4) is given by a power series

$$
b=\sum_{\mathbb{Z}^{2}} b_{n m} U^{n} V^{m} \in \mathcal{S}\left(\mathbb{Z}^{2}\right)
$$

where $\mathcal{S}\left(\mathbb{Z}^{2}\right)$ is the Schwartz space of sequences of rapid decay on $\mathbb{Z}^{2}$. We refer to the algebra of smooth functions (6.13) as $\mathcal{C}^{\infty}\left(\mathbb{T}_{\theta}^{2}\right)$, where we think of $\mathbb{T}_{\theta}^{2}$ as the (smooth) non-commutative torus.

Notice that in the definition (6.5) it is not necessary to restrict to the condition that $i, j$ lie on the transversal $T$. It is possible to also form an algebra

$$
\mathcal{B}_{\theta}=\left\{\left(a_{i j}\right) \quad i, j \in T^{2} \text { in the same leaf }\right\},
$$

where now the parameter of integration is no longer discrete. This ought to correspond to the same non-commutative space, and in fact the algebras are related by

$$
\mathcal{B}_{\theta}=\mathcal{A}_{\theta} \otimes \mathcal{K},
$$

where $\mathcal{K}$ is the algebra of all compact operators.

The tangent space to the ordinary torus $T^{2}$ is spanned by the tangent directions $\frac{\partial}{\partial x}$ and $\frac{\partial}{\partial y}$. By choosing coordinates $U, V$, with $U=e^{2 \pi i x}$ and $V=e^{2 \pi i y}$, the tangent vectors are given by $\frac{\partial}{\partial x}=2 \pi i U \frac{\partial}{\partial U}$ and $\frac{\partial}{\partial y}=2 \pi i V \frac{\partial}{\partial V}$. These have analogs in terms of derivations of the algebra of the non-commutative torus. The two commuting vector fields which span the tangent space for an ordinary (commutative) 2-torus correspond algebraically to two commuting derivations of the algebra of smooth functions. 
These derivations continue to make sense when we replace the generators $U$ and $V$ of $C^{\infty}\left(\mathbb{T}^{2}\right)$ by the generators of the algebra $\mathcal{C}^{\infty}\left(\mathbb{T}_{\theta}^{2}\right)$, which no longer commute, as shown in (6.12). The derivations are still given by the same formulas as in the commutative case,

$$
\delta_{1}=2 \pi i U \frac{\partial}{\partial U} \quad \delta_{2}=2 \pi i V \frac{\partial}{\partial V}
$$

so that $\delta_{1}\left(\sum b_{n m} U^{n} V^{m}\right)=2 \pi i \sum n b_{n m} U^{n} V^{m}$, and similarly for $\delta_{2}$.

The operators (6.15) are commuting derivations of the algebra $\mathcal{C}^{\infty}\left(\mathbb{T}_{\theta}^{2}\right)$.

In fact, it is straightforward to verify that $\delta_{1}$ and $\delta_{2}$ satisfy

$$
\delta_{1} \delta_{2}=\delta_{2} \delta_{1}
$$

and

$$
\delta_{j}\left(b b^{\prime}\right)=\delta_{j}(b) b^{\prime}+b \delta_{j}\left(b^{\prime}\right) \quad \forall b, b^{\prime} \in \mathcal{A}_{\theta}
$$

Just as in the classical case of a (commutative) manifold, what ensures that the derivations considered are enough to span the whole tangent space is the condition of ellipticity for the Laplacian

$$
\Delta=\delta_{1}^{2}+\delta_{2}^{2} .
$$

In Fourier modes the Laplacian is of the form $n^{2}+m^{2}$, hence $\Delta^{-1}$ is a compact operator.

The geometry of the Kronecker foliation is closely related to the structure of the algebra. In fact, a choice of a closed transversal $T$ of the foliation corresponds canonically to a finite projective module over the algebra $\mathcal{A}_{\theta}$.

In fact, the main result on finite projective module over the non-commutative tori $\mathbb{T}_{\theta}^{2}$ is the following classification, which is obtained by combining the results of [167], [57, [175].

Theorem 6.1. Finite projective modules over $\mathcal{A}_{\theta}$ are classified up to isomorphism by a pair of integers $(p, q)$ such that $p+q \theta \geq 0$. For a choice of such pair, the corresponding module $\mathcal{H}_{p, q}^{\theta}$ is obtained from the transversal $T_{p, q}$ given by the closed geodesic of the torus $T^{2}$ specified by $(p, q)$, via the following construction. Elements of the module associated to the transversal $T_{p, q}$ are rectangular matrices, $\left(\xi_{i, j}\right)$ with $(i, j) \in T \times S^{1}$, and with $i$ and $j$ belonging to the same leaf. The right action of $\left(a_{i, j}\right) \in \mathcal{A}_{\theta}$ is by matrix multiplication.

For instance, from the transversal $x=0$ one obtains the following right module over $\mathcal{A}_{\theta}$. The underlying linear space is the usual Schwartz space

$$
\mathcal{S}(\mathbb{R})=\{\xi: \xi(s) \in \mathbb{C}, \quad \forall s \in \mathbb{R}\}
$$

of complex valued smooth functions on $\mathbb{R}$, all of whose derivatives are of rapid decay. The right module structure is given by the action of the generators $U, V$

$$
(\xi U)(s)=\xi(s+\theta) \quad(\xi V)(s)=e^{2 \pi i s} \xi(s) \quad \forall s \in \mathbb{R} .
$$

One of course checks that the relation (6.12) is satisfied, and that, as a right module over $\mathcal{A}_{\theta}$, the space $\mathcal{S}(\mathbb{R})$ is finitely generated and projective (i.e. it complements to a free module).

Finitely generated projective modules play an important role in noncommutative geometry, as they replace vector bundles in the commutative setting. In fact, in ordinary commutative geometry, one can equivalently describe vector bundles through their sections, which in turn form a finite projective module over the algebra of smooth functions. The notion of finite 
projective module continues to make sense in the non-commutative setting, and provides this way a good notion of "non-commutative vector bundles".

Suppose given a vector bundle $E$, described algebraically through its space of smooth sections $C^{\infty}(X, E)$. One can compute the dimension of $E$ by computing the trace of the identity endomorphism. In terms of the space of smooth sections, hence of finite projective modules, it is possible to recover the dimension of the vector bundle as a limit

$$
\operatorname{dim}_{\mathcal{A}}(\mathcal{E})=\lim _{N \rightarrow \infty} \frac{1}{N}(\# \text { Generators of } \underbrace{\mathcal{E} \oplus \cdots \oplus \mathcal{E}}_{N \text { times }}) .
$$

This method applies to the noncommutative setting. In the case of noncommutative tori, one finds that the Schwartz space $\mathcal{S}(\mathbb{R})$ has dimension the real number

$$
\operatorname{dim}_{\mathcal{B}}(\mathcal{S})=\theta .
$$

One similarly finds values $p+q \theta$ for the more general case of Theorem 6.1

The appearance of a real values dimension is related to the density of transversals in the leaves, that is, the limit of

$$
\frac{\# B_{R} \cap S}{\text { size of } B_{R}}
$$

for a ball $B_{R}$ of radius $R$ in the leaf. In this sense, the dimension $\theta$ of the Schwartz space measure the relative densities of the two transversals $S=\{x=0\}$ and $T=\{y=0\}$.

In general, the appearance of non integral dimension is a basic feature of von Neumann algebras of type II. The dimension of a vector bundle is the only invariant that remains when one looks from the measure theoretic point of view, using the algebra of measurable functions $L^{\infty}\left(S^{1}\right)$ in (6.4). The von Neumann algebra which describes the quotient space from the measure theoretic point of view is the crossed product

$$
R=L^{\infty}\left(S^{1}\right) \rtimes_{R_{\theta}} \mathbb{Z} .
$$

This is the well known hyperfinite factor of type $\mathrm{II}_{1}$. In particular the classification of finite projective modules $\mathcal{E}$ over $R$ is given by a positive real number, the Murray and von Neumann dimension

$$
\operatorname{dim}_{R}(\mathcal{E}) \in \mathbb{R}_{+} .
$$

The simplest way to describe the phenomenon of Morita equivalence for non-commutative tori is in terms of the Kronecker foliation, where it corresponds to reparameterizing the leaves space in terms of a different closed transversal. Thus, Morita equivalence of the algebras $\mathcal{A}_{\theta}$ and $\mathcal{A}_{\theta^{\prime}}$ for $\theta$ and $\theta^{\prime}$ in the same $\operatorname{PGL}(2, \mathbb{Z})$ orbit becomes simply a statement that the leaf-space of the original foliation is independent of the transversal used to parameterize it. For instance, Morita equivalence between $\mathcal{A}_{\theta}$ and $\mathcal{A}_{-1 / \theta}$ corresponds to changing the parameterization of the space of leaves from the transversal $T=\{y=0\}$ to the transversal $S=\{x=0\}$.

More generally, an explicit construction of bimodules $\mathcal{M}_{\theta, \theta^{\prime}}$ was obtained in [57]. These are given by the Schwartz space $\mathcal{S}(\mathbb{R} \times \mathbb{Z} / c)$, with the right action of $\mathcal{A}_{\theta}$ given by

$$
U f(x, u)=f\left(x-\frac{c \theta+d}{c}, u-1\right)
$$




$$
V f(x, u)=\exp (2 \pi i(x-u d / c)) f(x, u)
$$

and the left action of $\mathcal{A}_{\theta^{\prime}}$

$$
\begin{gathered}
U^{\prime} f(x, u)=f\left(x-\frac{1}{c}, u-a\right) \\
V^{\prime} f(x, u)=\exp \left(2 \pi i\left(\frac{x}{c \theta+d}-\frac{u}{c}\right)\right) f(x, u) .
\end{gathered}
$$

The bimodule $\mathcal{M}_{\theta, \theta^{\prime}}$ realizes the Morita equivalences between $\mathcal{A}_{\theta}$ and $\mathcal{A}_{\theta^{\prime}}$ for

$$
\theta^{\prime}=\frac{a \theta+b}{c \theta+d}=g \theta
$$

with $g \in \mathrm{PGL}(2, \mathbb{Z}), c f$. [57], [174].

\section{Duals of Discrete Groups}

Noncommutative geometry provides naturally a generalization of Pontrjagin duality for discrete groups. While the Pontrjagin dual $\hat{\Gamma}$ of a finitely generated discrete abelian group is a compact abelian group, the dual of a more general finitely generated discrete group is a noncommutative space.

To see this, recall that the usual Pontrjagin duality assigns to a finitely generated discrete abelian group $\Gamma$ its group of characters $\hat{\Gamma}=\operatorname{Hom}(\Gamma, U(1))$. The duality is given by Fourier transform $e^{i\langle k, \gamma\rangle}$, for $\gamma \in \Gamma$ and $k \in \hat{\Gamma}$.

In particular, Fourier transform gives an identification between the algebra of functions on $\hat{\Gamma}$ and the (reduced) $C^{*}$-algebra of the group $\Gamma$,

$$
C(\hat{\Gamma}) \cong C_{r}^{*}(\Gamma)
$$

where the reduced $C^{*}$-algebra $C_{r}^{*}(\Gamma)$ is the $C^{*}$-algebra generated by $\Gamma$ in the regular representation on $\ell^{2}(\Gamma)$.

When $\Gamma$ is non-abelian Pontrjagin duality no longer applies in the classical sense. However, the left hand side of (7.1) still makes sense and it behaves "like" the algebra of functions on the dual group. One can then say that, for a non-abelian group, the Pontrjagin dual $\hat{\Gamma}$ still exists as a noncommutative space whose algebra of coordinates is the $C^{*}$-algebra $C_{r}^{*}(\Gamma)$.

As an example that illustrates this general philosophy we give a different version of Example 4.2 .

Example 7.1. The algebra (4.4) of Example 4.2 is the group ring of the dihedral group $\mathbb{Z} \rtimes \mathbb{Z} / 2 \cong \mathbb{Z} / 2 * \mathbb{Z} / 2$.

In fact, first notice that to a representation of the group $\mathbb{Z} / 2 * \mathbb{Z} / 2$ (free product of two copies of the group with two elements) is the same thing as a pair of subspaces in the Hilbert space, $E, F \subset \mathcal{H}$. The corresponding operators are $U=I-2 P_{E}, V=I-2 P_{V}$, with $P_{E}, P_{V}$ the projections. The operators $U, V$ represent reflections, since $U=U^{*}, U^{2}=I$, $V=V^{*}, V^{2}=I$. The group $\Gamma=\mathbb{Z} / 2 * \mathbb{Z} / 2$ realized as words in the generators $U$ and $V$ can equivalently be described as the semi-direct product $\Gamma=\mathbb{Z} \rtimes \mathbb{Z} / 2$, by setting $X=U V$, with the action $U X U^{-1}=X^{-1}$. The regular representation of $\Gamma$ is analyzed using Mackey's theory for semi-direct products. One considers first representations of the normal subgroup, 
and then orbits of the action of $\mathbb{Z} / 2$. The irreducible representations of the normal subgroup $\mathbb{Z}$ are labeled by $S^{1}=\{z \in \mathbb{C}|z|=1\}$ and given by $X^{n} \mapsto z^{n}$. The action of $\mathbb{Z} / 2$ is the involution given by conjugation $z \mapsto \bar{z}$. The quotient of $S^{1}$ by the $\mathbb{Z} / 2$ action is identified with the interval $[-1,1]$ by the map $z \mapsto \Re(z)$. For points inside the interval the corresponding irreducible representation of $\Gamma$ is two dimensional. At each of the two endpoints \pm 1 one gets two inequivalent irreducible representations of $\Gamma$. Thus we recover the picture of Example 4.2 and an isomorphism $C^{*}(\Gamma) \sim A$ where $A$ is the algebra (4.4).

The first two basic steps of the general theory are known for arbitrary discrete groups $\Gamma$, namely

1) The resolution of the diagonal and computation of the cyclic cohomology are provided by the geometric model (due to Burghelea [36]) given by the free loop space of the classifying space $B \Gamma$.

2) The assembly map (BC-map) of [15] from the K-homology of the classifying space $B \Gamma$ to the K-theory of the reduced $C^{*}$-algebra $C_{r}^{*}(\Gamma)$ is refined in [16] to take care of torsion in the group $\Gamma$ and gives a pretty good approximation to the K-theory of $C_{r}^{*}(\Gamma)(c f$. [185] and references therein).

In the presence of a natural smooth subalgebra of $C_{r}^{*}(\Gamma)$ containing the group ring and stable under holomorphic functional calculus, the combination of the two steps described above makes it possible to prove an index theorem which is an higher dimensional form of Atiyah's $L^{2}$-index theorem for coverings. This gave the first proof of the Novikov conjecture for hyperbolic groups $(80])$. Since then the analysis of dense smooth subalgebras has played a key role, in particular in the ground breaking work of Vincent Lafforgue. See [15], 121, 135, 185, 186.

The next step, i.e. the construction of a spectral geometry, is directly related to geometric group theory. In general one cannot expect to get a finite dimensional spectral triple since the growth properties of the group give (except for groups of polynomial growth) a basic obstruction ( $c f$. [59]). A general construction of a theta summable spectral triple was given in [54] Section IV.9. Basically the transition from finitely summable spectral triples to the theta summable ones is the transition from finite dimensional geometry to the infinite dimensional case. In the theta summable case the Chern character is no longer a finite dimensional cyclic cocycle and one needs to extend cyclic cohomology using cocycles with infinite support in the $(b, B)$ bicomplex fulfilling a subtle growth condition. The general theory of entire cyclic cohomology was developed in [60. It is in general quite difficult to compute the Chern character in the theta summable case and one had to wait quite a long time until it was done for the basic example of discrete subgroups of semi-simple Lie groups. This has been achieved in a remarkable recent paper of M. Puschnigg [169] in the case of real rank one. The fourth step i.e. the thermodynamics might seem irrelevant in the type II context of discrete groups. However a small variant of the construction of the group ring, namely the Hecke algebra associated to an almost normal inclusion of discrete groups (in the sense considered in [27]) suffices to meet the type III world. One of the open fields is to extend the above steps 1), 2) and 3) in the general context of almost normal inclusions of discrete groups, and to perform the thermodynamical analysis in the spirit of [6] in that context. 


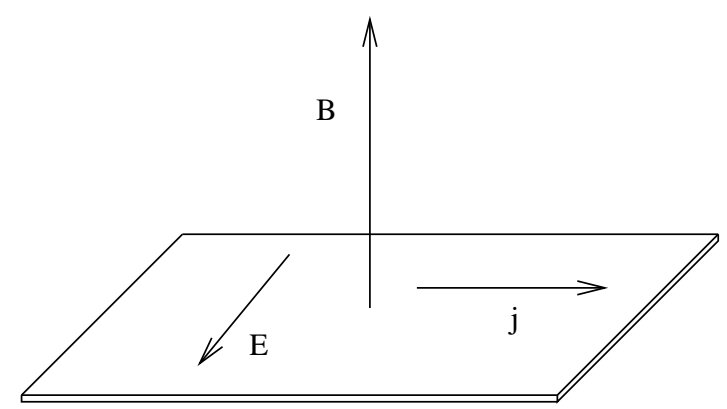

FiguRE 5. The quantum Hall effect experiment.

\section{Brillouin zone And the Quantum Hall efFect}

An important application to physics of the theory of non-commutative tori was the development of a rigorous mathematical model for the Integer Quantum Hall Effect (IQHE) obtained by Bellissard and collaborators [18, [19, [54].

The classical Hall effect is a physical phenomenon first observed in the XIX century [114. A very thin metal sample is immersed in a constant uniform strong magnetic field orthogonal to the surface of the sample. By forcing a constant current to flow through the sample, the flow of charge carriers in the metal is subject to a Lorentz force perpendicular to the current and the magnetic field. The equation for the equilibrium of forces in the sample is of the form

$$
N e \mathbf{E}+\mathbf{j} \wedge \mathbf{B}=0,
$$

where $\mathbf{E}$ is the electric field, $e$ and $N$ the charge and number of the charge carriers in the metal, $\mathbf{B}$ the magnetic field, and $\mathbf{j}$ the current.

The equation (8.1) defines a linear relation: the ratio of the intensity of the Hall current to the intensity of the electric field defines the Hall conductance,

$$
\sigma_{H}=\frac{N e \delta}{B},
$$

with $B=|\mathbf{B}|$ the intensity of the magnetic field and $\delta$ the sample width. The dimensionless quantity

$$
\nu_{H}=\frac{N \delta h}{B e}=\sigma_{H} R_{H}
$$

is called the filling factor, while the quantity $R_{H}=h / e^{2}$ is the Hall resistance. The filling factor measures the fraction of Landau level filled by conducting electrons in the sample. Thus, classically, the Hall conductance, measured in units of $e^{2} / h$, equals the filling factor. In 1980, about a century after the classical Hall effect was observed, von Klitzing's experiment showed that, lowering the temperature below $1 \mathrm{~K}$, the relation of Hall conductance to filling factor shows plateaux at integer values, 131. The integer values of the Hall conductance are observed with a surprising experimental accuracy of the order of $10^{-8}$. This phenomenon of quantization of the Hall conductance is known as Integer Quantum Hall Effect (IQHE). 
Laughlin first suggested that IQHE should be of a geometric origin [138. A detailed mathematical model of the IQHE, which accounts for all the important features of the experiment (quantization, localization, insensitivity to the presence of disorder, vanishing of direct conductance at plateaux levels) improving over the earlier Laughlin model, was developed by Bellissard and collaborators [18, [19].

Bellissard's approach to the IQHE is based on non-commutative geometry. The quantization of the Hall conductance at integer values is indeed geometric in nature: it resembles another well known "quantization" phenomenon that happens in the more familiar setting of the geometry of compact 2-dimensional manifolds, namely the Gauss-Bonnet theorem, where the integral of the curvature is an integer multiple of $2 \pi$, a property that is stable under deformations. In the same spirit, the values of the Hall conductance are related to the evaluation of a certain characteristic class, or, in other words, to an index theorem for a Fredholm operator.

More precisely, in the physical model one makes the simplifying assumption that the IQHE can be described by non-interacting particles. The Hamiltonian then describes the motion of a single electron subject to the magnetic field and an additional potential representing the lattice of ions in the conductor. In a perfect crystal and in the absence of a magnetic field, there is a group of translational symmetries. This corresponds to a group of unitary operators $U(a), a \in G$, where $G$ is the locally compact group of symmetries. Turning on the magnetic field breaks this symmetry, in the sense that translates of the Hamiltonian $H_{a}=U(a) H U(a)^{-1}$ no longer commute with the Hamiltonian $H$. Since there is no preferred choice of one translate over the others, the algebra of observables must include all translates of the Hamiltonian, or better their resolvents, namely the bounded operators

$$
R_{a}(z)=U(a)(z I-H)^{-1} U(a)^{-1} .
$$

For a particle of (effective) mass $m$ and charge $e$ confined to the plane, subject to a magnetic field of vector potential $\mathbf{A}$ and to a bounded potential $V$, the Hamiltonian is of the form

$$
H=\frac{1}{2 m} \sum_{j=1,2}\left(p_{j}-e A_{j}\right)^{2}+V=H_{0}+V,
$$

where the unperturbed part $H_{0}$ is invariant under the magnetic translations, namely the unitary representation of the translation group $\mathbb{R}^{2}$ given by

$$
U(a) \psi(x)=\exp \left(\frac{-i e B}{2 \hbar} \omega(x, a)\right) \psi(x-a),
$$

with $\omega$ the standard symplectic form in the plane. The hull (strong closure) of the translates (8.4) yields a topological space, whose homeomorphism type is independent of the point $z$ in the resolvent of $H$. This provides a non-commutative version of the Brillouin zone.

Recall that the Brillouin zones of a crystals are fundamental domains for the reciprocal lattice $\Gamma^{\sharp}$ obtained via the following inductive procedure. The Bragg hyperplanes of a crystal are the hyperplanes along which a pattern of diffraction of maximal intensity is observed when a beam of radiation (X-rays for instance) is shone at the crystal. The $N$-th Brillouin zone consists of all the points in (the dual) $\mathbb{R}^{d}$ such that the line from that point to the origin crosses exactly $(n-1)$ Bragg hyperplanes of the crystal. 


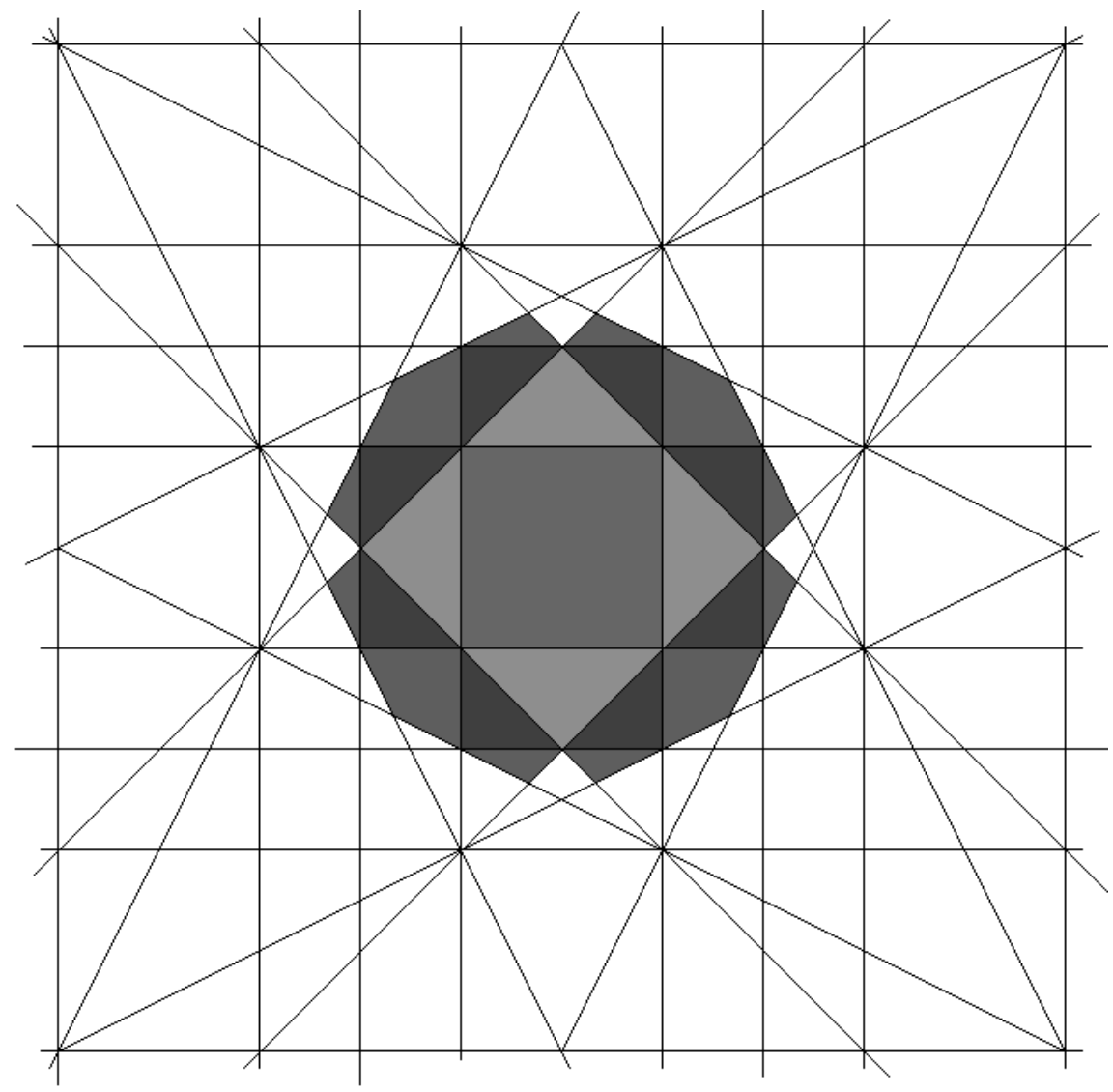

FiguRE 6. Brillouin zones for a 2-dimensional crystal

More precisely, in our case, if $e_{1}$ and $e_{2}$ are generators of the periodic lattice, we obtain a commutation relation

$$
U\left(e_{1}\right) U\left(e_{2}\right)=e^{2 \pi i \theta} U\left(e_{2}\right) U\left(e_{1}\right),
$$

where $\theta$ is the flux of the magnetic field through a fundamental domain for the lattice, in dimensionless units, hence the non-commutative Brillouin zone is described by a noncommutative torus.

This can also be seen easily in a discrete model, where the Hamiltonian is given by an operator

$$
\begin{aligned}
\left(H_{a} f\right)(m, n) & =e^{-i a_{1} n} f(m+1, n)+\quad e^{i a_{2} n} f(m-1, n) \\
& +e^{-i a_{2} m} f(m, n+1)+\quad e^{i a_{2} m} f(m, n-1),
\end{aligned}
$$

for $f \in L^{2}\left(\mathbb{Z}^{2}\right)$. This is a discrete version of the magnetic Laplacian. Notice then that (8.6) can be written in the form

$$
H_{a}=U+V+U^{*}+V^{*}
$$

for

$$
(U f)(m, n)=e^{-i a_{2} m} f(m, n+1) \quad(V f)(m, n)=e^{-i a_{1} n} f(m+1, n) .
$$




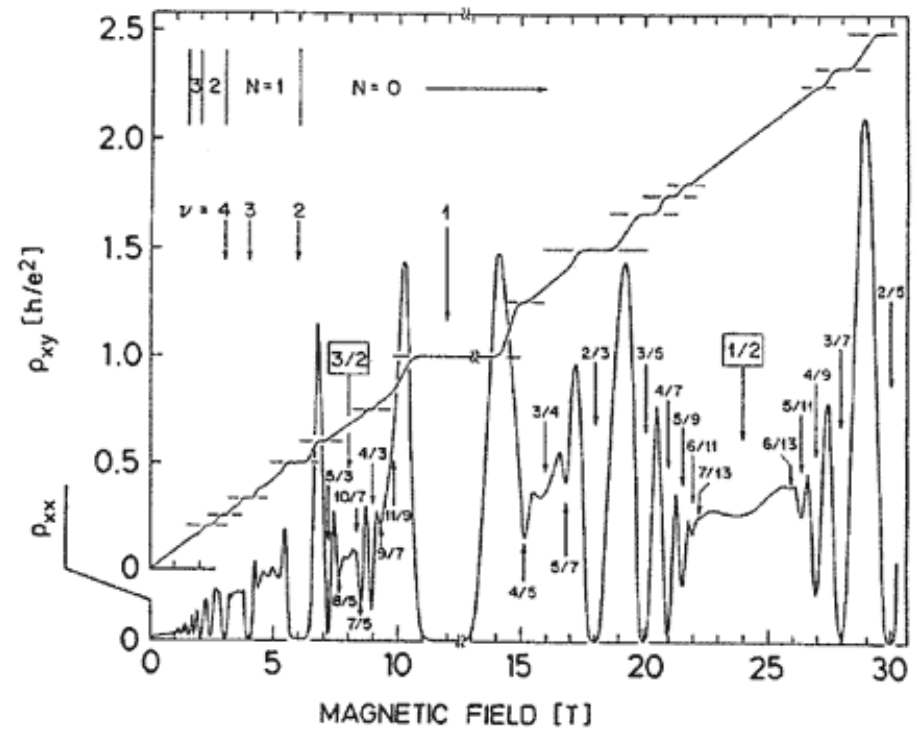

FiguRE 7. Observed fractions in the quantum Hall effect.

These clearly satisfy the commutation relation (6.12) of $\mathbb{T}_{\theta}^{2}$ with $\theta=a_{2}-a_{1}$. In the zero-temperature limit, the Hall conductance satisfies the Kubo formula

$$
\sigma_{H}=\frac{1}{2 \pi i R_{H}} \tau\left(P_{\mu}\left[\delta_{1} P_{\mu}, \delta_{2} P_{\mu}\right]\right)
$$

where $P_{\mu}$ is a spectral projection of the Hamiltonian on energies smaller or equal to the Fermi level $E_{\mu}, \tau$ is the trace on $\mathcal{A}_{\theta}$ given by

$$
\tau\left(\sum a_{n, m} U^{n} V^{m}\right)=a_{0,0} .
$$

and $\delta_{1}, \delta_{2}$ are as in (6.15). Here we assume that the Fermi level $\mu$ is in a gap in the spectrum of the Hamiltonian. Then the spectral projections $P_{\mu}$ belong to the $C^{*}$-algebra of observables. The Kubo formula (8.7) can be derived from purely physical considerations, such as transport theory and the quantum adiabatic limit.

The main result then is the fact that the integrality of the conductance observed in the Integer Quantum Hall Effect is explained topologically, that is, in terms of the integrality of the cyclic cocycle $\tau\left(a^{0}\left(\delta_{1} a^{1} \delta_{2} a^{2}-\delta_{2} a^{1} \delta_{1} a^{2}\right)\right)$ (cf. [57]).

The fractional QHE was discovered by Stormer and Tsui in 1982. The setup is as in the quantum Hall effect: in a high quality semi-conductor interface, which will be modelled by an infinite 2-dimensional surface, with low carrier concentration and extremely low temperatures $\sim 10 \mathrm{mK}$, in the presence of a very strong magnetic field, the experiment shows that the graph of $\frac{h}{e^{2}} \sigma_{H}$ against the filling factor $\nu$ exhibits plateaux at certain fractional values.

The independent electron approximation that, in the case of the integer quantum Hall effect, reduces the problem to a single electron wavefunction is no longer viable in this case and one 
has to incorporate the Coulomb interaction between the electrons in a many-electron theory. Nonetheless, it is possible to use a crude approximation, whereby one alters the underlying geometry to account for an average effect of the multi-electron interactions. One can obtain this way a model of the fractional quantum Hall effect via noncommutative geometry ( $c f$. [152, 153, [154]), where one uses hyperbolic geometry to simulate the interactions.

The noncommutative geometry approach to the quantum Hall effect described above was extended to hyperbolic geometry in [37. The analog of the operator (8.6) is given by the Harper operator on the Cayley graph of a finitely generated discrete subgroup $\Gamma$ of $\mathrm{PSL}_{2}(\mathbb{R})$. Given $\sigma: \Gamma \times \Gamma \rightarrow U(1)$ satisfying $\sigma(\gamma, 1)=\sigma(1, \gamma)=1$ and

$$
\sigma\left(\gamma_{1}, \gamma_{2}\right) \sigma\left(\gamma_{1} \gamma_{2}, \gamma_{3}\right)=\sigma\left(\gamma_{1}, \gamma_{2} \gamma_{3}\right) \sigma\left(\gamma_{2}, \gamma_{3}\right)
$$

one considers the right $\sigma$-regular representation on $\ell^{2}(\Gamma)$ of the form

$$
R_{\gamma}^{\sigma} \psi\left(\gamma^{\prime}\right)=\psi\left(\gamma^{\prime} \gamma\right) \sigma\left(\gamma^{\prime}, \gamma\right)
$$

satisfying

$$
R_{\gamma}^{\sigma} R_{\gamma^{\prime}}^{\sigma}=\sigma\left(\gamma, \gamma^{\prime}\right) R_{\gamma \gamma^{\prime}}^{\sigma}
$$

For $\left\{\gamma_{i}\right\}_{i=1}^{r}$ a symmetric set of generators of $\Gamma$, the Harper operator is of the form

$$
\mathcal{R}_{\sigma}=\sum_{i=1}^{r} R_{\gamma_{i}}^{\sigma}
$$

and the operator $r-\mathcal{R}_{\sigma}$ is the discrete analog of the magnetic Laplacian ( $c f$. 194]).

The idea is that, by effect of the strong interaction with the other electrons, a single electron "sees" the surrounding geometry as hyperbolic, with lattice sites that appear (as a multiple image effect) as the points in a lattice $\Gamma \subset \mathrm{PSL}_{2}(\mathbb{R})$. Thus, one considers the general form of such a lattice

$$
\Gamma=\Gamma\left(g ; \nu_{1}, \ldots, \nu_{n}\right),
$$

with generators $a_{i}, b_{i}, c_{j}$, with $i=1, \ldots, g$ and $j=1, \ldots, n$ and a presentation of the form

$$
\Gamma\left(g ; \nu_{1}, \ldots, \nu_{n}\right)=\left\langle a_{i}, b_{i}, c_{j} \mid \prod_{i=1}^{g}\left[a_{i}, b_{i}\right] c_{1} \cdots c_{n}=1, \quad c_{j}^{\nu_{j}}=1\right\rangle .
$$

The quotient of the action of $\Gamma$ by isometrieson $\mathbb{H}$,

$$
\Sigma\left(g ; \nu_{1}, \ldots, \nu_{n}\right):=\Gamma \backslash \mathbb{H},
$$

is a hyperbolic orbifold.

Let $P_{E}$ denote denote the spectral projection associated to the Fermi level, i.e. $P_{E}=$ $\chi_{(-\infty, E]}(H)$. Then, in the zero temperature limit, the Hall conductance is given by

$$
\sigma_{E}=\operatorname{tr}_{K}\left(P_{E}, P_{E}, P_{E}\right)
$$

where $\operatorname{tr}_{K}$ denotes the conductance 2-cocycle. It is a cyclic 2-cocycle on the twisted group algebra $\mathbb{C}(\Gamma, \sigma)$ of the form

$$
\operatorname{tr}_{K}\left(f_{0}, f_{1}, f_{2}\right)=\sum_{j=1}^{g} \operatorname{tr}\left(f_{0}\left(\delta_{j}\left(f_{1}\right) \delta_{j+g}\left(f_{2}\right)-\delta_{j+g}\left(f_{1}\right) \delta_{j}\left(f_{2}\right)\right)\right)
$$




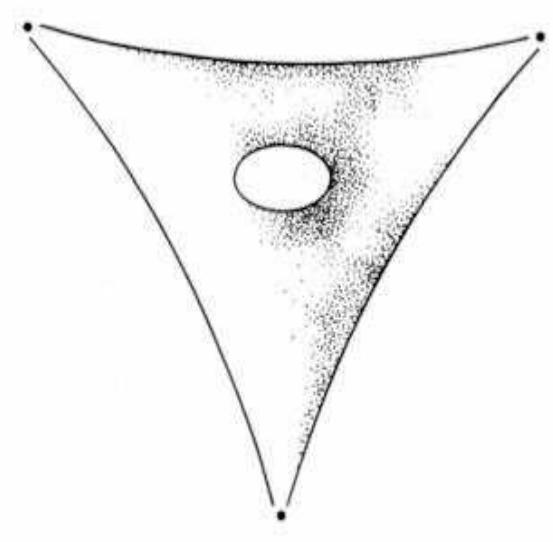

Figure 8. Hyperbolic orbifolds.

where the $\delta_{j}$ are derivations associated to the 1-cocycles $a_{j}$ associated to a symplectic basis $\left\{a_{j}, b_{j}\right\}_{j=1, \ldots, g}$ of $H^{1}(\Gamma, \mathbb{R})(c f .[154])$.

Within this model, one obtains the fractional values of the Hall conductance as integer multiples of orbifold Euler characteristics

$$
\chi_{\text {orb }}\left(\Sigma\left(g ; \nu_{1}, \ldots, \nu_{n}\right)\right)=2-2 g+\nu-n \in \mathbb{Q} .
$$

In fact, one shows ( $c f$. 153, 154) that the conductance 2-cocycle is cohomologous to another cocycle, the area 2-cocycle, for which one can compute the values on $K$-theory (hence the value of (8.14) ) by applying a twisted version of the Connes-Moscovici higher index theorem 80.

While in the case of the integer quantum Hall effect the noncommutative geometry model is completely satisfactory and explains all the physical properties of the system, in the fractional case the orbifold model can be considered as a first rough approximation to the quantum field theory that coverns the fractional quantum Hall effect. For instance, the geometry of 2-dimensional hyperbolic orbifolds is related to Chern-Simons theory through the moduli spaces of vortex equations. This remains an interesting open question.

\section{Tilings}

In general, by a tiling $\mathcal{T}$ in $\mathbb{R}^{d}$ one means the following. One considers a finite collection $\left\{\tau_{1}, \ldots, \tau_{N}\right\}$ of closed bounded subsets of $\mathbb{R}^{d}$ homeomorphic to the unit ball. These are called the prototiles. One usually assumes that the prototiles are polytopes in $\mathbb{R}^{d}$ with a single $d$-dimensional cell which is the interior of the prototile, but this assumption can be relaxed. A tiling $\mathcal{T}$ of $\mathbb{R}^{d}$ is then a covering of $\mathbb{R}^{d}$ by sets with disjoint interior, each of which is a tile, that is, a translate of one of the prototiles. 


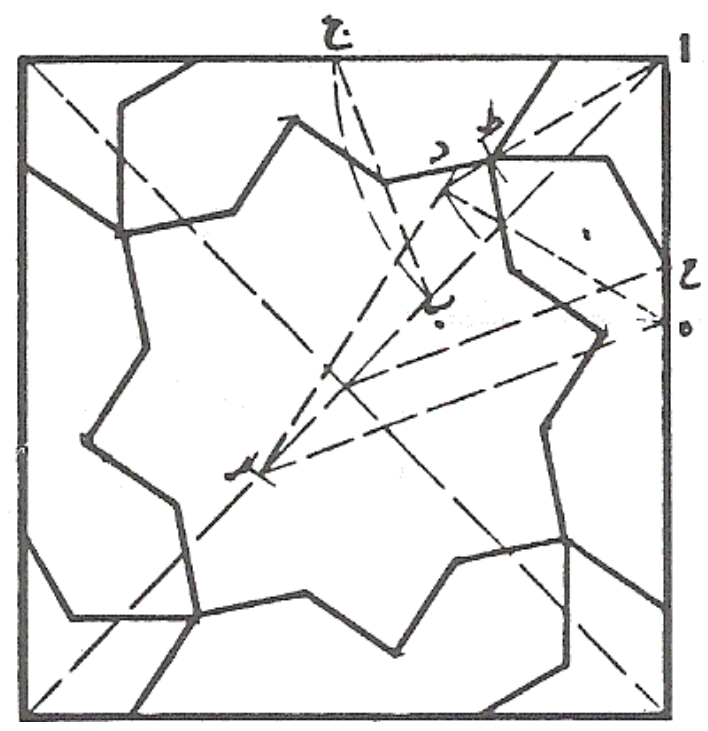

Figure 9. Prototiles for tilings.

Given a tiling $\mathcal{T}$ of $\mathbb{R}^{d}$ one can form its orbit closure under translations. The metric on tilings is defined by saying that two tilings are close if they almost agree on a large ball centered at the origin in $\mathbb{R}^{d}$ (for more details and equivalent definitions see e.g. [4], [20]).

Tilings can be periodic or aperiodic. There are many familiar examples of periodic tilings, while the best known examples of aperiodic tilings are the Penrose tilings [165. Similar types of aperiodic tilings have been widely studied in the physics of quasicrystals (cf. e.g. [13, [20]). It was understood very early on in the development of noncommutative geometry ( $c f$. 59 . and pp.5-7, pp.88-93, and pp.175-178 of [54]) that Penrose tilings provide an interesting class of noncommutative spaces.

In fact, one can consider on the set $\Omega$ of tilings $\mathcal{T}$ with given prototiles $\left\{\tau_{1}, \ldots, \tau_{N}\right\}$ the equivalence relation given by the action of $\mathbb{R}^{d}$ by translations, i.e. one identifies tilings that can be obtained from one another by translations. In the case of aperiodic tilings, this yields the type of quotient construction described in Section 4, which leads naturally to noncommutative spaces. An explicit description of this noncommutative space for the case of Penrose tilings can be found in $\S$ II.3 of [54].

To simplify the picture slightly, we can consider the similar problem (dually) with arrangements of points in $\mathbb{R}^{d}$ instead of tilings. This is the formulation used in the theory of aperiodic solids and quasicrystals ( $c f$. [20]). Then, instead of tilings $\mathcal{T}$, we consider discrete subsets of points $\mathcal{L} \subset \mathbb{R}^{d}$. Such $\mathcal{L}$ is a Delauney set if there are radii $r, R>0$ such that every open ball of radius $r$ meets $\mathcal{L}$ in at most one point and every closed ball of radius $R$ meets $\mathcal{L}$ in at least one point. One can describe $\mathcal{L}$ by the counting measure

$$
\mu_{\mathcal{L}}(f)=\sum_{x \in \mathcal{L}} f(x)
$$




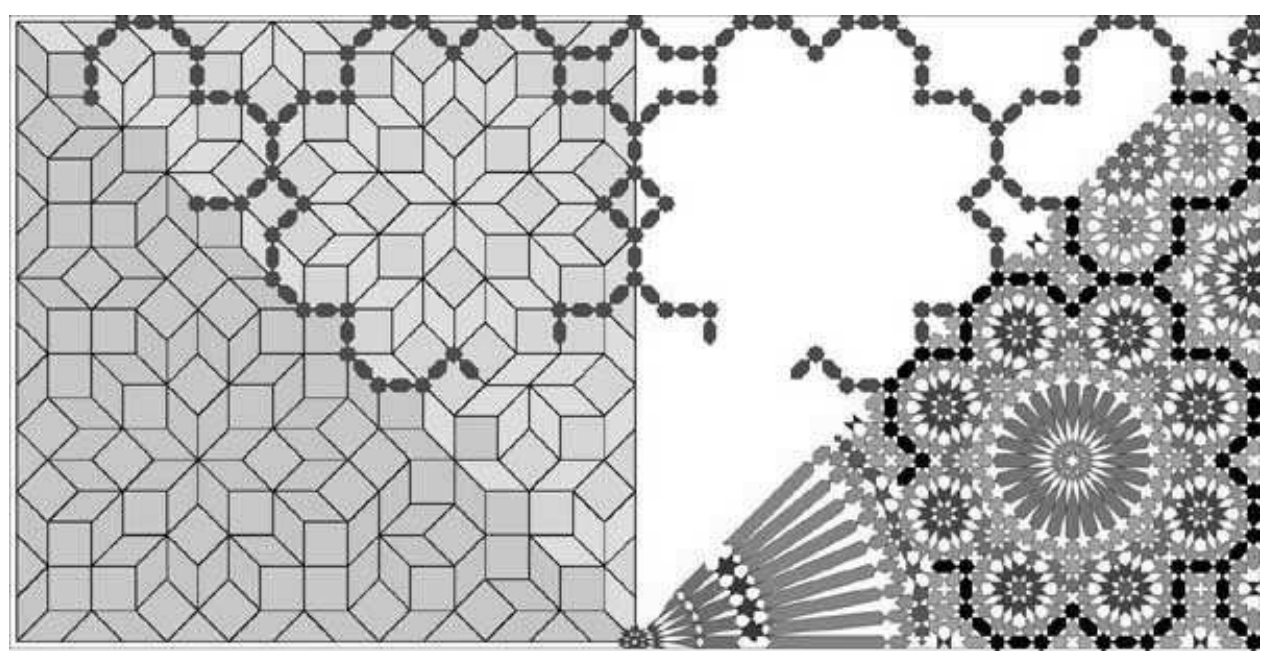

FiguRE 10. Quasiperiodic tilings and zellijs.

and one can take the orbit closure $\Omega$ of the action of $\mathbb{R}^{d}$ by translations

$$
\mu_{\mathcal{L}} \mapsto T_{-a} \mu_{\mathcal{L}}=\mu_{\mathcal{L}} \circ T_{a}, \quad \text { for } a \in \mathbb{R}^{d},
$$

in the space $\mathcal{M}\left(\mathbb{R}^{d}\right)$ of Radon measures with the weak* topology. The Hull of $\mathcal{L}$ is the dynamical system $(\Omega, T)$, where $T$ denotes the action of $\mathbb{R}^{d}$ by translations.

This dynamical system determines a corresponding noncommutative space, describing the quotient of $\Omega$ by translations, namely the crossed product $C^{*}$-algebra

$$
\mathcal{A}=C(\Omega) \rtimes_{T} \mathbb{R}^{d} .
$$

In fact, one can also consider the groupoid with set of units the transversal

$$
X=\{\omega \in \Omega: 0 \in \operatorname{Support}(\omega)\},
$$

arrows of the form $(\omega, a) \in \Omega \times \mathbb{R}^{d}$, with source and range maps $s(\omega, a)=T_{-a} \omega, r(\omega, a)=\omega$ and $(\omega, a) \circ\left(T_{-a} \omega, b\right)=(\omega, a+b)(c f$. 20] $)$. This defines a locally compact groupoid $\mathcal{G}(\mathcal{L}, X)$. The $C^{*}$-algebras $C^{*}(\mathcal{G}(\mathcal{L}, X))$ and $C(\Omega) \rtimes_{T} \mathbb{R}^{d}$ are Morita equivalent.

In the case where $\mathcal{L}$ is a periodic arrangement of points with cocompact symmetry group $\Gamma \subset \mathbb{R}^{d}$, the space $\Omega$ is an ordinary commutative space, which is topologically a torus $\Omega=$ $\mathbb{R}^{d} / \Gamma$. The $C^{*}$-algebra $\mathcal{A}$ is in this case isomorphic to $C(\hat{\Gamma}) \otimes \mathcal{K}$, where $\mathcal{K}$ is the algebra of compact operators and $\hat{\Gamma}$ is the Pontrjagin dual of the abelian group $\Gamma \cong \mathbb{Z}^{d}$, isomorphic to $T^{d}$, obtained by taking the dual of $\mathbb{R}^{d}$ modulo the reciprocal lattice

$$
\Gamma^{\sharp}=\left\{k \in \mathbb{R}^{d}:\langle k, \gamma\rangle \in 2 \pi \mathbb{Z}, \forall \gamma \in \Gamma\right\} .
$$

Thus, in physical language, $\hat{\Gamma}$ is identified with the Brillouin zone $B=\mathbb{R}^{d} / \gamma^{\sharp}$ of the periodic crystal $\mathcal{L}$ (cf. Section $[$ ). In this periodic case, the transversal $X=\mathcal{L} / \Gamma$ is a finite set of points. The groupoid $C^{*}$-algebra $C^{*}(\mathcal{G}(\mathcal{L}, X))$ is in this case isomorphic to $C(\hat{\Gamma}) \otimes M_{k}(\mathbb{C})$, where $k$ is the cardinality of the transversal $X$. Thus, the periodic case falls back into the realm of commutative spaces, while the aperiodic patterns give rise to truly noncommutative spaces, which are highly nontrivial and interesting. 

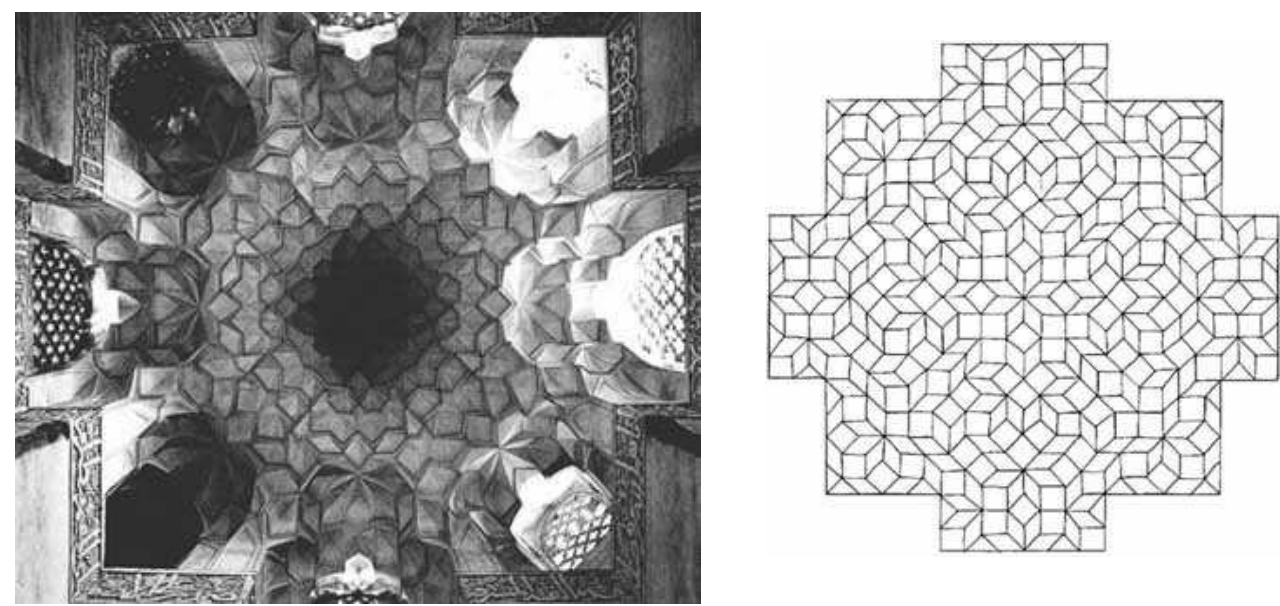

FiguRE 11. Quasiperiodic tilings and muqarnas.

One of the richest sources of interesting tilings are the zellijs and muqarnas widely used in ancient architecture. Also collectively defined as "arabesques", not only these patterns exhibit highly nontrivial geometries, but they reflect the intricate interplay between philosophy, mathematics, and aesthetics ( $c f$. 9], 35]). Some of the best studies on zellijs and muqarnas concentrate on 2-dimensional periodic patterns. For instance we find in [9], p.43:

"As Nature is based on rhythm, so the arabesque is rhythmic in concept. It reflects movement marked by the regular recurrence of features, elements, phenomena; hence it has periodicity."

It seems from this viewpoint that only the theory of periodic tilings (i.e. commutative geometry) should be relevant in this context. However, more recent studies ( $c f$. [35], [40, [41, 162]) suggest that the design of zellijs and muqarnas was not limited to 2-dimensional crystallographic groups, but, especially during the Timurid period, it involved also aperiodic patterns with fivefold symmetry, analogous to those observed in quasi-crystals. This is no accident and was certainly the result of a highly developed geometric theory: already in the historic textbook of Abu'l-Wafa' al-Buzjani (940-998) on geometric constructions 198 there is explicit mention of meetings and discussions where mathematicians were directly involved alongside artisans in the design of arabesque patterns.

The appearance of aperiodic tilings is documented in the anonymous Persian manuscript [5] "On interlocking similar and congruent figures", which dates back to the 11th-13th century. Some of these aperiodic aspects of zellijs and muqarnas were studied by Bulatov in the book [35, which also contains Vil'danova's Russian translation of the ancient Persian text.

\section{Noncommutative SPACES FROM DYNAMiCAL SySTEMS}

We will look at some examples of noncommutative spaces associated to a discrete dynamical system $T$, for instance given by a self mapping of a Cantor set. Such noncommutative spaces have been extensively studied in a series of papers ( $c f$. [107] and [187] for a survey) where 
C. Skau and his coworkers have obtained remarkable results on the classification of minimal actions of $\mathbb{Z}$ on Cantor sets using the $K$-theory of the associated $C^{*}$-algebra.

It was found recently ( $c f$. .91, [92, $\S 4$ of [149] and $\S 8$ of [148]) that the mapping torus of such systems can be used to model the "dual graph" of the fibers at the archimedean primes of arithmetic surfaces, in Arakelov geometry, in the particular case in which the dynamical system $T$ is a subshift of finite type encoding the action of a Schottky group $\Gamma \subset \mathrm{SL}_{2}(\mathbb{C})$ on its limit set $\Lambda_{\Gamma} \subset \mathbb{P}^{1}(\mathbb{C})$. In fact, the results of [91] were motivated by earlier results of Manin 145] that provided a geometric model for such dual graphs in terms of hyperbolic geometry and Schottky uniformizations.

More generally, given an alphabet with letters $\left\{\ell_{1}, \ldots, \ell_{N}\right\}$, the space $\mathcal{S}_{A}^{+}$of a subshift of finite type consists of all right-infinite admissible sequences

$$
w=a_{0} a_{1} a_{2} \ldots a_{n} \ldots
$$

in the letters of the alphabet. Namely, $a_{i} \in\left\{\ell_{1}, \ldots, \ell_{N}\right\}$ subject to an admissibility condition specified by an $N \times N$ matrix $A$ with entries in $\{0,1\}$. Two letters $\ell_{i}$ and $\ell_{j}$ in the alphabet can appear as consecutive digits $a_{k}, a_{k+1}$ in the word $w$ if and only if the entry $A_{i j}$ of the admissibility matrix $A$ is equal to 1 . One defines similarly the space $\mathcal{S}_{A}$ as the set of doubly-infinite admissible sequences

$$
w=\ldots a_{-m} \ldots a_{-2} a_{-1} a_{0} a_{1} a_{2} \ldots a_{n} \ldots
$$

The sets $\mathcal{S}_{A}^{+}$and $\mathcal{S}_{A}$ have a natural choice of a topology. In fact, on $\mathcal{S}_{A}$ we can put the topology generated by the sets $W^{s}(x, \ell)=\left\{y \in \mathcal{S}_{A} \mid x_{k}=y_{k}, k \geq \ell\right\}$, and the $W^{u}(x, \ell)=\{y \in$ $\left.\mathcal{S}_{A} \mid x_{k}=y_{k}, k \leq \ell\right\}$ for $x \in \mathcal{S}_{A}$ and $\ell \in \mathbb{Z}$. This induces a topology with analogous properties on $\mathcal{S}_{A}^{+}$by realizing it as a subset of $\mathcal{S}_{A}$, for instance, by extending each sequence to the left as a constant sequence. One then considers on $\mathcal{S}_{A}$ (or on $\mathcal{S}_{A}^{+}$) the action of the two-sided (resp. one-sided) shift $T$ defined by $(T w)_{k}=a_{k+1}$, where the $a_{k}$ are the digits of the word $w$. Namely, the one-sided shift on $\mathcal{S}_{A}^{+}$is of the form

$$
T\left(a_{0} a_{1} a_{2} \ldots a_{\ell} \ldots\right)=a_{1} a_{2} \ldots a_{\ell} \ldots
$$

while the two-sided shift on $\mathcal{S}_{A}$ acts as

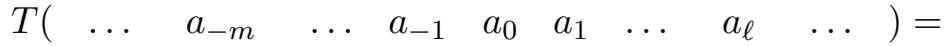

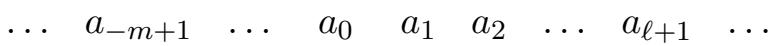

Tipically spaces $\mathcal{S}_{A}^{+}$and $\mathcal{S}_{A}$ are topologically Cantor sets. The one-sided shift $T$ of (10.3) is a continuous surjective map on $\mathcal{S}_{A}^{+}$, while the two-sided shift $T$ of (10.4) is a homeomorphism of $\mathcal{S}_{A}$.

For example, let $\Gamma$ be a free group in $g$ generators $\left\{\gamma_{1}, \ldots, \gamma_{g}\right\}$. Consider the alphabet $\left\{\gamma_{1}, \ldots, \gamma_{g}, \gamma_{1}^{-1}, \ldots, \gamma_{g}^{-1}\right\}$. Then one can consider the right-infinite, or doubly-infinite words in these letters, without cancellations, that is, subject to the admissibility rule that $a_{k+1} \neq$ $a_{k}^{-1}$. This defines a subshift of finite type where the matrix $A$ is the symmetric $2 g \times 2 g$ matrix with $A_{i j}=0$ for $|i-j|=g$ and $A_{i j}=1$ otherwise. Suppose that $\Gamma$ is a Schottky group of genus $g$, i.e. a finitely generated discrete subgroup $\Gamma \subset \mathrm{SL}_{2}(\mathbb{C})$, isomorphic to a free group in $g$ generators, where all nontrivial elements are hyperbolic. Then the points in $\mathcal{S}_{A}^{+}$ parameterize points in the limit set $\Lambda_{\Gamma} \subset \mathbb{P}^{1}(\mathbb{C})$ (the set of accumulation points of orbits of 


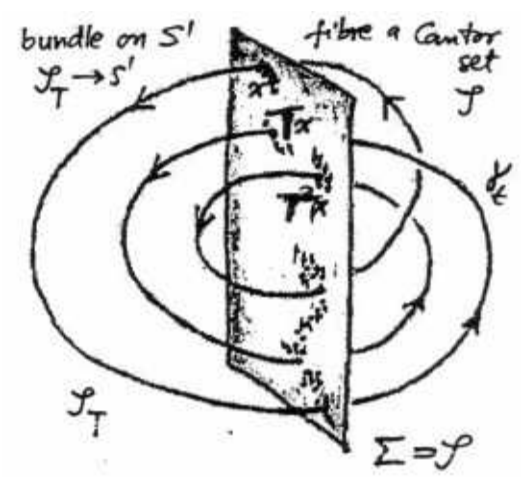

FiguRE 12. Mapping Torus

$\Gamma)$. The points in $\mathcal{S}_{A}$ parameterize geodesics in the three dimensional real hyperbolic space $\mathbb{H}^{3}$ with ends at points on the limit set $\Lambda_{\Gamma}$.

The pair $\left(\mathcal{S}_{A}, T\right)$ is a typical example of an interesting class of dynamical systems, namely it is a Smale space. This means that locally $\mathcal{S}_{A}$ can be decomposed as the product of expanding and contracting directions for $T$. Namely, the following properties are satisfied.

- For every point $x \in \mathcal{S}_{A}$ there exist subsets $W^{s}(x)$ and $W^{u}(x)$ of $\mathcal{S}_{A}$, such that $W^{s}(x) \times W^{u}(x)$ is homeomorphic to a neighborhood of $x$.

- The map $T$ is contracting on $W^{s}(x)$ and expanding on $W^{u}(x)$, and $W^{s}(T x)$ and $T\left(W^{s}(x)\right)$ agree in some neighborhood of $x$, and so do $W^{u}(T x)$ and $T\left(W^{u}(x)\right)$.

A construction of Ruelle shows that one can associate different $C^{*}$-algebras to Smale spaces (cf. [180, 170, 171]). For Smale spaces like $\left(\mathcal{S}_{A}, T\right)$ there are four basic possibilities: the crossed product algebra $C\left(\mathcal{S}_{A}\right) \rtimes_{T} \mathbb{Z}$ and the $\mathrm{C}^{*}$-algebras $\mathrm{C}^{*}\left(\mathcal{G}^{s}\right) \rtimes_{T} \mathbb{Z}, \mathrm{C}^{*}\left(\mathcal{G}^{u}\right) \rtimes_{T} \mathbb{Z}, \mathrm{C}^{*}\left(\mathcal{G}^{a}\right) \rtimes_{T}$ $\mathbb{Z}$ obtained by considering the action of the shift $T$ on the groupoid $\mathrm{C}^{*}$-algebra associated to the groupoids $\mathcal{G}^{s}, \mathcal{G}^{u}, \mathcal{G}^{a}$ of the stable, unstable, and asymptotic equivalence relations on $\left(\mathcal{S}_{A}, T\right)$.

The first choice, $C\left(\mathcal{S}_{A}\right) \rtimes_{T} \mathbb{Z}$, is closely related to the continuous dynamical system given by the mapping torus of $T$, while a choice like $\mathrm{C}^{*}\left(\mathcal{G}^{u}\right) \rtimes_{T} \mathbb{Z}$ is related to the "bad quotient" of $\mathcal{S}_{A}^{+}$by the action of $T$. In the example of the Schottky group this corresponds to the action of $\Gamma$ on its limit set.

One can consider the suspension flow $\mathcal{S}_{T}$ of a dynamical system $T$, that is, the mapping torus of the dynamical system $\left(\mathcal{S}_{A}, T\right)$, which is defined as

$$
\mathcal{S}_{T}:=\mathcal{S}_{A} \times[0,1] /(x, 0) \sim(T x, 1) .
$$

The first cohomology group of $\mathcal{S}_{T}$ is the "ordered cohomology" of the dynamical system $T$, in the sense of [30] 164]. There is an identification of $H^{1}\left(\mathcal{S}_{T}, \mathbb{Z}\right)$ with the $K_{0}$-group of the crossed product $\mathrm{C}^{*}$-algebra for the action of $T$ on $\mathcal{S}_{A}$,

$$
H^{1}\left(\mathcal{S}_{T}, \mathbb{Z}\right) \cong K_{0}\left(\mathrm{C}\left(\mathcal{S}_{A}\right) \rtimes_{T} \mathbb{Z}\right) .
$$


This can be seen from the Pimsner-Voiculescu exact sequence ( $c f$. [167]) for the $K$-theory of a crossed product by $\mathbb{Z}$, which in this case reduces to

$$
0 \rightarrow K_{1}\left(\mathrm{C}(\mathcal{S}) \rtimes_{T} \mathbb{Z}\right) \rightarrow \mathrm{C}(\mathcal{S}, \mathbb{Z}) \stackrel{I-T_{*}}{\rightarrow} \mathrm{C}(\mathcal{S}, \mathbb{Z}) \rightarrow K_{0}\left(\mathrm{C}(\mathcal{S}) \rtimes_{T} \mathbb{Z}\right) \rightarrow 0,
$$

It can also be seen in terms of the Thom isomorphism of [55], [56].

In fact, as we discussed in Section 2, one of the fundamental construction of noncommutative geometry (cf. [56]) is that of homotopy quotients. These are commutative spaces, which provide, up to homotopy, geometric models for the corresponding noncommutative spaces. The noncommutative spaces themselves, as we are going to show in our case, appear as quotient spaces of foliations on the homotopy quotients with contractible leaves.

For the noncommutative space $\mathcal{S}_{A} / \mathbb{Z}$, with $\mathbb{Z}$ acting as powers of the invertible two-sided shift, the homotopy quotient is precisely the mapping torus (10.5),

$$
\mathcal{S}_{T}=\mathcal{S} \times_{\mathbb{Z}} \mathbb{R} .
$$

The noncommutative space $\mathcal{S} / \mathbb{Z}$ can be identified with the quotient space of the natural foliation on (10.8) whose generic leaf is contractible (a copy of $\mathbb{R}$ ).

Another noncommutative space associated to a subshift of finite type $T$ (which, up to Morita equivalence, correspond to another choice of the $C^{*}$-algebra of a Smale space, as mentioned above) is the Cuntz-Krieger algebra $\mathcal{O}_{A}$, where $A$ is the admissibility matrix of the subshift finite type ( $c f$. 94, 95]).

A partial isometry is a linear operator $S$ satisfying the relation $S=S S^{*} S$. The CuntzKrieger algebra $\mathcal{O}_{A}$ is defined as the universal $C^{*}$-algebra generated by partial isometries $S_{1}, \ldots, S_{N}$, satisfying the relations

$$
\begin{gathered}
\sum_{j} S_{j} S_{j}^{*}=I \\
S_{i}^{*} S_{i}=\sum_{j} A_{i j} S_{j} S_{j}^{*} .
\end{gathered}
$$

In the case of a Schottky group $\Gamma \subset \mathrm{PSL}_{2}(\mathbb{C})$ of genus $g$, the Cuntz-Krieger algebra $\mathcal{O}_{A}$ can be described in terms of the action of the free group $\Gamma$ on its limit set $\Lambda_{\Gamma} \subset \mathbb{P}^{1}(\mathbb{C})$ ( $c f$. [177, [190]), so that we can regard $\mathcal{O}_{A}$ as a noncommutative space replacing the classical quotient $\Lambda_{\Gamma} / \Gamma$

$$
\mathcal{O}_{A} \cong C\left(\Lambda_{\Gamma}\right) \rtimes \Gamma
$$

The quotient space

$$
\Lambda_{\Gamma} \times_{\Gamma} \mathbb{H}^{3}=\Lambda_{\Gamma} \times_{\Gamma} \underline{E} \Gamma,
$$

is precisely the homotopy quotient of $\Lambda_{\Gamma}$ with respect to the action of $\Gamma$, with $\underline{E} \Gamma=\mathbb{H}^{3}$ and the classifying space $\underline{B} \Gamma=\mathbb{H}^{3} / \Gamma$. Here $\mathbb{H}^{3} / \Gamma$ is a hyperbolic 3-manifold of infinite volume, which is topologically a handlebody of genus $g$. In this case also we find that the noncommutative space $\Lambda_{\Gamma} / \Gamma$ is the quotient space of a foliation on the homotopy quotient (10.12) with contractible leaves $\mathbb{H}^{3}$. 


\section{NonCOMMUtATIVE SPACES FROM STRING THEORY}

The main aspects of string and D-brane theory that involve noncommutative geometry are the bound states of configurations of parallel D-branes [201, the matrix models for M-theory [14] and the strong coupling limit of string theory ( $c f$. e.g. [7, 8]). It also plays an important role in the M-theory compactifications 68. We shall not discuss all these aspects in detail here. Since the focus of this review is on examples we only mention a couple of examples of noncommutative spaces arising from string and D-brane theory.

The noncommutative tori and the components of the Yang-Mills connections appear in the classification of the BPS states in M-theory 68.

Recall first that Yang-Mills theory on noncommutative tori can be formulated ( $c f$. 88]) using suitable notions of connections and curvature for noncommutative spaces. In fact, the analogs of connection and curvature of vector bundles are straightforward to obtain ([57]): a connection is just given by the associated covariant differentiation $\nabla$ on the space of smooth sections. Thus here it is given by a pair of linear operators,

$$
\nabla_{j}: \mathcal{S}(\mathbb{R}) \rightarrow \mathcal{S}(\mathbb{R})
$$

such that

$$
\nabla_{j}(\xi b)=\left(\nabla_{j} \xi\right) b+\xi \delta_{j}(b) \quad \forall \xi \in \mathcal{S}, b \in \mathcal{A}_{\theta} .
$$

One checks that, as in the usual case, the trace of the curvature

$$
\Omega=\nabla_{1} \nabla_{2}-\nabla_{2} \nabla_{1}
$$

is independent of the choice of the connection.

We can make the following choice for the connection:

$$
\left(\nabla_{1} \xi\right)(s)=-\frac{2 \pi i s}{\theta} \xi(s) \quad\left(\nabla_{2} \xi\right)(s)=\xi^{\prime}(s) .
$$

Notice that, up to the correct powers of $2 \pi i$, the total curvature of $\mathcal{S}$ is an integer. In fact, the curvature $\Omega$ is constant, equal to $\frac{1}{\theta}$, so that the irrational number $\theta$ disappears in the total curvature, $\theta \times \frac{1}{\theta}$. This integrality phenomenon, where the pairing of dimension and curvature (both of which are non-integral) yields an integer:

$$
\operatorname{dim} \times \Omega \sim \theta \times \frac{1}{\theta}=\text { integer, }
$$

is the basis for the development of a theory of characteristic classes for non-commutative spaces. In the general case, this requires the development of more sophisticated tools, since analogs of the derivations $\delta_{i}$ used in the case of the noncommutative tori are not there in general. The general theory is obtained through cyclic homology, as developed in [58.

Consider then the modules $\mathcal{H}_{p, q}^{\theta}$ described in Section [6] It is possible to define an $\mathcal{A}_{\theta}$ valued inner product $\langle\cdot, \cdot\rangle_{\mathcal{A}}$ on $\mathcal{H}_{p, q}^{\theta}$, as in [175], which is used to show that $\mathcal{H}_{p, q}^{\theta}$ is a projective module. Connections are required to be compatible with the metric,

$$
\delta_{j}\langle\xi, \eta\rangle_{\mathcal{A}}=\left\langle\nabla_{j} \xi, \eta\right\rangle_{\mathcal{A}}+\left\langle\xi, \nabla_{j} \eta\right\rangle_{\mathcal{A}} .
$$

It is proved in 57 that such connections always exist. The curvature $\Omega$ has values in $\mathcal{E}=$ $\operatorname{End}_{\mathcal{A}}(\mathcal{H})$. An $\mathcal{E}$-valued inner product on $\mathcal{H}$ is given by

$$
\langle\xi, \eta\rangle_{\mathcal{E}} \zeta=\xi\langle\eta, \zeta\rangle_{\mathcal{A}},
$$


and a canonical faithful trace $\tau_{E}$ is defined as

$$
\tau_{E}\left(\langle\xi, \eta\rangle_{\mathcal{E}}\right)=\tau\left(\langle\eta, \xi\rangle_{\mathcal{A}}\right),
$$

where $\tau$ is the trace on the algebra $\mathcal{A}_{\theta}$, given by (8.8).

The Yang-Mills action is defined (cf. [88) as

$$
\tau\left(\langle\Omega, \Omega\rangle_{\mathcal{E}}\right)
$$

One seeks for minima of the Yang-Mills action among metric compatible connections (11.4). The main result is that this recovers the classical moduli spaces of Yang-Mills connections on the ordinary torus $(\underline{88})$ :

Theorem 11.1. For a choice of a pair of integers $(p, q)$ with $p+q \theta \geq 0$, the moduli space of Yang-Mills connections on the $\mathcal{A}_{\theta}$ module $\mathcal{H}_{p q}^{\theta}$ is a classical space given by the symmetric product

$$
s^{N}\left(T^{2}\right)=\left(T^{2}\right)^{N} / \Sigma_{N},
$$

where $\Sigma_{N}$ is the group of permutations in $N$-elements, for $N=\operatorname{gcd}(p, q)$.

In the matrix formulation of M-theory the basic equations to obtain periodicity of two of the basic coordinates $X_{i}$ turn out to be the

$$
U_{i} X_{j} U_{i}^{-1}=X_{j}+a \delta_{i}^{j}, \quad i=1,2,
$$

where the $U_{i}$ are unitary gauge transformations. The multiplicative commutator $U_{1} U_{2} U_{1}^{-1} U_{2}^{-1}$ is then central and in the irreducible case its scalar value $\lambda=\exp 2 \pi i \theta$ brings in the algebra of coordinates on the noncommutative torus. The $X_{j}$ are then the components of the Yang-Mills connections. The same picture emerged from the other information one has about M-theory concerning its relation with 11 dimensional supergravity and that string theory dualities can then be interpreted using Morita equivalence, relating the values of $\theta$ on an orbit of $S L_{2}(\mathbb{Z})$. Nekrasov and Schwarz [163] showed that Yang-Mills gauge theory on noncommutative $\mathbb{R}^{4}$ gives a conceptual understanding of the nonzero B-field desingularization of the moduli space of instantons obtained by perturbing the ADHM equations. In [182], Seiberg and Witten exhibited the unexpected relation between the standard gauge theory and the noncommutative one, and clarified the limit in which the entire string dynamics is described by a gauge theory on a noncommutative space. Techniques from noncommutative differential and Riemannian geometry, in the sense discussed in Section 2 were applied to string theory, for instance in 7. The role of noncommutative geometry in the context of $T$-duality was considered in very interesting recent work of Mathai and collaborators, 28], 29], 155].

Recently, in the context of the holographic description of type IIB string theory on the planewave background, Shahin M.M. Sheikh-Jabbari obtained ( $c f$. [183]) an interesting class of noncommutative spaces from the quantization of Nambu $d$-brackets. The classical Nambu brackets

$$
\left\{f_{1}, \ldots, f_{k}\right\}=\epsilon^{i_{1} \cdots i_{k}} \frac{\partial f_{1}}{\partial x^{i_{1}}} \cdots \frac{\partial f_{k}}{\partial x^{i_{k}}}
$$


of $k$ real valued functions of variables $\left(x^{1}, \ldots, x^{k}\right)$ is quantized in the even case to the expression in $2 k$ operators

$$
\frac{1}{i^{k}}\left[F_{1}, \ldots, F_{2 k}\right]=\frac{1}{i^{k}(2 k) !} \epsilon^{i_{1} \cdots i_{2 k}} F_{i_{1}} \cdots F_{i_{2 k}} .
$$

This generalizes the Poisson bracket quantization $\left\{f_{1}, f_{2}\right\} \mapsto \frac{-i}{\hbar}\left[F_{1}, F_{2}\right]$. The odd case is more subtle and it involves an additional operator related to the chirality operator $\gamma_{5}$. One sets

$$
\frac{1}{i^{k}}\left[F_{1}, \ldots, F_{2 k-1}, \gamma\right]=\frac{1}{i^{k}(2 k) !} \epsilon^{i_{1} \cdots i_{2 k}} F_{i_{1}} \cdots F_{i_{2 k-1}} \gamma,
$$

where $\gamma$ is the chirality operator in $2 k$-dimensions. For example, for $k=2$ one gets

$$
\begin{aligned}
{\left[F_{1}, F_{1}, F_{3}, \gamma\right]=} & \frac{1}{24}\left(\left[F_{1}, F_{2}\right]\left[F_{3}, \gamma\right]+\left[F_{3}, \gamma\right]\left[F_{1}, F_{2}\right]\right. \\
& -\left(\left[F_{1}, F_{3}\right]\left[F_{2}, \gamma\right]+\left[F_{2}, \gamma\right]\left[F_{1}, F_{3}\right]\right) \\
& \left.+\left[F_{2}, F_{3}\right]\left[F_{1}, \gamma\right]+\left[F_{1}, \gamma\right]\left[F_{2}, F_{3}\right]\right) .
\end{aligned}
$$

If one describes the ordinary $d$-dimensional sphere of radius $R$ by the equation

$$
\sum_{i=1}^{d+1}\left(x^{i}\right)^{2}=R^{2}
$$

the coordinates satisfy

$$
\left\{x^{i_{1}}, \ldots, x^{i_{d}}\right\}=R^{d-1} \epsilon^{i_{1} \cdots i_{d+1}} x^{i_{d+1}} .
$$

The equation (11.10) and (11.11) are then replaced by their quantized version, using the quantization of the Nambu bracket and the introduction of a quantization parameter $\ell$. This defines algebras generated by unitaries $X^{i}$ subject to the relations given by the quantization of (11.10) and (11.11). Matrix representations of these algebra correspond to certain fuzzy spheres. It would be interesting to study the general structure of these noncommutative spaces from the point of view of the various steps introduced in Section $2 . c f$. also the discussion in Section 19

\section{Groupoids And the IndeX TheOREM}

Since the construction of the $C^{*}$-algebra of foliations based on the holonomy groupoid (section 5), groupoids have played a major role in noncommutative geometry. In fact the original construction of matrix mechanics by Heisenberg (section 3) is exactly that of the convolution algebra of the groupoid of transitions imposed by experimental results. The convolution algebra of groupoids can be defined in the context of von-Neumann algebras and of $C^{*}$ algebras ( $c f$. [52] and [173]). It is particularly simple and canonical in the context of smooth groupoids ( $c f$. [54 section II.5). One virtue of the general construction is that it provides a geometric mental picture of complicated analytical constructions. The prototype example is given by the tangent groupoid of a manifold ( $c f$. 54 section II.5). It is obtained by blowing up the diagonal in the square $V \times V$ of the manifold and as a set is given by

$$
\left.\left.G_{V}=V \times V \times\right] 0,1\right] \cup T V
$$


where $T V$ is the (total space of the) tangent bundle of $V$. A tangent vector $X \in T_{x}(V)$ appears as the limit of nearby triples $\left(x_{1}, x_{2}, \varepsilon\right)$ provided in any chart the ratios $\left(x_{1}-x_{2}\right) / \varepsilon$ converge to $X$. When $\varepsilon \rightarrow 0$ the Heisenberg (matrix) law of composition :

$$
\left(x_{1}, x_{2}, \varepsilon\right) \circ\left(x_{2}, x_{3}, \varepsilon\right)=\left(x_{1}, x_{3}, \varepsilon\right)
$$

converges to the addition of tangent vectors, so that $G_{V}$ becomes a smooth groupoid. The functoriality of the construction $G \rightarrow C^{*}(G)$ of the convolution algebra for smooth groupoids $G$ is then enough to define the Atiyah-Singer analytic index of pseudo-differential operators. It is simply given by the map in K-theory for the exact sequence of $C^{*}$-algebras associated to the geometric sequence

$$
V \times V \times] 0,1] \rightarrow G_{V} \supset T V
$$

where $T V$ is viewed as a closed subgroupoid of $G_{V}$. The corresponding exact sequence of $C^{*}$-algebras can be written as

$$
\left.\left.0 \rightarrow C_{0}(] 0,1\right]\right) \times \mathcal{K} \rightarrow C^{*}\left(G_{V}\right) \rightarrow C_{0}\left(T^{*} V\right) \rightarrow 0
$$

and is a geometric form of the extension of pseudo-differential operators. By construction the algebra $\left.\left.C_{0}(] 0,1\right]\right)$ is contractible and the same holds for the tensor product $\left.\left.C_{0}(] 0,1\right]\right) \times \mathcal{K}$ by the algebra $\mathcal{K}$ of compact operators. This shows that the restriction map $C^{*}\left(G_{V}\right) \rightarrow C_{0}\left(T^{*} V\right)$ is an isomorphism in K-theory :

$$
K_{0}\left(C_{0}\left(T^{*} V\right)\right) \sim K_{0}\left(C^{*}\left(G_{V}\right)\right)
$$

Since the K-theory of $\mathcal{K}$ is $\mathbb{Z}$ for $K_{0}$, one gets the analytic index by the evaluation map

$$
C^{*}\left(G_{V}\right) \rightarrow \mathcal{K}, \quad K_{0}\left(C^{*}\left(G_{V}\right)\right) \rightarrow K_{0}(\mathcal{K})=\mathbb{Z}
$$

composed with the isomorphism (12.1). Using the Thom isomorphism yields a geometric proof ( $c f$. [54]) of the Atiyah-Singer index theorem, where all the analysis has been taken care of once and for all by the functor $G \rightarrow C^{*}(G)$.

This paradigm for a geometric set-up of the index theorem has been successfully extended to manifolds with singularities ( $c f$. [160, 161] and references there) and to manifolds with boundary [1].

\section{Riemannian MANiFOLDS, CONICAL SINGUlaRities}

A main property of the homotopy type of a compact oriented manifold is that it satisfies Poincaré duality not just in ordinary homology but also in $K$-homology. In fact, while Poincaré duality in ordinary homology is not sufficient to describe homotopy type of manifolds (cf. [159]), Sullivan proved ( $c f$. [193]) that for simply connected PL manifolds of dimension at least 5 , ignoring 2-torsion, the same property in $K O$-homology suffices and the Chern character of the $K O$-homology fundamental class carries all the rational information on the Pontrjagin classes.

For an ordinary manifold the choice of the fundamental cycle in $K$-homology is a refinement of the choice of orientation of the manifold and, in its simplest form, it is a choice of Spinstructure. Of course the role of a spin structure is to allow for the construction of the corresponding Dirac operator which gives a corresponding Fredholm representation of the algebra of smooth functions. The choice of a square root involved in the Dirac operator $D$ corresponds to a choice of $K$-orientation. 
$K$-homology admits a fairly simple definition in terms of Hilbert spaces and Fredholm representations of algebras. In fact, we have the following notion of Fredholm module (cf. [54]):

Definition 13.1. Let $\mathcal{A}$ be an algebra, an odd Fredholm module over $\mathcal{A}$ is given by:

(1) a representation of $\mathcal{A}$ in a Hilbert space $\mathcal{H}$.

(2) an operator $F=F^{*}, F^{2}=1$, on $\mathcal{H}$ such that

$[F, a]$ is a compact operator for any $a \in \mathcal{A}$.

An even Fredholm module is given by an odd Fredholm module $(\mathcal{H}, F)$ together with a $\mathbb{Z} / 2$ grading $\gamma, \gamma=\gamma^{*}, \gamma^{2}=1$ of the Hilbert space $\mathcal{H}$ satisfying:

(1) $\gamma a=a \gamma$, for all $a \in \mathcal{A}$

(2) $\gamma F=-F \gamma$.

This definition is derived from Atiyah's definition [12] of abstract elliptic operators, and agrees with Kasparov's definition [123] for the cycles in $K$-homology, $K K(A, \mathbb{C})$, when $A$ is a $C^{*}$-algebra.

The notion of Fredholm module can be illustrated by the following examples (cf. [54]).

Example 13.2. If $X$ is a manifold, an elliptic operator on $X$ can be twisted with vector bundles, so as to give rise to an index map Ind : $K_{0}(C(X)) \rightarrow \mathbb{Z}$. If $P$ is an elliptic operator (the symbol is invertible) and a pseudodifferential operator of order zero, $P: L^{2}\left(X, E_{+}\right) \rightarrow$ $L^{2}\left(X, E_{-}\right)$, then there exists a parametrix $Q$ for $P$. This is also an operator of order zero, and a quasi-inverse for $P$, in the sense that it is an inverse at the symbol level, namely $P Q-I$ and $Q P-I$ are compact operators. Consider then the operator

$$
F=\left(\begin{array}{cc}
0 & Q \\
P & 0
\end{array}\right)
$$

on $\mathcal{H}=L^{2}\left(X, E_{+}\right) \oplus L^{2}\left(X, E_{-}\right)$. The algebra $C(X)$ acts on $\mathcal{H}$ and $[F, f]$ is a compact operator for all $f \in C(X)$. Since $F^{2}-I$ is compact, it is possible to add to $\mathcal{H}$ a finite dimensional space to obtain $F^{2}=I$. Notice that the functions of $C(X)$ act differently on this modified space. In particular the function $f \equiv 1$ no longer acts as the identity: one recovers the index of $P$ this way.

Example 13.3. Let $\Gamma=\mathbb{Z} * \mathbb{Z}$ a free group, and let $\mathcal{A}=\mathbb{C} \Gamma$. Let $\mathcal{T}$ be the tree of $\Gamma$ with $\mathcal{T}^{0}$ the set of vertices and $\mathcal{T}^{1}$ the set of edges. Fix an origin $x_{0}$ in $\mathcal{T}^{0}$. For any vertex $v \in \mathcal{T}^{0}$ there exists a unique path connecting it to the origin $x_{0}$. This defines a bijection $\phi: \mathcal{T}^{0} \backslash\left\{x_{0}\right\} \rightarrow \mathcal{T}^{1}$ that assigns $v \mapsto \phi(v)$ with $\phi(v)$ this unique edge. Let $U_{\phi}$ be the unitary operator implementing $\phi$, and consider the operator

$$
F=\left(\begin{array}{cc}
0 & U_{\phi} \\
U_{\phi}^{*} & 0
\end{array}\right)
$$

acting on $\mathcal{H}=\ell^{2}\left(\mathcal{T}^{0}\right) \oplus \ell^{2}\left(\mathcal{T}^{1}\right) \oplus \mathbb{C}$. By construction $\Gamma$ acts naturally on $\mathcal{T}_{j}$ which gives a corresponding action of $\mathcal{A}$ in $\mathcal{H}$. The pair $(\mathcal{H}, F)$ is a Fredholm module over $\mathcal{A}$.

Example 13.4. On $S^{1} \simeq \mathbb{P}^{1}(\mathbb{R})$, consider the algebra of functions $\mathrm{C}\left(\mathbb{P}^{1}(\mathbb{R})\right)$, acting on the Hilbert space $L^{2}(\mathbb{R})$, as multiplication operators $(f \xi)(s)=f(s) \xi(s)$. Let $F$ be the Hilbert transform

$$
(F \xi)(s)=\frac{1}{\pi i} \int \frac{f(t)}{s-t} d t
$$


This multiplies by +1 the positive Fourier modes and by -1 the negative Fourier modes. A function $f \in \mathrm{C}\left(\mathbb{P}^{1}(\mathbb{R})\right)$ has the property that $[F, f]$ is of finite rank if and only if $f$ is a rational function $f(s)=P(s) / Q(s)$. This is Kronecker's characterization of rational functions.

Besides the $K$-homology class, specified by a Fredholm module, one also wants to generalize to the noncommutative setting the infinitesimal line element $d s$ of a Riemannian manifold. In ordinary Riemannian geometry one deals rather with the $d s^{2}$ given by the usual local expression $g_{\mu \nu} d x^{\mu} d x^{\nu}$. However, in order to extend the notion of metric space to the noncommutative setting it is more natural to deal with $d s$, for which the ansatz is

$$
d s=\times \times,
$$

where the right hand side has the meaning usually attributed to it in physics, namely the Fermion propagator

$$
\ltimes \times=D^{-1},
$$

where $D$ is the Dirac operator. In other words, the presence of a spin (or $\operatorname{spin}^{c}$ ) structure makes it possible to extract the square root of $d s^{2}$, using the Dirac operator as a differential square root of a Laplacian.

This prescription recovers the usual geodesic distance on a Riemannian manifold, by the following result ( $c f .[59])$.

Lemma 13.5. On a Riemannian spin manifold the geodesic distance $d(x, y)$ between two points is computed by the formula

$$
d(x, y)=\sup \{|f(x)-f(y)| ; f \in \mathcal{A},\|[D, f]\| \leq 1\}
$$

where $D$ is the Dirac operator, $D=d s^{-1}$, and $\mathcal{A}$ is the algebra of smooth functions.

This essentially follows from the fact that the quantity $\|[D, f]\|$ can be identified with the Lipschitz norm of the function $f$,

$$
\|[D, f]\|=\operatorname{ess} \sup _{x \in M}\left\|(\nabla f)_{x}\right\|=\sup _{x \neq y} \frac{|f(x)-f(y)|}{d(x, y)} .
$$

Notice that, if $d s$ has the dimension of a length $L$, then $D$ has dimension $L^{-1}$ and the above expression for $d(x, y)$ also has the dimension of a length. On a Riemannian spin manifold $X$, the condition $\|[D, f]\| \leq 1$, for $D$ the Dirac operator, is equivalent to the condition that $f$ is a Lipschitz function with Lipschitz constant $c \leq 1$.

The advantage of the definition (13.1), (13.2) of the line element is that it is of a spectral and operator theoretic nature, hence it extends to the noncommutative setting.

The structure that combines the $K$-homology fundamental cycle with the spectral definition of the line element $d s$ is the notion of spectral triple $(\mathcal{A}, \mathcal{H}, D)(c f$. [63, [81]).

Definition 13.6. A (compact) noncommutative geometry is a triple

$$
(\mathcal{A}, \mathcal{H}, D)
$$

where $\mathcal{A}$ is a unital algebra represented concretely as an algebra of bounded operators on the Hilbert space $\mathcal{H}$. The unbounded operator $D$ is the inverse of the line element

$$
d s=1 / D \text {. }
$$

Such a triple $(\mathcal{A}, \mathcal{H}, D)$ is requires to satisfy the properties: 
(1) $[D, a]$ is bounded for any $a \in A^{\infty}$, a dense subalgebra of the $C^{*}$-algebra $A=\overline{\mathcal{A}}$.

(2) $D=D^{*}$ and $(D+\lambda)^{-1}$ is a compact operator, for all $\lambda \notin \mathbb{R}$.

We say that a spectral triple $(\mathcal{A}, \mathcal{H}, D)$ is even if the Hilbert space $\mathcal{H}$ has a $\mathbb{Z} / 2$-grading by an operator $\gamma$ satisfying

$$
\gamma=\gamma^{*}, \quad \gamma^{2}=1, \quad \gamma D=-D \gamma, \quad \gamma a=a \gamma \quad \forall a \in A
$$

This definition is entirely spectral. The elements of the algebra (in general noncommutative) are operators and the line element is also an operator. The polar decomposition $D=|D| F$ recovers the Fredholm module $F$ defining the fundamental class in $K$-homology. The formula for the geodesic distance extends to this context as follows.

Definition 13.7. Let $\varphi_{i}: A \rightarrow \mathbb{C}$, for $i=1,2$, be states on $A$, i.e. normalized positive linear functionals on $A$ with $\varphi_{i}(1)=1$ and $\varphi_{i}\left(a^{*} a\right) \geq 0$ for all $a \in A$. Then the distance between them is given by the formula

$$
d\left(\varphi_{1}, \varphi_{2}\right)=\sup \left\{\left|\varphi_{1}(a)-\varphi_{2}(a)\right| ; a \in A,\|[D, a]\| \leq 1\right\} .
$$

A spectral triple $(\mathcal{A}, \mathcal{H}, D)$ is of metric dimension $p$, or $p$-summable, if $|D|^{-1}$ is an infinitesimal of order $1 / p$ (i.e. $|D|^{-p}$ is an infinitesimal of order one). Here $p<\infty$ is a positive real number. A spectral triple $(\mathcal{A}, \mathcal{H}, D)$ is $\theta$-summable if $\operatorname{Tr}\left(e^{-t D^{2}}\right)<\infty$ for all $t>0$. The latter case corresponds to an infinite dimensional geometry.

Spectral triples also provide a more refined notion of dimension besides the metric dimension (summability). It is given by the dimension spectrum, which is not a number but a subset of the complex plane.

More precisely, let $(\mathcal{A}, \mathcal{H}, D)$ be a spectral triple satisfying the regularity hypothesis

$$
a \text { and }[D, a] \in \cap_{k} \operatorname{Dom}\left(\delta^{k}\right), \forall a \in A^{\infty},
$$

where $\delta$ is the derivation $\delta(T)=[|D|, T]$, for any operator $T$. Let $\mathcal{B}$ denote the algebra generated by $\delta^{k}(a)$ and $\delta^{k}([D, a])$. The dimension spectrum of the triple $(\mathcal{A}, \mathcal{H}, D)$ is the subset $\Sigma \subset \mathbb{C}$ consisting of all the singularities of the analytic functions $\zeta_{b}(z)$ obtained by continuation of

$$
\zeta_{b}(z)=\operatorname{Tr}\left(b|D|^{-z}\right), \quad \Re(z)>p, \quad b \in \mathcal{B} .
$$

Example 13.8. Let $M$ be a smooth compact Riemannian spin manifold, and $(\mathcal{A}, \mathcal{H}, D)$ is the corresponding spectral triple given by the algebra of smooth functions, the space of spinors, and the Dirac operator. Then the metric dimension agrees with the usual dimension $n$ of $M$. The dimension spectrum of $M$ is the set $\{0,1, \ldots, n\}$, where $n=\operatorname{dim} M$, and it is simple. (Multiplicities appear for singular manifolds.)

It is interesting in the case of an ordinary Riemannian manifold $M$ to see the meaning of the points in the dimension spectrum that are smaller than $n=\operatorname{dim} M$. These are dimensions in which the space "manifests itself nontrivially" with some interesting geometry.

For instance, at the point $n=\operatorname{dim} M$ of the dimension spectrum one can recover the volume form of the Riemannian metric by the equality (valid up to a normalization constant $c f$. [54])

$$
f f|d s|^{n}=\int_{M_{n}} f \sqrt{g} d^{n} x
$$


where the integral $f T$ is given ( $c f$. [54]) by the Dixmier trace ( $c f$. 97]) generalizing the Wodzicki residue of pseudodifferential operators ( $c f$. [202]).

One can also consider integration $f d s^{k}$ in any other dimension in the dimension spectrum, with $d s=D^{-1}$ the line element. In the case of a Riemannian manifold one finds other important curvature expressions. For instance, if $M$ is a manifold of $\operatorname{dimension} \operatorname{dim} M=4$, when one considers integration in dimension 2 one finds the Einstein-Hilbert action. In fact, a direct computation yields the following result ( $c f$. 124] [122]):

Proposition 13.9. Let $d v=\sqrt{g} d^{4} x$ denote the volume form, $d s=D^{-1}$ the length element, i.e. the inverse of the Dirac operator, and $r$ the scalar curvature. We obtain:

$$
f d s^{2}=\frac{-1}{48 \pi^{2}} \int_{M_{4}} r d v
$$

In general, one obtains the scalar curvature of an $n$-dimensional manifold from the integral $f d s^{n-2}$.

Many interesting examples of spectral triples just satisfy the conditions stated in Definition 13.6. However, there are significant case where more refined properties of manifolds carry over to the noncommutative case, such as the presence of a real structure (which makes it possible to distinguish between $K$-homology and $K O$-homology) and the "order one condition" for the Dirac operator. These properties are described as follows ( $c f .61$ and [62]).

Definition 13.10. A real structure on an $n$-dimensional spectral triple $(\mathcal{A}, \mathcal{H}, D)$ is an antilinear isometry $J: \mathcal{H} \rightarrow \mathcal{H}$, with the property that

$$
J^{2}=\varepsilon, \quad J D=\varepsilon^{\prime} D J, \text { and } J \gamma=\varepsilon^{\prime \prime} \gamma J \text { (even case). }
$$

The numbers $\varepsilon, \varepsilon^{\prime}, \varepsilon^{\prime \prime} \in\{-1,1\}$ are a function of $n \bmod 8$ given by

\begin{tabular}{|c|rrrrrrrr|}
\hline $\mathbf{n}$ & 0 & 1 & 2 & 3 & 4 & 5 & 6 & 7 \\
\hline \hline$\varepsilon$ & 1 & 1 & -1 & -1 & -1 & -1 & 1 & 1 \\
$\varepsilon^{\prime}$ & 1 & -1 & 1 & 1 & 1 & -1 & 1 & 1 \\
$\varepsilon^{\prime \prime}$ & 1 & & -1 & & 1 & & -1 & \\
\hline
\end{tabular}

Moreover, the action of $\mathcal{A}$ satisfies the commutation rule

$$
\left[a, b^{0}\right]=0 \quad \forall a, b \in \mathcal{A},
$$

where

$$
b^{0}=J b^{*} J^{-1} \quad \forall b \in \mathcal{A},
$$

and the operator $D$ satisfies

$$
\left[[D, a], b^{0}\right]=0 \quad \forall a, b \in \mathcal{A} .
$$

The anti-linear isometry $J$ is given, in ordinary Riemannian geometry, by the charge conjugation operator acting on spinors. In the noncommutative case, this is replaced by the Tomita antilinear conjugation operator ( $c f .[195]$ ).

In 61] and 103. Theorem 11.2, necessary and sufficient conditions are given that a spectral triple $(\mathcal{A}, \mathcal{H}, D)$ (with real structure $J$ ) should fulfill in order to come from an ordinary compact Riemannian spin manifold:

(1) $d s=D^{-1}$ is an infinitesimal of order $1 / n$. 
(2) There is a real structure in the sense of Definition 13.10.

(3) The commutation relation (13.15) holds (this is $[[D, a], b]=0$, for all $a, b \in \mathcal{A}$, when $\mathcal{A}$ is commutative).

(4) The regularity hypothesis of (13.8) holds: $a$ and $[D, a]$ are in $\cap_{k} \operatorname{Dom}\left(\delta^{k}\right)$ for all $a \in \mathcal{A}_{\infty}$.

(5) There exists a Hochschild cycle $c \in Z_{n}\left(\mathcal{A}_{\infty}, \mathcal{A}_{\infty}\right)$ such that its representation $\pi(c)$ on $\mathcal{H}$ induced by

$$
\pi\left(a^{0} \otimes \cdots \otimes a^{n}\right)=a^{0}\left[D, a^{1}\right] \cdots\left[D, a^{n}\right]
$$

satisfies $\pi(c)=\gamma$, for $\gamma$ as in (13.6), in the even case, and $\pi(c)=1$ in the odd case.

(6) The space $\mathcal{H}^{\infty}=\cap_{k} \operatorname{Dom}\left(D^{k}\right)$ is a finite projective $\mathcal{A}$-module, endowed with a $\mathcal{A}$ valued inner product $\langle\xi, \eta\rangle_{\mathcal{A}}$ defined by

$$
\langle a \xi, \eta\rangle=f_{a}\langle\xi, \eta\rangle_{\mathcal{A}} d s^{n}
$$

(7) The intersection form

$$
K_{*}(\mathcal{A}) \times K_{*}(\mathcal{A}) \rightarrow \mathbb{Z}
$$

obtained from the Fredholm index of $D$ with coefficients in $K_{*}\left(\mathcal{A} \otimes \mathcal{A}^{0}\right)$ is invertible.

When $\mathcal{A}$ is commutative, the above conditions characterize a smooth Riemannian manifold $M$, with $\mathcal{A}_{\infty}=\mathcal{C}^{\infty}(M)$ (we refer to [103] for the precise statement). However, the conditions can be stated without any commutativity assumption on $\mathcal{A}$. They are satisfied, for instance, by the isospectral deformations of [72, which we discuss in Section 18, Another very significant noncommutative example is the standard model of elementary particles ( $c f$. 61]), which we discuss in Section 17

Another example of spectral triple associated to a classical space, which is not classically a smooth manifold, is the case of manifolds with singularities. In particular, one can consider the case of an isolated conical singularity. This case was studied by Lescure 141.

Let $X$ be a manifold with an isolated conical singularity. The cone point $c \in X$ has the property that there is a neighborhood $U$ of $c$ in $X$, such that $U \backslash\{c\}$ is of the form $(0,1] \times N$, with $N$ a smooth compact manifold, and metric $\left.g\right|_{U}=d r^{2}+r^{2} g_{N}$, where $g_{N}$ is the metric on $N$.

A natural class of differential operators on manifolds with isolated conical singularities is given by the elliptic operators of Fuchs type, acting on sections of a bundle $E$. These are operators whose restriction over $(0,1] \times N$ takes the form

$$
r^{-\nu} \sum_{k=0}^{d} a_{k}(r)\left(-r \partial_{r}\right)^{k},
$$

for $\nu \in \mathbb{R}$ and $a_{k} \in C^{\infty}\left([0,1], \operatorname{Diff}^{d-k}\left(N,\left.E\right|_{N}\right)\right)$, which are elliptic with symbol $\sigma_{M}(D)=$ $\sum_{k=0}^{d} a_{k}(0) z^{k}$ that is an elliptic family parameterized by $\operatorname{Im}(z)$. In particular, operators of Dirac type are elliptic of Fuchs type. For such an operator $D$, which is of first order and symmetric, results of Chou [48], Brüning, Seeley [33] and Lesch [140] show that its self-adjoint extension has discrete spectrum, with $(n+1)$-summable resolvent, for $\operatorname{dim} X=n$. 
The algebra that is used in the construction of the spectral triple is $\mathcal{A}=C_{c}^{\infty}(X) \oplus \mathbb{C}$, the algebra of functions that are smooth on $X \backslash\{c\}$ and constant near the singularity. The Hilbert space on which $D$ acts is chosen from a family of weighted Sobolev spaces. Roughly, one defines weighted Sobolev spaces that look like the standard Sobolev space on the smooth part and on the cone are defined locally by norms

$$
\|f\|_{s, \gamma}^{2}=\int_{\mathbb{R}_{+}^{*} \times \mathbb{R}^{m-1}}\left(1+(\log t)^{2}+\xi^{2}\right)^{s}\left|\left(r^{-\gamma+1 / 2} f\right)(t, \xi)\right|^{2} \frac{d t}{t} d \xi,
$$

where $\hat{f}$ denotes Fourier transform on the group $\mathbb{R}_{+}^{*} \times \mathbb{R}^{m-1}$.

Then one obtains the following result (cf. [141])

Theorem 13.11. The data $(\mathcal{A}, \mathcal{H}, D)$ given above define a spectral triple. In particular, the zeta functions $\operatorname{Tr}\left(a|D|^{-z}\right)$ admit analytic continuation to $\mathbb{C} \backslash \Sigma$, where the dimension spectrum is of the form

$$
\Sigma=\{\operatorname{dim} X-k, k \in \mathbb{N}\}
$$

with multiplicities $\leq 2$.

The analysis of the zeta functions uses the heat kernel

$$
\operatorname{Tr}\left(|D|^{-z}\right)=\frac{1}{\Gamma(z / 2)} \int_{0}^{\infty} t^{z / 2-1} \operatorname{Tr}\left(e^{-t D^{2}}\right) d t
$$

for which one can rely on the results of [48] and [140]. The case of $\operatorname{Tr}\left(a|D|^{-z}\right)$ of the form $\operatorname{Tr}\left(Q|D|^{-z}\right)$ with $Q \in \Psi_{c}^{\ell}(E)$, is treated by splitting $Q|D|^{-z}$ as a sum of a contribution from the smooth part and one from the singularity.

The Chern character for this spectral triple gives a map

$$
C h: K_{*}(X) \rightarrow H_{*}(X, \mathbb{C})
$$

where we have $K^{*}(X) \cong K_{*}(\mathcal{A})$ and $H_{*}(X, \mathbb{C}) \cong P H C^{*}(\mathcal{A})$, the periodic cyclic homology of the algebra $\mathcal{A}$.

The cocycles $\varphi_{n}$ in the $(b, B)$-bicomplex for the algebra $\mathcal{A}$ have also been computed explicitly and are of the form

$$
\varphi_{n}\left(a_{0}, \ldots, a_{n}\right)=\nu_{n} \int_{X} a_{0} d a_{1} \wedge \cdots \wedge d a_{n} \wedge \hat{A}(X) \wedge C h(E)
$$

for $n \geq 1$, while for $n=0, \lambda \in \mathbb{C}, \varphi_{0}(a+\lambda)=\int_{X} a \hat{A}(X) \wedge C h(E)+\lambda \operatorname{Ind}\left(D_{+}\right)$.

\section{CAntor SETS AND FRACTALS}

An important class of $C^{*}$-algebras are those obtained as direct limits of a sequence of finite dimensional subalgebras and embeddings. These are called approximately finite dimensional, or simply AF-algebras.

An $\mathrm{AF}$ algebra $\mathcal{A}$ is determined by a diagram of finite dimensional algebras and inclusions, its Bratteli diagram [31, and from the diagram itself it is possible to read a lot of the structure of the algebra, for instance its ideal structure. Some simple examples of algebras that belong to this class are: 
Example 14.1. An example of a commutative $A F$ is the algebra of complex valued continuous functions on a Cantor set, where a Bratteli diagram is determined by a decreasing family of disjoint intervals covering the Cantor set.

A non-commutative example of AF algebra is given by the algebra of the canonical anticommutation relations of quantum mechanics.

Example 14.2. Consider a real Hilbert space $\mathcal{E}$ and a linear map $\mathcal{E} \rightarrow \mathcal{B}(\mathcal{H}), f \mapsto T_{f}$, to bounded operators in a Hilbert space $\mathcal{H}$, satisfying

$$
\begin{gathered}
T_{f} T_{g}+T_{g} T_{f}=0 \\
T_{f}^{*} T_{g}+T_{g} T_{f}^{*}=\langle g, f\rangle I,
\end{gathered}
$$

and the algebra $\mathcal{A}$ generated by all the operators $T_{f}$ satisfying these relations.

A survey with many examples of AF algebras and their properties is given for instance in 96.

Let $\mathcal{A}$ be a commutative $\mathrm{AF} \mathrm{C}^{*}$-algebra. A commutative $\mathrm{AF}$ algebra $\mathcal{A}$ is spanned by its projections, since finite dimensional commutative algebras are generated by orthogonal projections. This condition is equivalent to the $\operatorname{spectrum} \Lambda=\operatorname{Spec}(\mathcal{A})$ of the algebra being a totally disconnected compact Hausdorff space, typically a Cantor set. Realizing such Cantor set as the intersection of a decreasing family of disjoint intervals covering $\Lambda$ also provides a Bratteli diagram for the $\mathrm{AF}$ algebra $\mathcal{A}=C(\Lambda)$.

As described in [54, in order to construct the Hilbert space $\mathcal{H}$ for a Cantor set $\Lambda \subset \mathbb{R}$, let $J_{k}$ be the collection of bounded open intervals in $\mathbb{R} \backslash \Lambda$. We denote by $L=\left\{\ell_{k}\right\}_{k \geq 1}$ the countable collection of the lengths of the intervals $J_{k}$. We can assume that the lengths are ordered

$$
\ell_{1} \geq \ell_{2} \geq \ell_{3} \geq \cdots \geq \ell_{k} \cdots>0 \text {. }
$$

We also denote by $E=\left\{x_{k, \pm}\right\}$ the set of the endpoints of the intervals $J_{k}$, with $x_{k,+}>x_{k,-}$. Consider the Hilbert space

$$
\mathcal{H}:=\ell^{2}(E)
$$

Since the endpoints of the $J_{k}$ are points of $\Lambda$, there is an action of $\mathrm{C}(\Lambda)$ on $\mathcal{H}$ given by

$$
f \cdot \xi(x)=f(x) \xi(x), \quad \forall f \in \mathrm{C}(\Lambda), \forall \xi \in \mathcal{H}, \forall x \in E .
$$

A sign operator $F$ can be obtained $(c f .54$ ) by choosing the closed subspace $\hat{\mathcal{H}} \subset \mathcal{H}$ given by

$$
\hat{\mathcal{H}}=\left\{\xi \in \mathcal{H}: \xi\left(x_{k,-}\right)=\xi\left(x_{k,+}\right), \forall k\right\} .
$$

Then $F$ has eigenspaces $\hat{\mathcal{H}}$ with eigenvalue +1 and $\hat{\mathcal{H}}^{\perp}$ with eigenvalue -1 , so that, when restricted to the subspace $\mathcal{H}_{k}$ of coordinates $\xi\left(x_{k,+}\right)$ and $\xi\left(x_{k,-}\right)$, the sign $F$ is given by

$$
\left.F\right|_{\mathcal{H}_{k}}=\left(\begin{array}{cc}
0 & 1 \\
1 & 0
\end{array}\right)
$$

Finally, a Dirac operator $D=|D| F$ is obtained as

$$
\left.D\right|_{\mathcal{H}_{k}}\left(\begin{array}{c}
\xi\left(x_{k,+}\right) \\
\xi\left(x_{k,-}\right)
\end{array}\right)=\ell_{k}^{-1} \cdot\left(\begin{array}{c}
\xi\left(x_{k,-}\right) \\
\xi\left(x_{k,+}\right)
\end{array}\right) .
$$

We then obtain the following result. 


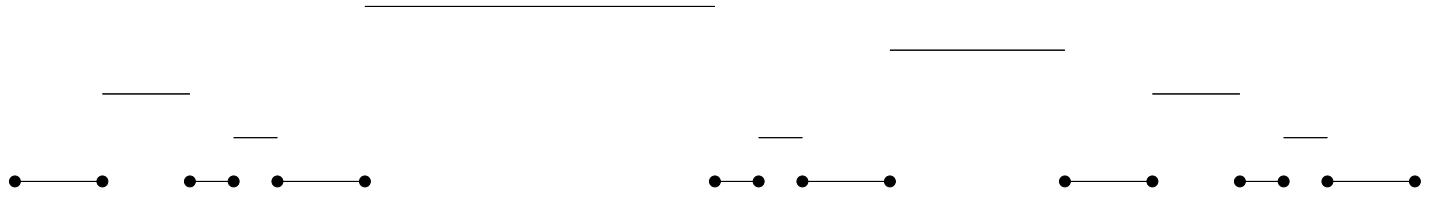

Figure 13. The Fibonacci Cantor set.

Proposition 14.3. Let $\Lambda \subset \mathbb{R}$ be a Cantor set. Let $\mathcal{A}_{\infty} \subset C(\Lambda)$ be the dense subalgebra of locally constant functions on the Cantor set. Then the data $(\mathcal{A}, \mathcal{H}, D)$ form a spectral triple, with $\mathcal{H}$ as in (14.2), the action (14.3), and $D$ as in 14.5). The zeta function satisfies

$$
\operatorname{Tr}\left(|D|^{-s}\right)=2 \zeta_{L}(s)
$$

where $\zeta_{L}(s)$ is the geometric zeta function of $L=\left\{\ell_{k}\right\}_{k \geq 1}$, defined as

$$
\zeta_{L}(s):=\sum_{k} \ell_{k}^{s}
$$

These zeta functions are related to the theory of Dirichlet series and to other arithmetic zeta functions, and also to Ruelle's dynamical zeta functions ( $c f$. [137]).

For example, for the classical middle-third Cantor set, we have set of lengths $\ell_{k}=3^{-k}$ and multiplicities $m_{k}=2^{k-1}$, for $k \geq 1$, so that we obtain

$$
\operatorname{Tr}\left(|D|^{-s}\right)=2 \zeta_{L}(s)=\sum_{k \geq 1} 2^{k} 3^{-s k}=\frac{2 \cdot 3^{-s}}{1-2 \cdot 3^{-s}} .
$$

This shows that the dimension spectrum of the spectral triple of a Cantor set has points off the real line. In fact, the set of poles of (14.7) is

$$
\left\{\frac{\log 2}{\log 3}+\frac{2 \pi i n}{\log 3}\right\}_{n \in \mathbb{Z}} .
$$

In this case the dimension spectrum lies on a vertical line and it intersects the real axis in the point $D=\frac{\log 2}{\log 3}$ which is the Hausdoff dimension of the ternary Cantor set. The same is true for other Cantor sets, as long as the self-similarity is given by a unique contraction (in the ternary case the original interval is replaced by two intervals of lengths scaled by $1 / 3$ ). If one considers slightly more complicated fractals in $\mathbb{R}$, where the self-similarity requires more than one scaling map, the dimension spectrum may be correspondingly more complicated. This can be seen in the case of the Fibonacci Cantor set, for instance ( $c f$. [137]).

The Fibonacci Cantor set $\Lambda$ is obtained from the interval $I=[0,4]$ by successively removing $F_{n+1}$ open intervals $J_{n, j}$ of lengths $\ell_{n}=1 / 2^{n}$ according to the rule of Figure 13. We can associate to this Cantor set the commutative AF algebra $\mathcal{A}=C(\Lambda)$.

To obtain the Hilbert space we consider again the set $E$ of endpoints $x_{n, j, \pm}$ of the intervals $J_{n, j}$ and we take $\mathcal{H}=\ell^{2}(E)$. We define the Dirac operator as in the previous case, and we again consider the dense involutive subalgebra $\mathcal{A}_{\infty}$ of locally constant functions. 
The data $(\mathcal{A}, \mathcal{H}, D)$ give a spectral triple. The Dirac operator is related to the geometric zeta function of the Fibonacci Cantor set by

$$
\operatorname{Tr}\left(|D|^{-s}\right)=2 \zeta_{F}(s)=\frac{2}{1-2^{-s}-4^{-s}},
$$

where the geometric zeta function is $\zeta_{F}(s)=\sum_{n} F_{n+1} 2^{-n s}$, with $F_{n}$ the Fibonacci numbers. A simple argument shows that the dimension spectrum is given by the set

$$
\Sigma=\left\{\frac{\log \phi}{\log 2}+\frac{2 \pi i n}{\log 2}\right\}_{n \in \mathbb{Z}} \cup\left\{-\frac{\log \phi}{\log 2}+\frac{2 \pi i(n+1 / 2)}{\log 2}\right\}_{n \in \mathbb{Z}},
$$

where $\phi=\frac{1+\sqrt{5}}{2}$ is the golden ratio.

Recent results on the noncommutative geometry of fractals and Cantor sets and spectral triple constructions for AF algebras can be found in [6], 108, [109]. The construction in [6] is in fact a spectral triple for the dual group of the Cantor set seen as the product of countably many copies of the group $\mathbb{Z} / 2$. The recent work [49] shows that it is easy to describe a compact metric space exactly (i.e. recovering the metric) via a spectral triple, which is a sum of two-dimensional modules, but spectral triples carry much more information than just the one regarding the metric.

\section{Spaces of Dimension $z$ And DimReg}

In perturbative quantum field theory, one computes expectation values of observables via a formal series, where the terms are parameterized by Feynman graphs and reduce to ordinary finite dimensional integrals in momentum space of expressions assigned to the graphs by the Feynman rules. These expressions typically produce divergent integrals. For example, in the example of the scalar $\phi^{3}$ theory in dimension $D=4$ or $D=4+2 \mathbb{N}$, one encounters a divergence already in the simplest one loop diagram, with corresponding integral (in Euclidean signature)

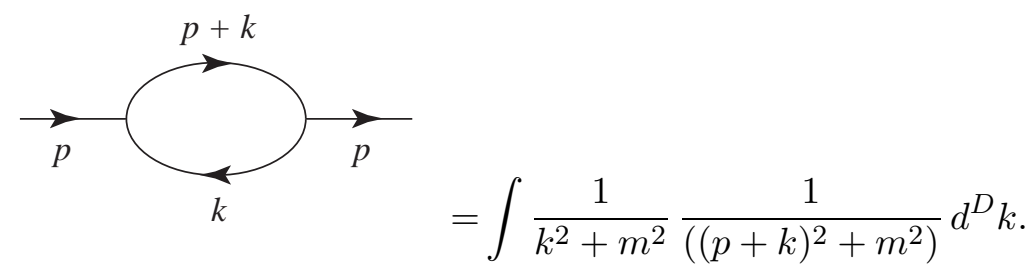

One needs therefore a regularization procedure for these divergent integrals. The regularization most commonly adopted in quantum field theory computation is "Dimensional Regularization (DimReg) and Minimal Subtraction (MS)". The method was introduced in the '70s in [23] and [118] and it has the advantage of preserving basic symmetries.

The regularization procedure of DimReg is essentially based on the use of the formula

$$
\int e^{-\lambda k^{2}} d^{d} k=\pi^{d / 2} \lambda^{-d / 2}
$$

to define the meaning of the integral in $d=(D-z)$-dimensions, for $z \in \mathbb{C}$ in a neighborhood of zero. For instance, in the case of (15.1), the procedure of dimensional regularization yields 
the result

$$
\pi^{(D-z) / 2} \Gamma\left(\frac{4-D+z}{2}\right) \int_{0}^{1}\left(\left(x-x^{2}\right) p^{2}+m^{2}\right)^{\frac{D-z-4}{2}} d x .
$$

In the recent survey 142, Yuri Manin refers to DimReg as "dimensions in search of a space" ${ }^{1}$. Indeed, in the usual approach in perturbative quantum field theory, the dimensional regularization procedure is just regarded as a formal rule of analytic continuation of formal (divergent) expressions in integral dimensions $D$ to complex values of the variable $D$.

However, using noncommutative geometry, it is possible to construct actual spaces (in the sense of noncommutative Riemannian geometry) $X_{z}$ whose dimension (in the sense of dimension spectrum) is a point $z \in \mathbb{C}(c f .[76])$.

It is well known in the physics literature that there are problems related to using dimensionl regularization in chiral theory, because of how to give a consistent prescription on how to extend the $\gamma_{5}$ (the product of the matrices $\gamma^{i}$ when $D=4$ ) to noninteger dimension $D-$ $z$. It turns out that a prescription known as Breitenlohner-Maison ([32], [50]) admits an interpretation in terms of the cup product of spectral triples, where one takes the product of the spectral triple associated to the ordinary geometry in the integer dimension $D$ by a spectral triple $X_{z}$ whose dimension spectrum is reduced to the complex number $z$ (cf. [76]). We illustrate here the construction for the case where $z \in \mathbb{R}_{+}^{*}$. The more general case of $z \in \mathbb{C}$ is more delicate.

One needs to work in a slightly modified setting for spectral triples, which is given by the type II spectral triples ( $c f$. [21], 38], 39]). In this setting the usual type I trace of operators in $\mathcal{L}(\mathcal{H})$ is replaced by the trace on a type $\mathrm{II}_{\infty}$ von-Neumann algebra.

One considers a self-adjoint operator $Y$, affiliated to a type $\mathrm{II}_{\infty}$ factor $N$, with spectral measure given by

$$
\operatorname{Tr}_{N}\left(\chi_{E}(Y)\right)=\frac{1}{2} \int_{E} d y
$$

for any interval $E \subset \mathbb{R}$, with characteristic function $\chi_{E}$. If $Y=F|Y|$ is the polar decomposition of $Y$, one sets

$$
D_{z}=\rho(z) F|Y|^{1 / z}
$$

with the complex power $|Y|^{1 / z}$ defined by the functional calculus. The normalization constant $\rho(z)$ is chosen to be

$$
\rho(z)=\pi^{-\frac{1}{2}}\left(\Gamma\left(\frac{z}{2}+1\right)\right)^{\frac{1}{z}}
$$

so that one obtains

$$
\operatorname{Tr}\left(e^{-\lambda D^{2}}\right)=\pi^{z / 2} \lambda^{-z / 2} \quad \forall \lambda \in \mathbb{R}_{+}^{*} .
$$

This gives a geometric meaning to the basic formula (15.2) of DimReg. The algebra $\mathcal{A}$ of the spectral triple $X_{z}$ can be made to contain any operator $a$ such that $\left[D_{z}, a\right]$ is bounded and both $a$ and $\left[D_{z}, a\right]$ are smooth for the "geodesic flow"

$$
T \mapsto e^{i t\left|D_{z}\right|} T e^{-i t\left|D_{z}\right|} .
$$

\footnotetext{
${ }^{1}$ Nicely reminiscent of Pirandello's play "Six characters in search of an author".
} 
The dimension spectrum of $X_{z}$ is reduced to the single point $z$, since

$$
\operatorname{Trace}_{N}^{\prime}\left(\left(D_{z}^{2}\right)^{-s / 2}\right)=\rho^{-s} \int_{1}^{\infty} u^{-s / z} d u=\rho^{-s} \frac{z}{s-z}
$$

has a single (simple) pole at $s=z$ and is absolutely convergent in the half space $\operatorname{Re}(s / z)>1$. Here Trace $_{N}^{\prime}$ denotes the trace with an infrared cutoff (i.e. integrating outside $|y|<1$ ).

\section{LOCAL ALGEBRAS IN SUPERSYMMETRIC QFT}

It is quite striking that the general framework of noncommutative geometry is suitable not only for handling finite dimensional spaces (commutative or not, of non-integer dimension etc.) but is also compatible with infinite dimensional spaces. We already saw in Section 7 that discrete groups of exponential growth naturally give rise to noncommutative spaces which are described by a $\theta$-summable spectral triple, but not by a finitely summable spectral triple. This is characteristic of an infinite dimensional space and in that case, as we saw for discrete groups, cyclic cohomology needs to be extended to entire cyclic cohomology. A very similar kind of noncommutative spaces arises from Quantum Field Theory in the supersymmetric context [54 Section IV.9. $\beta$. We briefly recall this below and then explain open questions also in the context of supersymmetric theories.

The simplest example to understand the framework is that of the free Wess-Zumino model in two dimensions, a supersymmetric free field theory in a two dimensional space-time where space is compact ([54]). Thus space is a circle $S^{1}$ and space-time is a cylinder $C=S^{1} \times \mathbb{R}$ endowed with the Lorentzian metric. The fields are given by a complex scalar bosonic field $\phi$ of mass $m$ and a spinor field $\psi$ of the same mass. The Lagrangian of the theory is of the form $\mathcal{L}=\mathcal{L}_{b}+\mathcal{L}_{f}$ where,

$$
\mathcal{L}_{b}=\frac{1}{2}\left(\left|\partial_{0} \phi\right|^{2}-\left|\partial_{1} \phi\right|^{2}-m^{2}|\phi|^{2}\right)
$$

and for the fermions,

$$
\mathcal{L}_{f}=i \bar{\psi} \gamma^{\mu} \partial_{\mu} \psi-m \bar{\psi} \psi
$$

where the spinor field is given by a column matrix, with $\bar{\psi}=\gamma^{0} \psi^{*}$ and the $\gamma^{\mu}$ are two by two Pauli matrices, anticommuting, self-adjoint and of square 1.

The Hilbert space of the quantum theory is the tensor product $\mathcal{H}=\mathcal{H}_{b} \otimes \mathcal{H}_{f}$ of the bosonic one $\mathcal{H}_{b}$ by the fermionic one $\mathcal{H}_{f}$. The quantum field $\phi(x)$ and its conjugate momentum $\pi(x)$ are operator valued distributions in $\mathcal{H}_{b}$ and the bosonic Hamiltonian is of the form

$$
H_{b}=\int_{S^{1}}:|\pi(x)|^{2}+\left|\partial_{1} \phi(x)\right|^{2}+m^{2}|\phi(x)|^{2}: d x
$$

where the Wick ordering takes care of an irrelevant additive constant. The fermionic Hilbert space $\mathcal{H}_{f}$ is given by the Dirac sea representation which simply corresponds to a suitable spin representation of the infinite dimensional Clifford algebra containing the fermionic quantum fields $\psi_{j}(x)$. The fermionic Hamiltonian is then the positive operator in $\mathcal{H}_{f}$ given by

$$
H_{f}=\int_{S^{1}}: \bar{\psi} \gamma^{1} i \partial \psi-m \bar{\psi} \psi:
$$


The full Hamiltonian of the non-interacting theory is acting in the Hilbert space $\mathcal{H}=\mathcal{H}_{b} \otimes \mathcal{H}_{f}$ and is the positive operator

$$
H=H_{b} \otimes 1+1 \otimes H_{f}
$$

This is were supersymmetry enters the scene in finding a self-adjoint square root of $H$ in the same way as the Dirac operator is a square root of the Laplacian in the case of finite dimensional manifolds. This square root, called the supercharge operator, is given by

$$
Q=\frac{1}{\sqrt{2}} \int_{S^{1}}\left(\psi_{1}(x)\left(\pi(x)-\partial \phi^{*}(x)-i m \phi(x)\right)+\psi_{2}(x)\left(\pi^{*}(x)-\partial \phi(x)-i m \phi^{*}(x)\right)+\text { h.c. }\right) d x
$$

where the symbol +h.c. means that one adds the hermitian conjugate.

The basic relation with spectral triples is then given by the following result ([54] Section IV).

Theorem 16.1. For any local region $\mathcal{O} \subset C$ let $\mathcal{A}(\mathcal{O})$ be the algebra of functions of quantum fields with support in $\mathcal{O}$ acting in the Hilbert space $\mathcal{H}$. Then the triple

$$
(\mathcal{A}(\mathcal{O}), \mathcal{H}, Q)
$$

is an even $\theta$-summable spectral triple, with $\mathbb{Z}_{2}$-grading given by the operator $\gamma=(-1)^{N_{f}}$ counting the parity of the fermion number operator $N_{f}$.

To be more specific the algebra $\mathcal{A}(\mathcal{O})$ is generated by the imaginary exponentials $e^{i\left(\phi(f)+\phi(f)^{*}\right)}$ and $e^{i\left(\pi(f)+\pi(f)^{*}\right)}$ for $f \in C_{c}^{\infty}(\mathcal{O})$. As shown in [54] Section IV.9. $\beta$, and exactly as in the case of discrete groups with exponential growth, one needs the entire cyclic cohomology rather than its finite dimensional version in order to obtain the Chern character of $\theta$-summable spectral triples. Indeed the index map is non polynomial in the above example of the Wess-Zumino model in two dimensions and the $K$-theory of the above local algebras is highly non-trivial. In fact it is in that framework that the JLO-cocycle was discovered by Jaffe-Lesniewski and Osterwalder [120].

It is an open problem to extend the above result to interacting theories in higher dimension and give a full computation of the $K$-theory of the local algebras as well as of the Chern character in entire cyclic cohomology. The results of Jaffe and his collaborators on constructive quantum field theory yield many interacting non-trivial examples of supersymmetric two dimensional models. Moreover the recent breakthrough of Puschnigg in the case of lattices of semi-simple Lie groups of rank one opens the way to the computation of the Chern character in entire cyclic cohomology.

\section{SpaCETIME AND THE STANDARD MODEL OF ELEMENTARY PARTICLES}

The standard model of elementary particle physics provides a surprising example of a spectral triple in the noncommutative setting, which in addition to the conditions of Definition 13.6 also has a real structure satisfying all the additional conditions of Definition 13.10.

The noncommutative geometry of the standard model developed in [61] (cf. also [44, 45], 73], [125]) gives a concise conceptual way to describe, through a simple mathematical structure, the full complexity of the input from physics. As we recall here, the model also allows for predictions. 


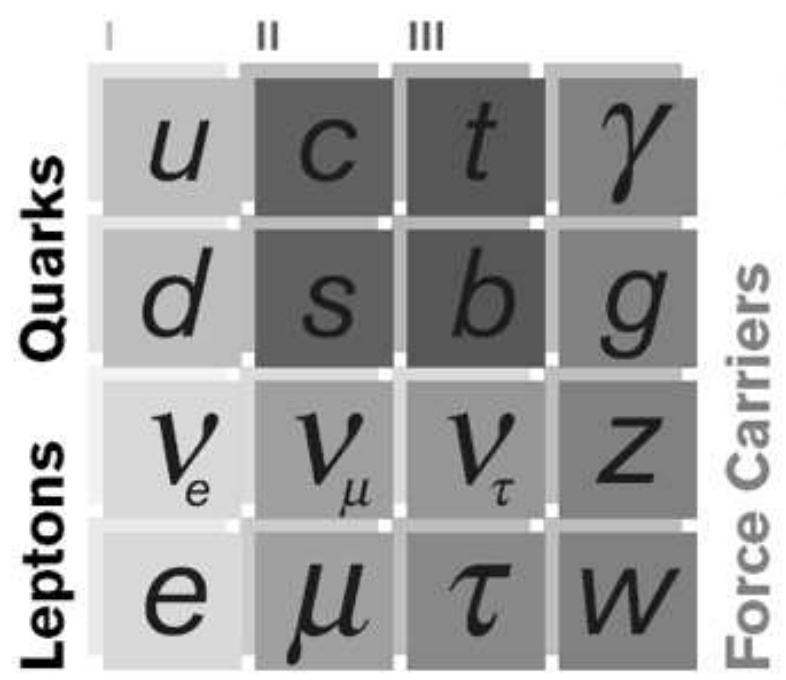

Three Generations of Matter

FiguRE 14. Elementary particles

The physics of the standard model can be described by a Lagrangian. We consider here the standard model minimally coupled to gravity, so that the Lagrangian we shall be concerned with is the sum

$$
\mathcal{L}=\mathcal{L}_{E H}+\mathcal{L}_{S M}
$$

of the Einstein-Hilbert Lagrangian $\mathcal{L}_{E H}$ and the standard model Lagrangian $\mathcal{L}_{S M}$.

The standard model Lagrangian $\mathcal{L}_{S M}$ has a very complicated expression, which, if written in full, might take a full page ( $c f$. e.g. [196]). It comprises five types of terms,

$$
\mathcal{L}_{S M}=\mathcal{L}_{G}+\mathcal{L}_{G H}+\mathcal{L}_{H}+\mathcal{L}_{G f}+\mathcal{L}_{H f},
$$

where the various terms involve:

- spin 1 bosons $G$ : the eight gluons, $\gamma, W^{ \pm}, Z$;

- spin 0 bosons $H$ such as the Higgs fields;

- spin $1 / 2$ fermions $f$ : quarks and leptons.

The term $\mathcal{L}_{G}$ is the pure gauge boson part, $\mathcal{L}_{G H}$ for the minimal coupling with the Higgs fields, and $\mathcal{L}_{H}$ gives the quartic Higgs self interaction. In addition to the coupling constants for the gauge fields, the fermion kinetic term $\mathcal{L}_{G f}$ contains the hypercharges $Y_{L}, Y_{R}$. These numbers, which are constant over generations, are assigned phenomenologically, so as to obtain the correct values of the electromagnetic charges. The term $\mathcal{L}_{H f}$ contains the Yukawa coupling of the Higgs fields with fermions. A more detailed and explicit description of the various terms of (17.2) is given in [54 $\S$ VI.5. $\beta$. See also [196].

The symmetry group of the Einstein-Hilbert Lagrangian $\mathcal{L}_{E H}$ by itself would be, by the equivalence principle, the diffeomorphism group $\operatorname{Diff}(X)$ of the space-time manifold. In the standard model Lagrangian $\mathcal{L}_{S M}$, on the other hand, the gauge theory has another huge symmetry group which is the group of local gauge transformations. According to our current 
understanding of elementary particle physics, this is given by

$$
G_{S M}(X)=\mathrm{C}^{\infty}(X, U(1) \times S U(2) \times S U(3)) .
$$

(At least in the case of a trivial principal bundle, e.g. when the spacetime manifold $X$ is contractible.)

Thus, when one considers the Lagrangian $\mathcal{L}$ of (17.1), the full symmetry group $G$ will be a semidirect product

$$
G(X)=G_{S M}(X) \rtimes \operatorname{Diff}(X) .
$$

In fact, a diffeomorphism of the manifold relabels the gauge parameters.

To achieve a geometrization of the standard model, one would like to be able to exhibit a space $X$ for which

$$
G(X)=\operatorname{Diff}(X)
$$

If such a space existed, then we would be able to say that the whole theory is pure gravity on $X$. However, it is impossible to find such a space $X$ among ordinary manifolds. In fact, a result of W. Thurston, D. Epstein and J. Mather ( $c f$. 156]) shows that the connected component of the identity in the diffeomorphism group of a (connected) manifold is a simple group (see [156] for the precise statement). A simple group cannot have a nontrivial normal subgroup, so it cannot have the structure of semi-direct product like $G(X)$ in (17.4).

However, it is possible to obtain a space with the desired properties among noncommutative spaces. What plays the role of the connected component of the identity in the diffeomorphism group $\operatorname{Diff}(X)$ in the noncommutative setting is the group $\operatorname{Aut}^{+}(\mathcal{A})$ of automorphisms of the (noncommutative) algebra that preserve the fundamental class in $K$-homology i.e. that can be implemented by a unitary compatible with the grading and real structure.

When the algebra $\mathcal{A}$ is not commutative, among its automorphisms there are, in particular, inner ones. They associate to an element $x$ of the algebra the element $u x u^{-1}$, for some $u \in \mathcal{A}$. Of course $u x u^{-1}$ is not, in general, equal to $x$ because the algebra is not commutative. The inner automorphisms form a normal subgroup of the group of automorphisms. Thus, we see that the group $\operatorname{Aut}^{+}(\mathcal{A})$ has in general the same type of structure as our desired group of symmetries $G(X)$, namely, it has a normal subgroup of inner automorphisms and it has a quotient. It is amusing how the physical and the mathematical vocabularies agree here: in physics one talks about internal symmetries and in mathematics one talks about inner automorphisms (one might as well call them internal automorphisms).

There is a very simple non commutative algebra $\mathcal{A}$ whose group of inner automorphisms corresponds to the group of gauge transformations $G_{S M}(X)$, and such that the quotient $\operatorname{Aut}^{+}(\mathcal{A}) / \operatorname{Inn}(\mathcal{A})$ corresponds exactly to diffeomorphisms ( $c f$. [181]). The noncommutative space is a product $X \times F$ of an ordinary spacetime manifold $X$ by a "finite noncommutative space" $F$. The noncommutative algebra $\mathcal{A}_{F}$ is a direct sum of the algebras $\mathbb{C}, \mathbb{H}$ (here denoting the quaternions), and $M_{3}(\mathbb{C})$ (the algebra of $3 \times 3$ complex matrices).

The algebra $\mathcal{A}_{F}$ corresponds to a finite space where the standard model fermions and the Yukawa parameters (masses of fermions and mixing matrix of Kobayashi Maskawa) determine the spectral geometry in the following manner. The Hilbert space $\mathcal{H}_{F}$ is finite-dimensional and admits the set of elementary fermions as a basis. This comprises the generations of 
quarks (down-up, strange-charmed, bottom-top),

$$
\begin{aligned}
& \begin{array}{llllllll}
u_{L} & u_{R} & d_{L} & d_{R} & \bar{u}_{L} & \bar{u}_{R} & \bar{d}_{L} & \bar{d}_{R}
\end{array} \\
& \begin{array}{llllllll}
c_{L} & c_{R} & s_{L} & s_{R} & \bar{c}_{L} & \bar{c}_{R} & \bar{s}_{L} & \bar{s}_{R}
\end{array} \\
& \begin{array}{llllllll}
t_{L} & t_{R} & b_{L} & b_{R} & \bar{t}_{L} & \bar{t}_{R} & \bar{b}_{L} & \bar{b}_{R},
\end{array}
\end{aligned}
$$

with the additional color index $(y, r, b)$, and the generations of leptons (electron, muon, tau, and corresponding neutrinos)

$$
\begin{array}{cccccc}
e_{L} & e_{R} & \nu_{L}^{e} & \bar{e}_{L} & \bar{e}_{R} & \bar{\nu}_{L}^{e} \\
\mu_{L} & \mu_{R} & \nu_{L}^{\mu} & \bar{\mu}_{L} & \bar{\mu}_{R} & \bar{\nu}_{L}^{\mu} \\
\tau_{L} & \tau_{R} & \nu_{L}^{\tau} & \bar{\tau}_{L} & \bar{\tau}_{R} & \bar{\nu}_{L}^{\tau}
\end{array}
$$

(We discuss here only the minimal standard model with no right handed neutrinos.)

The $\mathbb{Z} / 2$ grading $\gamma_{F}$ on the Hilbert space $\mathcal{H}_{F}$ has sign +1 on left handed particles (e.g. the $u_{L}, d_{L}$, etc.) and sign -1 on the right handed particles. The involution $J_{F}$ giving the real structure is the charge conjugation, namely, if we write $\mathcal{H}_{F}=\mathcal{E} \oplus \overline{\mathcal{E}}$, then $J_{F}$ acts on the fermion basis as $J_{F}(f, \bar{h})=(h, \bar{f})$. This satisfies $J_{F}^{2}=1$ and $J_{F} \gamma_{F}=\gamma_{F} J_{F}$, as should be for dimension $n=0$.

The algebra $\mathcal{A}_{F}$ admits a natural representation in $\mathcal{H}_{F}$ (see 62]). An element $(z, q, m) \in$ $\mathbb{C} \oplus \mathbb{H} \oplus M_{3}(\mathbb{C})$ acts as

$$
\begin{gathered}
(z, q, m) \cdot\left(\begin{array}{c}
u_{R} \\
d_{R}
\end{array}\right)=\left(\begin{array}{c}
z u_{R} \\
\bar{z} d_{R}
\end{array}\right) \quad(z, q, m) \cdot e_{R}=\bar{z} e_{R} \\
(z, q, m) \cdot\left(\begin{array}{c}
u_{L} \\
d_{L}
\end{array}\right)=q\left(\begin{array}{c}
u_{L} \\
d_{L}
\end{array}\right) \quad(z, q, m) \cdot\left(\begin{array}{c}
\nu_{L}^{e} \\
e_{L}
\end{array}\right)=q\left(\begin{array}{c}
\nu_{L}^{e} \\
e_{L}
\end{array}\right), \\
(z, q, m) \cdot\left(\begin{array}{c}
\bar{e}_{L} \\
\bar{e}_{R}
\end{array}\right)=\left(\begin{array}{c}
z \bar{e}_{L} \\
z \bar{e}_{R}
\end{array}\right) \\
(z, q, m) \cdot \bar{u}_{R}=m \bar{u}_{R} \quad(z, q, m) \cdot \bar{d}_{R}=m \bar{d}_{R}
\end{gathered}
$$

and similarly for the other generations. Here $q \in \mathbb{H}$ acts as multiplication by the matrix

$$
q=\left(\begin{array}{cc}
\alpha & \beta \\
-\bar{\beta} & \bar{\alpha}
\end{array}\right)
$$

where $q=\alpha+\beta j$, with $\alpha, \beta \in \mathbb{C}$. The matrix $m \in M_{3}(\mathbb{C})$ acts on the color indices $(y, r, b)$. The data $\left(\mathcal{A}_{F}, \mathcal{H}_{F}\right)$ can be completed to a spectral triple $\left(\mathcal{A}_{F}, \mathcal{H}_{F}, D_{F}\right)$ where the Dirac operator (in this finite dimensional case a matrix) is given by

$$
D_{F}=\left(\begin{array}{cc}
Y & 0 \\
0 & \bar{Y}
\end{array}\right)
$$

on $\mathcal{H}_{F}=\mathcal{E} \oplus \overline{\mathcal{E}}$, where $Y$ is the Yukawa coupling matrix, which combines the masses of the elementary fermions together with the Cabibbo-Kobayashi-Maskawa (CKM) quark mixing matrix. 
The fermionic fields acquire mass through the spontaneous symmetry breaking produced by the Higgs fields. The Yukawa coupling matrix takes the form $Y=Y_{q} \otimes 1 \oplus Y_{f}$, where the matrix $Y_{f}$ is of the form

$$
\left(\begin{array}{ccc}
0 & 0 & M_{e} \\
0 & 0 & 0 \\
M_{e}^{*} & 0 & 0
\end{array}\right),
$$

in the basis $\left(e_{R}, \nu_{L}, e_{L}\right)$ and successive generations, while $Y_{q}$ is of the form

$$
\left(\begin{array}{cccc}
0 & 0 & M_{u} & 0 \\
0 & 0 & 0 & M_{d} \\
M_{u}^{*} & 0 & 0 & 0 \\
0 & M_{d}^{*} & 0 & 0
\end{array}\right),
$$

in the basis given by $\left(u_{R}, d_{R}, u_{L}, d_{L}\right)$ and successive generations. In the case of the lepton masses, up to rotating the fields to mass eigenstates, one obtains a mass term for each fermion, and the off diagonal terms in $M_{e}$ can be reabsorbed in the definition of the fields. In the quark case, the situation is more complicated and the Yukawa coupling matrix can be reduced to the mass eigenvalues and the CKM quark mixing. By rotating the fields, it is possible to eliminate the off diagonal terms in $M_{u}$. Then $M_{d}$ satisfies $V M_{d} V^{*}=M_{u}$, where $V$ is the CKM quark mixing, given by a $3 \times 3$ unitary matrix

$$
V=\left(\begin{array}{ccc}
V_{u d} & V_{u s} & V_{u b} \\
V_{c d} & V_{c s} & V_{c b} \\
V_{t d} & V_{t s} & V_{t b}
\end{array}\right)
$$

acting on the charge $-e / 3$ quarks (down, strange, bottom). The entries of this matrix can be expressed in terms of three angles $\theta_{12}, \theta_{23}, \theta_{13}$ and a phase, and can be determined experimentally from weak decays and deep inelastic neutrino scatterings.

The detailed structure of the Yukawa coupling matrix $Y$ (in particular the fact that color is not broken) allows one to check that the finite geometry $\left(\mathcal{A}_{F}, \mathcal{H}_{F}, D_{F}\right)$ satisfies all the axioms of Definition 13.10 for a noncommutative spectral manifold. The key point is that elements $a \in \mathcal{A}_{F}$ and $\left[D_{f}, a\right]$ commute with $J_{F} \mathcal{A}_{F} J_{F}$. These operators preserve the subspace $\mathcal{E} \subset \mathcal{H}_{F}$. On this subspace, for $b=(z, q, m)$, the action of $J_{F} b^{*} J_{F}$ is by multiplication by $z$ or by the transpose $m^{t}$. It is then not hard to check explicitly the commutation with $a$ or $[D, a](c f$. $54 \S$ VI.5. $\delta$ ). By exchanging the roles of $a$ and $b$, one sees analogously that $a$ commutes with $J_{F} b J_{F}$ and $\left[D, J_{F} b J_{F}\right]$ on $\overline{\mathcal{E}}$, hence the desired commutation relations hold on all of $\mathcal{H}_{F}$.

We can then consider the product $X \times F$, where $X$ is an ordinary 4-dimensional Riemannian spin manifold and $F$ is the finite geometry described above. This product geometry is a spectral triple $(\mathcal{A}, \mathcal{H}, D)$ obtained as the cup product of a triple $\left(\mathcal{C}^{\infty}(X), L^{2}(X, S), D_{1}\right)$, where $D_{1}$ is the Dirac operator on $X$ acting on square integrable spinors in $L^{2}(X, S)$, with the spectral triple $\left(\mathcal{A}_{F}, \mathcal{H}_{F}, D_{F}\right)$ described above. Namely, the resulting (smooth) algebra and Hilbert space are of the form

$$
\mathcal{A}_{\infty}=\mathcal{C}^{\infty}\left(X, \mathcal{A}_{F}\right) \quad \mathcal{H}=L^{2}(X, S) \otimes \mathcal{H}_{F},
$$

and the Dirac operator is given by

$$
D=D_{1} \otimes 1+\gamma \otimes D_{F},
$$


where $\gamma$ is the usual $\mathbb{Z} / 2$ grading on the spinor bundle $S$. The induced $\mathbb{Z} / 2$ grading on $\mathcal{H}$ is the tensor product $\gamma \otimes \gamma_{F}$, and the real structure is given by $J=C \otimes J_{F}$, where $C$ is the charge conjugation operator on spinors.

Notice that, so far, we have only used the information on the fermions of the standard model. We'll see now that the bosons, with the correct quantum numbers, are deduced as inner fluctuations of the metric of the spectral triple $(\mathcal{A}, \mathcal{H}, D)$.

It is a general fact that, for noncommutative geometries $(\mathcal{A}, \mathcal{H}, D)$, one can consider inner fluctuations of the metric of the form

$$
D \mapsto D+A+J A J^{-1}
$$

where $A$ is of the form

$$
A=\sum a_{i}\left[D, a_{i}^{\prime}\right] \quad a_{i}, a_{i}^{\prime} \in \mathcal{A}
$$

In the case of the standard model, a direct computation of the inner fluctuations gives the standard model gauge bosons $\gamma, W^{ \pm}, Z$, the eight gluons and the Higgs fields $\varphi$ with accurate quantum numbers ( $c f$. 61]). In fact, a field $A$ of the form (17.11) can be separated in a "discrete part" $A^{(0,1)}=\sum a_{i}\left[\gamma \otimes D_{F}, a_{i}^{\prime}\right]$ and a continuous part $A^{(1,0)}=\sum a_{i}\left[D_{1} \otimes 1, a_{i}^{\prime}\right]$, with $a_{i}=\left(z_{i}, q_{i}, m_{i}\right)$ and $a_{i}^{\prime}=\left(z_{i}^{\prime}, q_{i}^{\prime}, m_{i}^{\prime}\right), q_{i}=\alpha_{i}+\beta_{i} j$ and $q_{i}^{\prime}=\alpha_{i}^{\prime}+\beta_{i}^{\prime} j$. The discrete part gives a quaternion valued function

$$
q(x)=\sum z_{i}\left(\left(\alpha_{i}^{\prime}-z_{i}^{\prime}\right)+z_{i} \beta_{i}^{\prime} j\right)=\varphi_{1}+\varphi_{2} j
$$

which provides the Higgs doublet. The continuous part gives three types of fields:

- A $U(1)$ gauge field $U=\sum z_{i} d z_{i}^{\prime}$

- An $S U(2)$ gauge field $Q=\sum q_{i} d q_{i}^{\prime}$

- A $U(3)$ gauge field $M=\sum m_{i} d m_{i}^{\prime}$, which can be reduced to an $S U(3)$ gauge field $M^{\prime}$ by subtracting the scalar part of the overall gauge field which eliminates inessential fluctuations that do not change the metric.

The resulting internal fluctuation of the metric $A+J A J^{-1}$ is then of the form (cf. 61]

$$
\left(\begin{array}{ccc}
-2 U & 0 & 0 \\
0 & Q_{11}-U & Q_{12} \\
0 & Q_{21} & Q_{22}-U
\end{array}\right)
$$

on the basis of leptons $\left(e_{R}, \nu_{L}, e_{L}\right)$ and successive generations, and

$$
\left(\begin{array}{cccc}
\frac{4}{3} U+M^{\prime} & 0 & 0 & 0 \\
0 & \frac{-2}{3} U+M^{\prime} & 0 & 0 \\
0 & 0 & Q_{11}+\frac{1}{3} U+M^{\prime} & Q_{12} \\
0 & 0 & Q_{21} & Q_{22}+\frac{1}{3} U+M^{\prime}
\end{array}\right)
$$

on the basis of quarks given by $\left(u_{R}, d_{R}, u_{L}, d_{L}\right)$ and successive generations. A striking feature that these internal fluctuations exhibit is the fact that the expressions above recover all the exact values of the hypercharges $Y_{L}, Y_{R}$ that appear in the fermion kinetic term of the standard model Lagrangian.

Finally, one can also recover the bosonic part of the standard model Lagragian from a very general principle, the spectral action principle of Chamseddine-Connes (cf. 44], 45], 46]). 
The result is that the Hilbert-Einstein action functional for the Riemannian metric, the YangMills action for the vector potentials, and the self interaction and the minimal coupling for the Higgs fields all appear with the correct signs in the asymptotic expansion for large $\Lambda$ of the number $N(\Lambda)$ of eigenvalues of $D$ which are $\leq \Lambda$ (cf. [4]),

$$
N(\Lambda)=\# \text { eigenvalues of } D \text { in }[-\Lambda, \Lambda] \text {. }
$$

The spectral action principle, applied to a spectral triple $(\mathcal{A}, \mathcal{H}, D)$, can be stated as saying that the physical action depends only on $\operatorname{Spec}(D) \subset \mathbb{R}$. This spectral datum corresponds to the data $(\mathcal{H}, D)$ of the spectral triple, independent of the action of $\mathcal{A}$. Different $\mathcal{A}$ that correspond to the same spectral data can be thought of as the noncommutative analog of isospectral Riemannian manifolds ( $c f$. the discussion of isospectral deformations in Section 18). A natural expression for an action that depends only on $\operatorname{Spec}(D)$ and is additive for direct sums of spaces is of the form

$$
\operatorname{Tr} \chi\left(\frac{D}{\Lambda}\right)+\langle\psi, D \psi\rangle
$$

where $\chi$ is a positive even function and $\Lambda$ is a scale.

In the case of the standard model, this formula (17.13) is applied to the full "metric" including the internal fluctuations and gives the full standard model action minimally coupled with gravity. The Fermionic part of the action (17.13) gives (cf. 44], 45])

$$
\langle\psi, D \psi\rangle=\int_{X}\left(\mathcal{L}_{G f}+\mathcal{L}_{H f}\right) \sqrt{|g|} d^{4} x .
$$

The bosonic part of the action (17.13) evaluated via heat kernel invariants gives the standard model Lagrangian minimally coupled with gravity. Namely, one writes the function $\chi\left(\frac{D}{\Lambda}\right)$ as the superposition of exponentials. One then computes the trace by a semiclassical approximation from local expressions involving the familiar heat equation expansion. This delivers all the correct terms in the action ( $c f$. 45] for an explict calculation of all the terms involved). Notice that here one treats the spacetime manifold $X$ in the Euclidean signature. The formalism of spectral triple can be extended in various ways to the Lorentzian signature ( $c f$. e.g. [115]). Perhaps the most convenient choice is to drop the self-adjointness condition for $D$ while still requiring $D^{2}$ to be self-adjoint.

\section{ISOSPECTRAL DEFORMATIONS}

A very rich class of examples of noncommutative manifolds is obtained by considering isospectral deformations of a classical Riemannian manifold. These examples satisfy all the axioms of ordinary Riemannian geometry ( $c f$. 61] ) except commutativity. They are obtained by the following result (Connes-Landi [72]):

Theorem 18.1. Let $M$ be a compact Riemannian spin manifold. Then if the isometry group of $M$ has rank $r \geq 2, M$ admits a non-trivial one parameter isospectral deformation to noncommutative geometries $M_{\theta}$.

The main idea of the construction is to deform the standard spectral triple describing the Riemannian geometry along a two torus embedded in the isometry group, to a family of spectral triples describing non-commutative geometries. 
More precisely, under the assumption on the rank of the group of isometries of the compact spin manifold $X$, there exists a two-torus

$$
T^{2} \subset \operatorname{Isom}(X)
$$

where we identify $T^{2}=\mathbb{R}^{2} /(2 \pi \mathbb{Z})^{2}$. Let $U(s)$ be the unitary operators in this subgroup of isometries, for $s=\left(s_{1}, s_{2}\right) \in T^{2}$, acting on the Hilbert space $\mathcal{H}=L^{2}(X, S)$ of the spectral triple

$$
\left(C^{\infty}(X), L^{2}(X, S), D, J\right)
$$

Equivalently, we write $U(s)=\exp \left(i\left(s_{1} P_{1}+s_{2} P_{2}\right)\right)$, where $P_{i}$ are the corresponding Lie algebra generators, with $\operatorname{Spec}\left(P_{i}\right) \subset \mathbb{Z}$, satisfying $\left[D, P_{i}\right]=0$ and $P_{i} J=-J P_{i}$, so that $[U(s), D]=[U(s), J]=0$.

The action $\alpha_{s}(T)=U(s) T U(s)^{-1}$ has the following property. Any operator $T$ such that the map $s \mapsto \alpha_{s}(T)$ is smooth can be uniquely written as a norm convergent series

$$
T=\sum_{n_{1}, n_{2} \in \mathbb{Z}} \hat{T}_{n_{1}, n_{2}}
$$

where each term $\hat{T}_{n_{1}, n_{2}}$ is an operator of bi-degree $\left(n_{1}, n_{2}\right)$, that is,

$$
\alpha_{s}\left(\hat{T}_{n_{1}, n_{2}}\right)=\exp \left(i\left(s_{1} n_{1}+s_{2} n_{2}\right)\right) \hat{T}_{n_{1}, n_{2}}
$$

for each $s=\left(s_{1}, s_{2}\right) \in T^{2}$, and the sequence of norms $\left\|\hat{T}_{n_{1}, n_{2}}\right\|$ is of rapid decay.

This property makes it possible to define left and right twists for such operators $T$, defined as

$$
\ell(T):=\sum_{n_{1}, n_{2}} \hat{T}_{n_{1}, n_{2}} \exp \left(2 \pi i \theta n_{2} P_{1}\right)
$$

and

$$
r(T):=\sum_{n_{1}, n_{2}} \hat{T}_{n_{1}, n_{2}} \exp \left(2 \pi i \theta n_{1} P_{2}\right)
$$

Both series still converge in norm, since the $P_{i}$ are self-adjoint operators.

It is then possible to introduce a (left) deformed product

$$
x * y=\exp \left(2 \pi i \theta n_{1}^{\prime} n_{2}\right) x y
$$

for $x$ a homogeneous operator of bi-degree $\left(n_{1}, n_{2}\right)$ and $y$ a homogeneous operator of bi-degree $\left(n_{1}^{\prime}, n_{2}^{\prime}\right)$. A (right) deformed product is similarly defined by setting $x *_{r} y=\exp \left(2 \pi i \theta n_{1} n_{2}^{\prime}\right) x y$. These deformed products satisfy $\ell(x) \ell(y)=x * y$ and $r(x) r(y)=x *_{r} y$.

The deformed spectral triples are then obtained by maintaining the same Hilbert space $\mathcal{H}=$ $L^{2}(X, S)$ and Dirac operator $D$, while modifying the algebra $C^{\infty}(X)$ to the non-commutative algebra $\mathcal{A}_{\theta}:=\ell\left(C^{\infty}(X)\right)$ and the involution $J$ that defines the real structure to $J_{\theta}:=$ $\exp \left(2 \pi i \theta P_{1} P_{2}\right) J$. 


\section{Algebraic Deformations}

There is a very general context in which one constructs noncommutative spaces via deformations of commutative algebras. Unlike the isospectral deformations discussed in Section 18. here one proceeds mostly at a formal algebraic level, without involving the operator algebra structure and without invoking the presence of a Riemannian structure.

The idea of deformation quantization originates in the idea that classical mechanics has as setting a smooth manifold (phase space) with a symplectic structure, which defines a Poisson bracket $\{$,$\} . The system is quantized by deforming the pointwise product in the algebra$ $\mathcal{A}=\mathcal{C}^{\infty}(M)$ (or in a suitable subalgebra) to a family $* \hbar$ of products satisfying $f * \hbar g \rightarrow f g$ as $\hbar \rightarrow 0$, which are associative but no longer necessarily commutative. These are also required to satisfy

$$
\frac{f * \hbar g-g * \hbar f}{i \hbar} \rightarrow\{f, g\},
$$

as $\hbar \rightarrow 0$, namely, the ordinary product is deformed in the direction of the Poisson bracket. On the algebra $\mathcal{C}^{\infty}(M)$ a Poisson bracket is specified by assigning a section $\Lambda$ of $\Lambda^{2}(T M)$ with the property that

$$
\{f, g\}=\langle\Lambda, d f \wedge d g\rangle
$$

satisfies the Jacobi identity. Typically, this produces a formal deformation: a formal power series in $\hbar$. Namely, the deformed product can be written in terms of a sequence of bidifferential operators $B_{k}$ satisfying

$$
f * g=f g+\hbar B_{1}(f, g)+\hbar^{2} B_{2}(f, g)+\cdots
$$

Under this perspective, there is a good understanding of formal deformations. For instance, Kontsevich [132] proved that formal deformations always exist, by providing an explicit combinatorial formula that generates all the $\left\{B_{2}, B_{3}, \ldots\right\}$ in the expansion from the $B_{1}$, hence in terms of the Poisson structure $\Lambda$. The formal solution (19.1) can then be written as

$$
\sum_{n=0}^{\infty} \hbar^{n} \sum_{\Gamma \in G[n]} \omega_{\Gamma} B_{\Gamma, \Lambda}(f, g),
$$

where $G[n]$ is a set of $(n(n+1))^{n}$ labeled graphs with $n+2$ vertices and $n$ edges, $\omega_{\Gamma}$ is a coeffcient obtained by integrating a differential form (depending on the graph $\Gamma$ ) on the configuration space of $n$ distinct points in the upper half plane, and $B_{\Gamma, \Lambda}$ is a bi-differential operator whose coefficients are derivatives of $\Lambda$ of orders specified by the combinatorial information of the graph $\Gamma$.

A setting of deformation quantization which is compatible with $C^{*}$-algebras was developed by Rieffel in [176]. We recall briefly Rieffel's setting. For simplicity, we restrict to the simpler case of a compact manifold.

Definition 19.1. A strict (Rieffel) deformation quantization of $\mathcal{A}=\mathcal{C}^{\infty}(M)$ is obtained by assigning an associative product $*_{\hbar}$, an involution (depending on $\hbar$ ) and a $C^{*}{ }^{-n o r m}\|\cdot\|_{\hbar}$ on $\mathcal{A}$, for $\hbar \in I$ (some interval containing zero), such that:

(i) For $\hbar=0$ these give the $C^{*}$-algebra $C(M)$, 
(ii) For all $f, g \in \mathcal{A}$, as $\hbar \rightarrow 0$,

$$
\left\|\frac{f * \hbar g-g * \hbar f}{i \hbar}-\{f, g\}\right\|_{\hbar} \rightarrow 0 .
$$

One denotes by $\mathcal{A}_{\hbar}$ the $C^{*}$-algebra obtained by completing $\mathcal{A}$ in the norm $\|\cdot\|_{\hbar}$.

The functions of $\hbar$ are all supposed to be analytic, so that formal power series expansions make sense.

Remark 19.2. The notion of a strict deformation quantization should be regarded as a notion of integrability for a formal solutions.

Rieffel also provides a setting for compatible actions by a Lie group of symmetries, and proves that non-commutative tori (also of higher rank) are strict deformation quantizations of ordinary tori, that are compatible with the action of the ordinary torus as group of symmetry. Typically, for a given Poisson structure, strict deformation quantizations are not unique. This happens already in the case of tori.

In the same paper [176, Rieffel uses a basic result of Wassermann 199 to produce an example where formal solutions are not integrable. The example is provided by the two-sphere $S^{2}$. There is on $S^{2}$ a symplectic structure, and a corresponding Poisson structure $\Lambda$ which is invariant under $S O(3)$. Rieffel proves the following striking result (Theorem 7.1 of [176]):

Theorem 19.3. There are no $S O(3)$-invariant strict deformations of the ordinary product on $\mathcal{C}^{\infty}\left(S^{2}\right)$ in the direction of the $S O(3)$-invariant Poisson structure.

In fact, the proof of this result shows more, namely that no $S O(3)$-invariant deformation of the ordinary product in $C\left(S^{2}\right)$ can produce a non-commutative $C^{*}$-algebra. This rigidity result reflects a strong rigidity result for $S U(2)$ proved by Wassermann [199, namely the only ergodic actions of $S U(2)$ are on von Neumann algebras of type I. The interest of this result lies in the fact that there are formal deformations of the Poisson structure that are $S O(3)$-invariant (see e.g. [11], [17]), but these only exist as a formal power series in the sense of (19.1) and, by the results of Wassermann and Rieffel are not integrable.

Summarizing, we have the following type of phenomenon: on the one hand we have formal solutions, formal deformation quantizations about which a lot is known, but for which, in general, there may not be an integrability result. More precisely, when we try to pass from formal to actual solutions, there are cases where existence fails (the sphere), and others (tori) where uniqueness fails. The picture that emerges is remarkably similar to the case of formal and actual solutions of ordinary differential equations.

It is very instructive to build an analogy between the problem of ambiguity for formal solutions of ODE's and the present situation of formal non-commutative spaces and actual non-commutative spaces. The main conclusion to be drawn from this analogy is that there ought to be a theory of ambiguity which formulates precisely the relation between the formal non-commutative geometry and its integrated $\left(C^{*}\right.$-algebraic) version.

To illustrate this concept, we take a closer look at the analogous story in the theory of ODE's. A good reference for a modern viewpoint is [172. A formal solution of a differential equation is a power series expansion: for instance $\sum_{n=0}^{\infty}(-1)^{n} n ! x^{n+1}$ is a formal solution of the Euler equation $x^{2} y^{\prime}+y=x$. Convergent series give rise to actual solutions, and more 
involved summation processes such as Borel summation can be used to transform a given formal solution of an analytic ODE into an actual solution on a sufficiently narrow sector in $\mathbb{C}$ of sufficiently small radius, but such solution is in general not unique. It is known from several classical methods that some divergent series can be "summed" modulo a function with exponential decrease of a certain order. This property (Gevrey summability) is also satisfied by formal solutions of analytic ODE's, and, stated in a more geometric fashion, it is essentially a cohomological condition. It also shows that, whereas on small sectors one has existence of actual solutions but not uniqueness, on large sectors one gains uniqueness, at the cost of possibly loosing existence. A complete answer to summability of formal solutions can then be given in terms of a more refined multi-summability (combining Gevrey series and functions of different order) and the Newton polygon of the equation.

The general flavor of this theory is surprisingly similar to the problem of formal solutions in non-commutative geometry. It is to be expected that an ambiguity theorem exists, which accounts for the cases of lack of uniqueness, or lack of existence, of actual solutions illustrated by the results of Rieffel.

Already in dealing with our first truly non-trivial example of noncommutative spaces, the noncommutative tori, we encountered subtleties related to the difference between the quotient and the deformation approach to the construction of non-commutative spaces.

In fact, the non-commutative tori we described in Section [6] admit a description as algebras obtained as deformations of the ordinary product of functions, by setting

$$
(f * g)(x, y):=\left(e^{2 \pi i \theta \frac{\partial}{\partial x} \frac{\partial}{\partial y^{\prime}}} f(x, y) g\left(x^{\prime}, y^{\prime}\right)\right)_{x=x^{\prime}, y=y^{\prime}}=\sum \frac{(i 2 \pi \theta)^{n}}{n !} D_{1}^{n} f D_{2}^{n} g .
$$

Notice however that while $U \frac{\partial}{\partial U}$ and $V \frac{\partial}{\partial V}$ are derivations for the algebra of the noncommutative torus, this is not the case for $\frac{\partial}{\partial U}$ and $\frac{\partial}{\partial V}$. The same holds for the quantum plane ( $c f$. [146]) whose algebra of coordinates admits two generators $u, v$ with relation

$$
u v=q v u \text {. }
$$

These generators can be rotated $(u \mapsto \lambda u, v \mapsto \mu v)$ without affecting the presentation but translations of the generators are not automorphisms of the algebra. In other words, one can view the non-commutative torus as a deformation of an ordinary torus, which in turn is a quotient of the classical plane $\mathbb{R}^{2}$ by a lattice of translations, but the action of translations does not extend to the quantum plane. This is an instance of the fact that the general operations of quotient and deformation, in constructing non-commutative spaces, do not satisfy any simple compatibility rules and need to be manipulated with care.

Moreover, phenomena like the Morita equivalence between, for instance $\theta$ and $1 / \theta$, are not detectable in a purely deformation theoretic perturbative expansion like the one given by the Moyal product (19.2). They are non-perturbative and cannot be seen at the perturbative level of the star product.

In this respect, a very interesting recent result is that of Gayral, Gracia-Bondia, Iochum, Schücker, and Varilly, 106, where they consider a version of the structure of spectral triple for non-compact spaces. In that case, for instance, one no longer can expect the Dirac operator to have compact resolvent and one can only expect a local version to hold, e.g. $a(D-i)^{-1}$ is compact for $a \in \mathcal{A}$. Other properties of Definitions [13.6 and 13.10] are easily 
adapted to a "local version" but become more difficult to check than in the compact case. They show that the Moyal product deformation of $\mathbb{R}^{2 n}$ fits in the framework of spectral triples and provides an example of such non-unital spectral triples. Thus, it appears that the structure of noncommutative Riemannian geometry provided by spectral triples should adapt nicely to some classes of algebraic deformations.

It appears at first that spectral triples may not be the right type of structure to deal with noncommutative spaces associated to algebraic deformations, because it corresponds to a form of Riemannian geometry, while many such spaces originate from Kähler geometry. However, the Kähler structure can often be also encoded in the setting of spectral triple, for example by considering also a second Dirac operator, as in [26] or through the presence of a Lefschetz operator as in 91 .

Noncommutative spaces obtained as deformations of commutative algebras fit in the context of a well developed algebraic theory of noncommutative spaces (cf. e.g. 133] 134] 143] [144] [146] 178 [179, 189]). This theory touches on a variety of subjects like quantum groups and the deformation approach to non-commutative spaces and is interestingly connected to the theory of mirror symmetry. However, it is often not clear how to integrate this approach with the functional analytic theory of non-commutative geometry briefly summarized in section 2 , Only recently, several results confirmed the existence of a rich interplay between the algebraic and functional analytic aspects of noncommutative geometry, especially through the work of Connes and Dubois-Violette ( $c f$. 69, [70, [71]) and of Polishchuk (cf. [168]). Also, the work of Chakraborty and Pal 42] and Connes [65] and more recently of van Suijlekom, Dabrowski, Landi, Sitarz, and Varilly [191, [192] showed that quantum groups fit very nicely within the framework of noncommutative geometry described by spectral triples, contrary to what was previously belived. Ultimately, successfully importing tools from the theory of operator algebras into the realm of algebraic geometry might well land within the framework of what Manin refers to as a "second quantization of algebraic geometry".

\section{Quantum Groups}

For a long time it was widely believed that quantum groups could not fit into the setting of noncommutative manifolds defined in terms of spectral geometry. On the contrary, recent work of Chakraborti and Pal showed in 42] that the quantum group $S U_{q}(2)$, for $0 \leq q<1$, admits a spectral triple with Dirac operator that is equivariant with respect to its own (co)action.

The algebra $\mathcal{A}$ of functions on the quantum group $S U_{q}(2)$ is generated by two elements $\alpha$ and $\beta$ with the relations

$$
\begin{gathered}
\alpha^{*} \alpha+\beta^{*} \beta=1, \quad \alpha \alpha^{*}+q^{2} \beta \beta^{*}=1, \\
\alpha \beta=q \beta \alpha, \quad \alpha \beta^{*}=q \beta^{*} \alpha, \quad \beta^{*} \beta=\beta \beta^{*} .
\end{gathered}
$$

By the representation theory of the quantum group $S U_{q}(2)$ ( $c f$. 130]) there exists a Hilbert space $\mathcal{H}$ with orthonormal basis $e_{i j}^{(n)}, n \in \frac{1}{2} \mathbb{N}, i, j \in\{-n, \ldots, n\}$, and a unitary representation 


$$
\begin{aligned}
& \alpha e_{i j}^{(n)}=a_{+}(n, i, j) e_{i-1 / 2, j-1 / 2}^{(n+1 / 2)}+a_{-}(n, i, j) e_{i-1 / 2, j-1 / 2}^{(n-1 / 2)} \\
& \beta e_{i j}^{(n)}=b_{+}(n, i, j) e_{i+1 / 2, j-1 / 2}^{(n+1 / 2)}+b_{-}(n, i, j) e_{i+1 / 2, j-1 / 2}^{(n-1 / 2)}
\end{aligned}
$$

with coefficients

$$
\begin{aligned}
& a_{+}(n, i, j)=q^{2 n+i+j+1} Q(2 n-2 j+2,2 n-2 i+2,4 n+2,4 n+4) \\
& a_{-}(n, i, j)=Q(2 n+2 j, 2 n+2 i, 4 n, 4 n+2) \\
& b_{+}(n, i, j)=-q^{n+j} Q(2 n-2 j+2,2 n+2 i+2,4 n+2,4 n+4) \\
& b_{-}(n, i, j)=q^{n+i} Q(2 n+2 j, 2 n-2 i, 4 n, 4 n+2),
\end{aligned}
$$

where we use the notation

$$
Q(n, m, k, r)=\frac{\left(1-q^{n}\right)^{1 / 2}\left(1-q^{m}\right)^{1 / 2}}{\left(1-q^{k}\right)^{1 / 2}\left(1-q^{r}\right)^{1 / 2}} .
$$

Consider then, as in 65], the operator

$$
D e_{i j}^{(n)}=\left\{\begin{array}{rr}
-2 n & n \neq i \\
2 n & n=i .
\end{array}\right.
$$

More generally, one can consider operators of the form $D e_{i j}^{(n)}=d(n, i) e_{i j}^{(n)}$, as in [42, with $d(n, i)$ satisfying the conditions $d(n+1 / 2, i+1 / 2)-d(n, i)=O(1)$ and $d(n+1 / 2, i-1 / 2)-$ $d(n, i)=O(n+i+1)$. Then one has the following result (Chakraborti-Pal [42]):

Theorem 20.1. The data $(\mathcal{A}, \mathcal{H}, D)$ ad above define an $S U_{q}(2)$ equivariant odd 3-summable spectral triple.

The equivariance condition means that there is an action on $\mathcal{H}$ of the enveloping algebra $\mathcal{U}=U_{q}(\mathrm{SL}(2))$, which commutes with the Dirac operator $D$. This is generated by operators

$$
\begin{aligned}
& k e_{i j}^{(n)}=q^{j} e_{i j}^{(n)} \\
& e e_{i j}^{(n)}=q^{-n+1 / 2}\left(1-q^{2(n+j+1)}\right)^{1 / 2}\left(1-q^{2(n-j)}\right)^{1 / 2}\left(1-q^{2}\right)^{-1} e_{i j+1}^{(n)},
\end{aligned}
$$

satisfying the relations

$$
k e=q e k, \quad k f=q^{-1} f k, \quad[e, f]=\frac{k^{2}-k^{-2}}{q-q^{-1}},
$$

with $f=e^{*}$, and with coproduct

$$
\Delta(k)=k \otimes k, \quad \Delta(e)=k^{-1} \otimes e+e \otimes k, \quad \Delta(f)=k^{-1} \otimes f+f \otimes k .
$$

It is interesting that, while the classical $S U(2)$ is of (topological and metric) dimension three, the topological dimension of the algebra $\mathcal{A}$ of $S U_{q}(2)$ drops to one ( $c f$. [42]), but the metric dimension of the spectral triple remains equal to three as in the classical case.

Chakraborti and Pal showed in 42 that the Chern character of the spectral triple is nontrivial. Moreover, Connes in 65] gave an explicit formula for its local index cocycle, where a delicate calculation provides the cochain whose coboundary is the difference between the 
Chern character and the local version in terms of remainders in the rational approximation to the logarithmic derivative of the Dedekind eta function.

The local index formula is obtained by constructing a symbol map

$$
\rho: \mathcal{B} \rightarrow C^{\infty}\left(S_{q}^{*}\right)
$$

where the algebra $C^{\infty}\left(S_{q}^{*}\right)$ gives a noncommutative version of the cosphere bundle, with a restriction map $r: C^{\infty}\left(S_{q}^{*}\right) \rightarrow C^{\infty}\left(D_{q+}^{2} \times D_{q-}^{2}\right)$ to the algebra of two noncommutative disks. Here $\mathcal{B}$ is the algebra generated by the elements $\delta^{k}(a), a \in \mathcal{A}$, with $\delta(a)=[|D|, a]$. On the cosphere bundle there is a geodesic flow, induced by the group of automorphisms $a \mapsto e^{i t|D|} a e^{-i t|D|}$. Then $\rho(b)^{0}$ denotes the component of degree zero with respect to the grading induced by this flow.

The algebra $C^{\infty}\left(D_{q}^{2}\right)$ is an extension

$$
0 \rightarrow \mathcal{S} \rightarrow C^{\infty}\left(D_{q}^{2}\right) \stackrel{\sigma}{\rightarrow} C^{\infty}\left(S^{1}\right) \rightarrow 0
$$

where the ideal $\mathcal{S}$ is the algebra of rapidly decaying matrices. There are linear functionals $\tau_{0}$ and $\tau_{1}$ on $C^{\infty}\left(D_{q}^{2}\right)$

$$
\begin{gathered}
\tau_{1}(a)=\frac{1}{2 \pi} \int_{0}^{2 \pi} \sigma(a) d \theta, \\
\tau_{0}(a)=\lim _{N \rightarrow \infty} \sum_{k=0}^{N}\left\langle a \epsilon_{k}, \epsilon_{k}\right\rangle-\tau_{1}(a) N,
\end{gathered}
$$

where $\tau_{0}$ is defined in terms of the representation of $C^{\infty}\left(D_{q}^{2}\right)$ on the Hilbert space $\ell^{2}(\mathbb{N})$ with o.n. basis $\left\{\epsilon_{k}\right\}$.

Recall that (cf. [54) a cycle $\left(\Omega, d, \int\right)$ is a triple with where $(\Omega, d)$ is a graded differential algebra, and $\int: \Omega^{n} \rightarrow \mathbb{C}$ is a closed graded trace on $\Omega$. A cycle over an algebra $\mathcal{A}$ is given by a cycle $\left(\Omega, d, \int\right)$ together with a homomorphism $\rho: \mathcal{A} \rightarrow \Omega^{0}$.

In the case of the algebra $\mathcal{A}$ of $S U_{q}(2)$, a cycle $\left(\Omega, d, \int\right)$ is obtained in 65] by considering $\Omega^{1}=\mathcal{A} \oplus \Omega^{(2)}\left(S^{1}\right)$, with $\Omega^{(2)}\left(S^{1}\right)$ the space of weight two differential forms $f(\theta) d \theta^{2}$, with the $\mathcal{A}$-bimodule structure

$$
a(\xi, f)=(a \xi, \sigma(a) f) \quad(\xi, f) a=\left(\xi a,-i \sigma(\xi) \sigma(a)^{\prime}+f \sigma(a)\right),
$$

with differential

$$
d a=\partial a+\frac{1}{2} \sigma(a)^{\prime \prime} d \theta^{2}
$$

with $\partial$ the derivation $\partial=\partial_{\beta}-\partial_{\alpha}$, and

$$
\int(\xi, f)=\tau(\xi)+\frac{1}{2 \pi i} \int f d \theta
$$

where $\tau(a)=\tau_{0}\left(r_{-}\left(a^{(0)}\right)\right)$, with $a^{(0)}$ the component of degree zero for $\partial$ and $r_{-}$the restriction to $C^{\infty}\left(D_{q-}^{2}\right)$. This definition of the cycle corrects for the fact that $\tau$ itself (as well as $\tau_{0}$ ) fails to be a trace.

The following result then holds (Connes 65]):

Theorem 20.2. (1) The spectral triple $(\mathcal{A}, \mathcal{H}, D)$ of Theorem 20.1 has dimension spectrum $\Sigma=\{1,2,3\}$. 
(2) The residue formula for pseudodifferential operators $a \in \mathcal{B}$ in terms of their symbol is given by

$$
\begin{aligned}
& f a|D|^{-3}=\left(\tau_{1} \otimes \tau_{1}\right)\left(r \rho(a)^{0}\right) \\
& f a|D|^{-2}=\left(\tau_{1} \otimes \tau_{0}+\tau_{0} \otimes \tau_{1}\right)\left(r \rho(a)^{0}\right) \\
& \text { f } a|D|^{-1}=\left(\tau_{0} \otimes \tau_{0}\right)\left(r \rho(a)^{0}\right)
\end{aligned}
$$

(3) The character $\chi\left(a_{0}, a_{1}\right)=\int a_{0} d a_{1}$ of the cycle $\left(\Omega, d, \int\right)$ is equal to the cocycle

$$
\psi_{1}\left(a_{0}, a_{1}\right)=2 f a_{0} \delta\left(a_{1}\right) P|D|^{-1}-f a_{0} \delta^{2}\left(a_{1}\right) P|D|^{-1},
$$

with $P=(1+F) / 2$. The local index formula is given by

$$
\varphi_{\text {odd }}=\psi_{1}+(b+B) \varphi_{\text {even }},
$$

where $\varphi$ is the local index cocycle.

(4) The character $\operatorname{Tr}\left(a_{0}\left[F, a_{1}\right]\right)$ differs from the local form $\psi_{1}$ by the coboundary b $\psi_{0}$, with $\psi_{0}(a)=2 \operatorname{Tr}\left(a P|D|^{-s}\right)_{s=0}$. This cochain is determined by the values $\psi_{0}\left(\left(\beta^{*} \beta\right)^{n}\right)$, which are of the form

$$
\psi_{0}\left(\left(\beta^{*} \beta\right)^{n}\right)=q^{-2 n}\left(q^{2} R_{n}\left(q^{2}\right)-G\left(q^{2}\right)\right),
$$

where $G$ is the logarithmic derivative of the Dedekind eta function

$$
\eta\left(q^{2}\right)=q^{1 / 12} \prod_{k=1}^{\infty}\left(1-q^{2 k}\right),
$$

and the $R_{n}$ are rational functions with poles only at roots of unity.

More recently, another important breakthrough in the relation between quantum groups and the formalism of spectral triples was obtained by Walter van Suijlekom, Ludwik Dabrowski, Giovanni Landi, Andrzej Sitarz, Joseph C. Varilly, in [191] and 192.

They construct a $3^{+}$summable spectral triple $(\mathcal{A}, \mathcal{H}, D)$, where $\mathcal{A}$ is, as before, the algebra of coordinates of the quantum group $S U_{q}(2)$. The geometry in this case is an isospectral deformation of the classical case, in the sense that the Dirac operator is the same as the usual Dirac operator for the round metric on the ordinary 3 -sphere $S^{3}$. Moreover, the spectral triple $(\mathcal{A}, \mathcal{H}, D)$ is especially nice, in as it is equivariant with respect to both left and right action of the Hopf algebra $\mathcal{U}_{q}\left(s u_{q}(2)\right)$.

The classical Dirac operator for the round metric on $S^{3}$ has spectrum $\Sigma=\Sigma_{+} \cup \Sigma_{-}$with $\Sigma_{+}=\{(2 j+3 / 2): j=0,1 / 2,1,3 / 2, \ldots\}$ with multiplicities $(2 j+1)(2 j+2)$ and $\Sigma_{-}=$ $\{-(2 j+1 / 2): j=1 / 2,1,3 / 2, \ldots\}$ with multiplicities $2 j(2 j+1)$. The Hilbert space is obtained by taking $V \otimes \mathbb{C}^{2}$, where $V$ is the left regular representation of $\mathcal{A}$. It is very important here to take $V \otimes \mathbb{C}^{2}$ instead of $\mathbb{C}^{2} \otimes V$. Not only the latter violates the equivariance condition, but it was shown by Ghoswami that it produces unbounded commutators $[D, a]$, hence one does not obtain a spectral triple in that way.

The spectral triple contructed in [191] and [192] has a real structure $J$ and the Dirac operator satisfies a weak form of the "order one condition" ( $c f$. Section 13 above). The local index formula of 65] ( $c f$. Theorem 20.2 above) extends to the spectral triple of [191], as proved in [192] and the structures of the cotangent space and the geodesic flow are essentially the same. 


\section{SPHERICAL MANIFOLDS}

The noncommutative spheres $S_{\varphi}^{3} \subset \mathbb{R}_{\varphi}^{4}$ are obtained as solutions of a very simple problem, namely the vanishing of the first component of the Chern character of a unitary $U \in M_{2}(\mathcal{A})$ where $\mathcal{A}$ is the algebra of functions on the sphere and the Chern character is taken in the cyclic homology $(\mathrm{b}, \mathrm{B})$ bicomplex. The origin of this problem is to quantize the volume form of a three manifold ( $c f$. 69]). The solutions are parameterized by three angles $\varphi_{k}, k \in\{1,2,3\}$ and the corresponding algebras are obtained by imposing the "unit sphere relation"

$$
\sum x_{\mu}^{2}=1
$$

to the four generators $x_{0}, x_{1}, x_{2}, x_{3}$ of the quadratic algebra $C_{\mathrm{alg}}\left(\mathbb{R}_{\varphi}^{4}\right)$ with the six relations

$$
\begin{aligned}
& \sin \left(\varphi_{k}\right)\left[x_{0}, x_{k}\right]_{+}=i \cos \left(\varphi_{\ell}-\varphi_{m}\right)\left[x_{\ell}, x_{m}\right] \\
& \cos \left(\varphi_{k}\right)\left[x_{0}, x_{k}\right]=i \sin \left(\varphi_{\ell}-\varphi_{m}\right)\left[x_{\ell}, x_{m}\right]_{+},
\end{aligned}
$$

where $[a, b]_{+}=a b+b a$ is the anticommutator and by convention the indices $k, l, m \in\{1,2,3\}$ always appear in cyclic order.

The analysis of these algebras is a special case of the general theory of central quadratic forms for quadratic algebras developed in [70, 71] and which we briefly recall below.

Let $\mathcal{A}=A(V, R)=T(V) /(R)$ be a quadratic algebra where $V$ is the linear span of the generators and $(R) \subset T(V)$ the ideal generated by the relations. The geometric data $\{E, \sigma, \mathcal{L}\}$ is given by an algebraic variety $E$, a correspondence $\sigma$ on $E$ and a line bundle $\mathcal{L}$ over $E$. These data are defined so as to yield an homomorphism $h$ from $\mathcal{A}$ to a crossed product algebra constructed from sections of powers of the line bundle $\mathcal{L}$ on the graphs of the iterations of the correspondence $\sigma$. This crossed product only involves the positive powers of the correspondence $\sigma$ and thus remains "triangular" and far remote from the "semi-simple" set-up of $C^{*}$-algebras.

This morphism $h$ can be considerably refined using the notion of positive central quadratic form.

Definition 21.1. Let $Q \in S^{2}(V)$ be a symmetric bilinear form on $V^{*}$ and $C$ a component of $E \times E$. We say that $Q$ is central on $C$ iff for all $\left(Z, Z^{\prime}\right)$ in $C$ and $\omega \in R$ one has,

$$
\omega\left(Z, Z^{\prime}\right) Q\left(\sigma\left(Z^{\prime}\right), \sigma^{-1}(Z)\right)+Q\left(Z, Z^{\prime}\right) \omega\left(\sigma\left(Z^{\prime}\right), \sigma^{-1}(Z)\right)=0
$$

This makes it possible to construct purely algebraically a crossed product algebra and an homomorphism from $\mathcal{A}=A(V, R)$ to this crossed product [0], 71]. The relation with $C^{*}$ algebras arises from positive central quadratic forms which make sense on involutive quadratic algebras.

Let $\mathcal{A}=A(V, R)$ be an involutive quadratic algebra i.e. an algebra over $\mathbb{C}$ which is a $*$-algebra with involution $x \mapsto x^{*}$ preserving the subspace $V$ of the generators. The real structure of $V$ is given by the antilinear involution $v \mapsto j(v)$ restriction of $x \mapsto x^{*}$. As $(x y)^{*}=y^{*} x^{*}$ for $x, y \in \mathcal{A}$, the space $R$ of relations fulfills

$$
(j \otimes j)(R)=t(R)
$$


in $V \otimes V$ where $t: V \otimes V \rightarrow V \otimes V$ is the transposition $v \otimes w \mapsto t(v \otimes w)=w \otimes v$. This implies that the characteristic variety is stable under the involution $j$ and one has

$$
\sigma(j(Z))=j\left(\sigma^{-1}(Z)\right)
$$

Let then $C$ be an invariant component of $E \times E$, we say that $C$ is $j$-real when it is globally invariant under the involution

$$
\tilde{j}\left(Z, Z^{\prime}\right):=\left(j\left(Z^{\prime}\right), j(Z)\right)
$$

Let then $Q$ be a central qudratic form on $C$, we say that $Q$ is positive on $C$ iff

$$
Q(Z, j(Z))>0, \quad \forall Z \in K
$$

One can then endow the line bundle $\mathcal{L}$ dual of the tautological bundle on $P\left(V^{*}\right)$ with the hermitian metric given by

$$
\left\langle f L, g L^{\prime}\right\rangle_{Q}(Z)=f(Z) \overline{g(Z)} \frac{L(Z) \overline{L^{\prime}(Z)}}{Q(Z, j(Z))} \quad L, L^{\prime} \in V, \quad Z \in K .
$$

$(\forall f, g \in C(K))$

One then defines a generalized crossed product $C^{*}$-algebra $C(K) \times_{\sigma, \mathcal{L}} \mathbb{Z}$ following M. Pimsner 166. Given a compact space $K$, an homeomorphism $\sigma$ of $K$ and a hermitian line bundle $\mathcal{L}$ on $K$ we define the $C^{*}$-algebra $C(K) \times_{\sigma, \mathcal{L}} \mathbb{Z}$ as the twisted cross-product of $C(K)$ by the Hilbert $C^{*}$-bimodule associated to $\mathcal{L}$ and $\sigma$ ([3], [166]).

We let for each $n \geq 0, \mathcal{L}^{\sigma^{n}}$ be the hermitian line bundle pullback of $\mathcal{L}$ by $\sigma^{n}$ and (cf. [10, [188])

$$
\mathcal{L}_{n}:=\mathcal{L} \otimes \mathcal{L}^{\sigma} \otimes \cdots \otimes \mathcal{L}^{\sigma^{n-1}}
$$

We first define a $*$-algebra as the linear span of the monomials

$$
\xi W^{n}, \quad W^{* n} \eta^{*}, \quad \xi, \eta \in C\left(K, \mathcal{L}_{n}\right)
$$

with product given as in ([10, [188]) for $\left(\xi_{1} W^{n_{1}}\right)\left(\xi_{2} W^{n_{2}}\right)$ so that

$$
\left(\xi_{1} W^{n_{1}}\right)\left(\xi_{2} W^{n_{2}}\right):=\left(\xi_{1} \otimes\left(\xi_{2} \circ \sigma^{n_{1}}\right)\right) W^{n_{1}+n_{2}}
$$

We use the hermitian structure of $\mathcal{L}_{n}$ to give meaning to the products $\eta^{*} \xi$ and $\xi \eta^{*}$ for $\xi, \eta \in C\left(K, \mathcal{L}_{n}\right)$. The product then extends uniquely to an associative product of $*$-algebra fulfilling the following additional rules

$$
\left(W^{* k} \eta^{*}\right)\left(\xi W^{k}\right):=\left(\eta^{*} \xi\right) \circ \sigma^{-k}, \quad\left(\xi W^{k}\right)\left(W^{* k} \eta^{*}\right):=\xi \eta^{*}
$$

The $C^{*}$-norm of $C(K) \times_{\sigma, \mathcal{L}} \mathbb{Z}$ is defined as for ordinary cross-products and due to the amenability of the group $\mathbb{Z}$ there is no distinction between the reduced and maximal norms. The latter is obtained as the supremum of the norms in involutive representations in Hilbert space. The natural positive conditional expectation on the subalgebra $C(K)$ shows that the $C^{*}$-norm restricts to the usual sup norm on $C(K)$. 
Theorem 21.2. Let $K \subset E$ be a compact $\sigma$-invariant subset and $Q$ be central and strictly positive on $\{(Z, \bar{Z}) ; Z \in K\}$. Let $\mathcal{L}$ be the restriction to $K$ of the dual of the tautological line bundle on $P\left(V^{*}\right)$ endowed with the hermitian metric $\langle,\rangle_{Q}$.

(i) The equality $\sqrt{2} \theta(Y):=Y W+W^{*} \bar{Y}^{*}$ yields a $*$-homomorphism

$$
\theta: \mathcal{A}=A(V, R) \rightarrow C(K) \times_{\sigma, \mathcal{L}} \mathbb{Z}
$$

(ii) For any $Y \in V$ the $C^{*}$-norm of $\theta(Y)$ fulfills

$$
\sup _{K}\|Y\| \leq \sqrt{2}\|\theta(Y)\| \leq 2 \sup _{K}\|Y\|
$$

(iii) If $\sigma^{4} \neq \mathbb{1}$, then $\theta(Q)=1$ where $Q$ is viewed as an element of $T(V) /(R)$.

In the above case of the sphere $S_{\varphi}^{3}$ one lets $Q$ be the quadratic form

$$
Q\left(x, x^{\prime}\right):=\sum x_{\mu} x_{\mu}^{\prime}
$$

In the generic case one has :

Proposition 21.3. 1) The characteristic variety is the union of 4 points with an elliptic curve $F_{\varphi}$.

2) The quadratic form $Q$ is central and positive on $F_{\varphi} \times F_{\varphi}$.

In suitable coordinates the equations defining the elliptic curve $F_{\varphi}$ are

$$
\frac{Z_{0}^{2}-Z_{1}^{2}}{s_{1}}=\frac{Z_{0}^{2}-Z_{2}^{2}}{s_{2}}=\frac{Z_{0}^{2}-Z_{3}^{2}}{s_{3}}
$$

where $s_{k}:=1+t_{\ell} t_{m}, t_{k}:=\tan \varphi_{k}$.

The positivity of $Q$ is automatic since in the coordinates $x$ the involution $j_{\varphi}$ of the $*$-algebra $C_{\text {alg }}\left(\mathbb{R}_{\varphi}^{4}\right)$ is just $j_{\varphi}(Z)=\bar{Z}$, so that $Q\left(X, j_{\varphi}(X)\right)>0$ for $X \neq 0$.

Corollary 21.4. Let $K \subset F_{\varphi}$ be a compact $\sigma$-invariant subset. The homomorphism $\theta$ of Theorem 21.2 is a unital *-homomorphism from $C_{\mathrm{alg}}\left(S_{\varphi}^{3}\right)$ to the cross-product $C^{\infty}(K) \times_{\sigma, \mathcal{L}} \mathbb{Z}$.

It follows that one obtains a non-trivial $C^{*}$-algebra $C^{*}\left(S_{\varphi}^{3}\right)$ as the completion of $C_{\text {alg }}\left(S_{\varphi}^{3}\right)$ for the semi-norm,

$$
\|P\|:=\sup \|\pi(P)\|
$$

where $\pi$ varies through all unitary representations of $C_{\mathrm{alg}}\left(S_{\varphi}^{3}\right)$. It was clear from the start that (21.14) defines a finite $C^{*}$-semi-norm on $C_{\text {alg }}\left(S_{\varphi}^{3}\right)$ since the equation of the sphere $\sum x_{\mu}^{2}=1$ together with the self-adjointness $x_{\mu}=x_{\mu}^{*}$ show that in any unitary representation one has

$$
\left\|\pi\left(x_{\mu}\right)\right\| \leq 1, \quad \forall \mu .
$$

What the above corollary gives is a lower bound for the $C^{*}$-norm such as that given by statement (ii) of Theorem 21.2 on the linear subspace $V$ of generators.

The correspondence $\sigma$ on $F_{\varphi}$, is for generic $\varphi$ a translation of module $\eta$ of the elliptic curve $F_{\varphi}$ and one distinguishes two cases : the even case when it preserves the two real components of the curve $F_{\varphi} \cap P_{3}(\mathbb{R})$ and the odd case when it permutes them. 
Proposition 21.5. Let $\varphi$ be generic and even.

(i) The cross-product $C\left(F_{\varphi}\right) \times_{\sigma, \mathcal{L}} \mathbb{Z}$ is isomorphic to the mapping torus of the automorphism $\beta$ of the noncommutative torus $\mathbb{T}_{\eta}^{2}=C_{\varphi} \times_{\sigma} \mathbb{Z}$ acting on the generators by the matrix $\left[\begin{array}{ll}1 & 4 \\ 0 & 1\end{array}\right]$.

(ii) The crossed product $F_{\varphi} \times_{\sigma, \mathcal{L}} \mathbb{Z}$ is a noncommutative 3 -manifold with an elliptic action of the three dimensional Heisenberg Lie algebra $\mathfrak{h}_{3}$ and an invariant trace $\tau$.

It follows that one is exactly in the framework developed in [57. We refer to [176] and [2] where these noncommutative manifolds were analyzed in terms of crossed products by Hilbert $C^{*}$-bimodules.

Integration on the translation invariant volume form $d v$ of $F_{\varphi}$ gives the $\mathfrak{h}_{3}$-invariant trace $\tau$,

$$
\begin{aligned}
\tau(f) & =\int f d v, \quad \forall f \in C^{\infty}\left(F_{\varphi}\right) \\
\tau\left(\xi W^{k}\right) & =\tau\left(W^{* k} \eta^{*}\right)=0, \quad \forall k \neq 0
\end{aligned}
$$

It follows in particular that the results of [57] apply to obtain the calculus. In particular the following gives the "fundamental class" as a 3-cyclic cocycle,

$$
\tau_{3}\left(a_{0}, a_{1}, a_{2}, a_{3}\right)=\sum \epsilon_{i j k} \tau\left(a_{0} \delta_{i}\left(a_{1}\right) \delta_{j}\left(a_{2}\right) \delta_{k}\left(a_{3}\right)\right)
$$

where the $\delta_{j}$ are the generators of the action of $\mathfrak{h}_{3}$.

The relation between the noncommutative spheres $S_{\varphi}^{3}$ and the noncommutative nilmanifolds $F_{\varphi} \times_{\sigma, \mathcal{L}} \mathbb{Z}$ is analyzed in [70, [7] thanks to the computation of the Jacobian of the homomorphism $\theta$.

\section{2. $\mathbb{Q}$-LATtices}

A class of examples of noncommutative spaces of relevance to number theory is given by the moduli spaces of $\mathbb{Q}$-lattices up to commensurability. These fall within the general framework of noncommutative spaces obtained as quotients of equivalence relations discussed in Section 4.

A $\mathbb{Q}$-lattice in $\mathbb{R}^{n}$ consists of a pair $(\Lambda, \phi)$ of a lattice $\Lambda \subset \mathbb{R}^{n}$ (a cocompact free abelian subgroup of $\mathbb{R}^{n}$ of rank $n$ ) together with a system of labels of its torsion points given by a homomorphism of abelian groups

$$
\phi: \mathbb{Q}^{n} / \mathbb{Z}^{n} \longrightarrow \mathbb{Q} \Lambda / \Lambda
$$

Two $\mathbb{Q}$-lattices are commensurable,

$$
\left(\Lambda_{1}, \phi_{1}\right) \sim\left(\Lambda_{2}, \phi_{2}\right)
$$

iff $\mathbb{Q} \Lambda_{1}=\mathbb{Q} \Lambda_{2}$ and

$$
\phi_{1}=\phi_{2} \bmod \Lambda_{1}+\Lambda_{2}
$$

In general, the map $\phi$ of (22.1) is just a group homomorphism. A $\mathbb{Q}$-lattice is said to be invertible is $\phi$ is an isomorphism. Two invertible $\mathbb{Q}$-lattices are commensurable if and only if they are equal. 


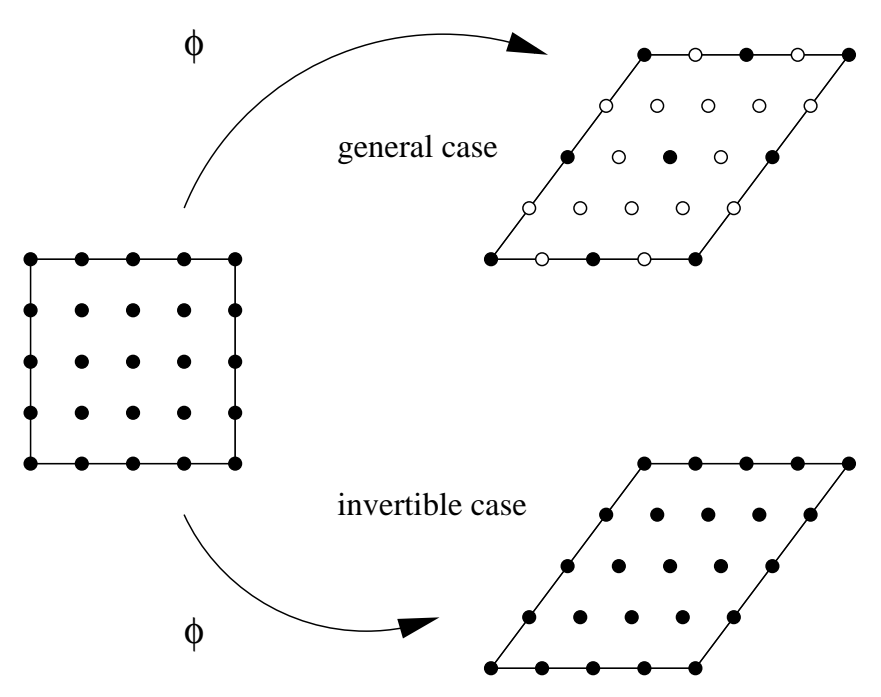

FiguRE 15. $\mathbb{Q}$-lattices: generic and invertible case.

The space $\mathcal{L}_{n}$ of commensurabilty classes of $\mathbb{Q}$-lattices in $\mathbb{R}^{n}$ has the typical property of noncommutative spaces: it has the cardinality of the continuum but one cannot construct a countable collection of measurable functions that separate points of $\mathcal{L}_{n}$. Thus, one can use noncommutative geometry to describe the quotient space $\mathcal{L}_{n}$ through a noncommutative $C^{*}$-algebra $C^{*}\left(\mathcal{L}_{n}\right)$.

We consider especially the case of $n=1$ and $n=2$. One is also interested in the $C^{*}$-algebras describing $\mathbb{Q}$-lattices up to scaling, $\mathcal{A}_{1}=C^{*}\left(\mathcal{L}_{1} / \mathbb{R}_{+}^{*}\right)$ and $\mathcal{A}_{2}=C^{*}\left(\mathcal{L}_{2} / \mathbb{C}^{*}\right)$.

In the 1-dimensional case, a $\mathbb{Q}$-lattice can always be written in the form

$$
(\Lambda, \phi)=(\lambda \mathbb{Z}, \lambda \rho)
$$

for some $\lambda>0$ and some

$$
\rho \in \operatorname{Hom}(\mathbb{Q} / \mathbb{Z}, \mathbb{Q} / \mathbb{Z})=\lim _{\longleftarrow} \mathbb{Z} / n \mathbb{Z}=\hat{\mathbb{Z}}
$$

By considering lattices up to scaling, we eliminate the factor $\lambda>0$ so that 1-dimensional $\mathbb{Q}$-lattices up to scale are completely specified by the choice of the element $\rho \in \hat{\mathbb{Z}}$. Thus, the algebra of coordinates of the space of 1 -dimensional $\mathbb{Q}$-lattices up to scale is the commutative $C^{*}$-algebra

$$
C(\hat{\mathbb{Z}}) \simeq C^{*}(\mathbb{Q} / \mathbb{Z})
$$

where we use Pontrjagin duality to get the identification in (22.4).

The equivalence relation of commensurability is implemented by the action of the semigroup $\mathbb{N}^{\times}$on $\mathbb{Q}$-lattices. The corresponding action on the algebra (22.4) is by

$$
\alpha_{n}(f)(\rho)=\left\{\begin{array}{lr}
f\left(n^{-1} \rho\right) & \rho \in n \hat{\mathbb{Z}} \\
0 & \text { otherwise. }
\end{array}\right.
$$

Thus, the quotient of the space of 1-dimensional $\mathbb{Q}$-lattices up to scale by the commensurability relation and its algebra of coordinates is given by the semigroup crossed product 


$$
C^{*}(\mathbb{Q} / \mathbb{Z}) \rtimes \mathbb{N}^{\times}
$$

This is the Bost-Connes $C^{*}$-algebra introduced in [27].

It has a natural time evolution given by the covolume of a pair of commensurable $\mathbb{Q}$-lattices. It has symmetries (compatible with the time evolution) given by the group $\hat{\mathbb{Z}}^{*}=\mathrm{GL}_{1}\left(\mathbb{A}_{f}\right) / \mathbb{Q}^{*}$ and the KMS (Kubo-Martin-Schwinger) equilibrium states of the system have interesting arithmetic properties. Namely, the partition function of the system is the Riemann zeta function. There is a unique KMS state for sufficiently high temperature, while at low temperature the system undergoes a phase transition with spontaneous symmetry breaking. The pure phases (estremal KMS states) at low temperature are parameterized by elements in $\hat{\mathbb{Z}}^{*}$. They have an explicit expression in terms of polylogarithms at roots of unity. At zero temperature the extremal KMS states, evaluated on the elements of a rational subalgebra affect values that are algebraic numbers. The action on these values of the Galois group $\operatorname{Gal}(\overline{\mathbb{Q}} / \mathbb{Q})$ factors through its abelianization and is obtained (via the class field theory isomorphism $\left.\hat{\mathbb{Z}}^{*} \cong \operatorname{Gal}\left(\mathbb{Q}^{a b} / \mathbb{Q}\right)\right)$ as the action of symmetries on the algebra (cf. [27], 74], 75] for details).

In the 2-dimensional case, a $\mathbb{Q}$-lattice can be written in the form

$$
(\Lambda, \phi)=(\lambda(\mathbb{Z}+\mathbb{Z} \tau), \lambda \rho)
$$

for some $\lambda \in \mathbb{C}^{*}$, some $\tau \in \mathbb{H}$, and some $\rho \in M_{2}(\hat{\mathbb{Z}})=\operatorname{Hom}\left(\mathbb{Q}^{2} / \mathbb{Z}^{2}, \mathbb{Q}^{2} / \mathbb{Z}^{2}\right)$. Thus, the space of 2-dimensional $\mathbb{Q}$-lattices up to the scale factor $\lambda \in \mathbb{C}^{*}$ and up to isomorphisms, is given by

$$
M_{2}(\hat{\mathbb{Z}}) \times \mathbb{H} \quad \bmod \Gamma=\mathrm{SL}_{2}(\mathbb{Z}) .
$$

The commensurability relation giving the space $\mathcal{L}_{2} / \mathbb{C}^{*}$ is implemented by the partially defined action of $\mathrm{GL}_{2}^{+}(\mathbb{Q})$.

One considers in this case the quotient of the space

$$
\tilde{\mathcal{U}}:=\left\{(g, \rho, \alpha) \in \mathrm{GL}_{2}^{+}(\mathbb{Q}) \times M_{2}(\hat{\mathbb{Z}}) \times \mathrm{GL}_{2}^{+}(\mathbb{R}): g \rho \in M_{2}(\hat{\mathbb{Z}})\right\}
$$

by the action of $\Gamma \times \Gamma$ given by

$$
\left(\gamma_{1}, \gamma_{2}\right)(g, \rho, \alpha)=\left(\gamma_{1} g \gamma_{2}^{-1}, \gamma_{2} \rho, \gamma_{2} \alpha\right)
$$

The groupoid $\mathcal{R}_{2}$ of the equivalence relation of commensurability on 2-dimensional $\mathbb{Q}$-lattices (not considered up to scaling for the moment) is a locally compact groupoid, which can be parameterized by the quotient of (22.8) by $\Gamma \times \Gamma$ via the map $r: \tilde{\mathcal{U}} \rightarrow \mathcal{R}_{2}$,

$$
r(g, \rho, \alpha)=\left(\left(\alpha^{-1} g^{-1} \Lambda_{0}, \alpha^{-1} \rho\right),\left(\alpha^{-1} \Lambda_{0}, \alpha^{-1} \rho\right)\right) .
$$

We then consider the quotient by scaling. The quotient $\mathrm{GL}_{2}^{+}(\mathbb{R}) / \mathbb{C}^{*}$ can be identified with the hyperbolic plane $\mathbb{H}$ in the usual way. If $\left(\Lambda_{k}, \phi_{k}\right) k=1,2$ are a pair of commensurable 2-dimensional $\mathbb{Q}$-lattices, then for any $\lambda \in \mathbb{C}^{*}$, the $\mathbb{Q}$-lattices $\left(\lambda \Lambda_{k}, \lambda \phi_{k}\right)$ are also commensurable, with

$$
r\left(g, \rho, \alpha \lambda^{-1}\right)=\lambda r(g, \rho, \alpha) .
$$

However, the action of $\mathbb{C}^{*}$ on $\mathbb{Q}$-lattices is not free due to the presence of lattices (such as $\Lambda_{0}$ above) with nontrivial automorphisms. Thus, the quotient $Z=\mathcal{R}_{2} / \mathbb{C}^{*}$ is no longer a groupoid. Still, one can define a convolution algebra for $Z$ by restricting the convolution 
product of $\mathcal{R}_{2}$ to homogeneous functions of weight zero, where a function $f$ has weight $k$ if it satisfies

$$
f(g, \rho, \alpha \lambda)=\lambda^{k} f(g, \rho, \alpha), \quad \forall \lambda \in \mathbb{C}^{*} .
$$

The space $Z$ is the quotient of the space

$$
\mathcal{U}:=\left\{(g, \rho, z) \in \mathrm{GL}_{2}^{+}(\mathbb{Q}) \times M_{2}(\hat{\mathbb{Z}}) \times \mathbb{H} \mid g \rho \in M_{2}(\hat{\mathbb{Z}})\right\}
$$

by the action of $\Gamma \times \Gamma$. Here the space $M_{2}(\hat{\mathbb{Z}}) \times \mathbb{H}$ has a partially defined action of $\mathrm{GL}_{2}^{+}(\mathbb{Q})$ given by

$$
g(\rho, z)=(g \rho, g(z))
$$

where $g(z)$ denotes action as fractional linear transformation.

Thus, the algebra of coordinates $\mathcal{A}_{2}$ for the noncommutative space of commensurability classes of 2-dimensional $\mathbb{Q}$-lattices up to scaling is given by the following convolution algebra. Consider the space $C_{c}(Z)$ of continuous compactly supported functions on $Z$. These can be seen, equivalently, as functions on $\mathcal{U}$ as in (22.11) invariant under the $\Gamma \times \Gamma$ action $(g, \rho, z) \mapsto$ $\left(\gamma_{1} g \gamma_{2}^{-1}, \gamma_{2} z\right)$. One endows $C_{c}(Z)$ with the convolution product

$$
\left(f_{1} * f_{2}\right)(g, \rho, z)=\sum_{s \in \Gamma \backslash \mathrm{GL}_{2}^{+}(\mathbb{Q}): s \rho \in M_{2}(\hat{\mathbb{Z}})} f_{1}\left(g s^{-1}, s \rho, s(z)\right) f_{2}(s, \rho, z)
$$

and the involution $f^{*}(g, \rho, z)=\overline{f\left(g^{-1}, g \rho, g(z)\right)}$.

Again there is a time evolution on this algebra, which is given by the covolume,

$$
\sigma_{t}(f)(g, \rho, z)=\operatorname{det}(g)^{i t} f(g, \rho, z) \text {. }
$$

The partition function for this $\mathrm{GL}_{2}$ system is given by

$$
Z(\beta)=\sum_{m \in \Gamma \backslash M_{2}^{+}(\mathbb{Z})} \operatorname{det}(m)^{-\beta}=\sum_{k=1}^{\infty} \sigma(k) k^{-\beta}=\zeta(\beta) \zeta(\beta-1),
$$

where $\sigma(k)=\sum_{d \mid k} d$. The form of the partition function suggests the possibility that two distinct phase transitions might happen at $\beta=1$ and $\beta=2$.

The structure of KMS states for this system is analysed in [74. The main result is the following.

Theorem 22.1. The $K M S_{\beta}$ states of the $\mathrm{GL}_{2}$-system have the following properties:

(1) In the range $\beta \leq 1$ there are no KMS states.

(2) In the range $\beta>2$ the set of extremal KMS states is given by the classical Shimura variety

$$
\mathcal{E}_{\beta} \cong \mathrm{GL}_{2}(\mathbb{Q}) \backslash \mathrm{GL}_{2}(\mathbb{A}) / \mathbb{C}^{*}
$$

The symmetries are more complicated than in the Bost-Connes case. In fact, in addition to symmetries given by automorphisms that commute with the time evolution, there are also symmetries by endomorphisms that play an important role. The resulting symmetry group is the quotient $\mathrm{GL}_{2}\left(\mathbb{A}_{f}\right) / \mathbb{Q}^{*}$. An important result of Shimura 184. shows that this group is in fact the Galois group of the field $F$ of modular functions. The group $\mathrm{GL}_{2}\left(\mathbb{A}_{f}\right)$ decomposes as a product

$$
\mathrm{GL}_{2}\left(\mathbb{A}_{f}\right)=\mathrm{GL}_{2}^{+}(\mathbb{Q}) \mathrm{GL}_{2}(\hat{\mathbb{Z}})
$$


where $\mathrm{GL}_{2}(\hat{\mathbb{Z}})$ acts by automorphisms related to the deck transformations of the tower of the modular curves, while $\mathrm{GL}_{2}^{+}(\mathbb{Q})$ acts by endomorphisms that move across levels in the modular tower.

The modular field $F$ is the field of modular functions over $\mathbb{Q}^{a b}$, namely the union of the fields $F_{N}$ of modular functions of level $N$ rational over the cyclotomic field $\mathbb{Q}\left(\zeta_{n}\right)$, that is, such that the $q$-expansion in powers of $q^{1 / N}=\exp (2 \pi i \tau / N)$ has all coefficients in $\mathbb{Q}\left(e^{2 \pi i / N}\right)$.

The action of the Galois group $\hat{\mathbb{Z}}^{*} \simeq \operatorname{Gal}\left(\mathbb{Q}^{a b} / \mathbb{Q}\right)$ on the coefficients determines a homomorphism

$$
\operatorname{cycl}: \hat{\mathbb{Z}}^{*} \rightarrow \operatorname{Aut}(F) \text {. }
$$

If $\tau \in \mathbb{H}$ is a generic point, then the evaluation map $f \mapsto f(\tau)$ determines an embedding $F \hookrightarrow \mathbb{C}$. We denote by $F_{\tau}$ the image in $\mathbb{C}$. This yields an identification

$$
\theta_{\tau}: \operatorname{Gal}\left(F_{\tau} / \mathbb{Q}\right) \stackrel{\simeq}{\rightarrow} \mathbb{Q}^{*} \backslash \mathrm{GL}_{2}\left(\mathbb{A}_{f}\right) .
$$

There is an arithmetic algebra $\mathcal{A}_{2, \mathbb{Q}}($ defined over $\mathbb{Q})$ of unbounded multipliers of the $C^{*}$ algebra $\mathcal{A}_{2}$, obtained by considering continuous functions on $Z$ (cf. (22.11)), with finite support in the variable $g \in \Gamma \backslash \mathrm{GL}_{2}^{+}(\mathbb{Q})$ and with the following properties. Let $p_{N}: M_{2}(\hat{\mathbb{Z}}) \rightarrow$ $M_{2}(\mathbb{Z} / N \mathbb{Z})$ be the canonical projection. With the notation $f_{(g, \rho)}(z)=f(g, \rho, z)$, we say that $f_{(g, \rho)} \in C(\mathbb{H})$ is of level $N$ if

$$
f_{(g, \rho)}=f_{\left(g, p_{N}(\rho)\right)} \quad \forall(g, \rho) .
$$

We require that elements of $\mathcal{A}_{2, \mathbb{Q}}$ have the $f_{(g, \rho)}$ of finite level with $f_{(g, m)} \in F$ for all $(g, m)$. We also require that the action (22.17) on the coefficients of the q-expansion of the $f_{(g, m)}$ satisfies

$$
f_{(g, \alpha(u) m)}=\operatorname{cycl}(u) f_{(g, m)},
$$

for all $g \in \mathrm{GL}_{2}^{+}(\mathbb{Q})$ diagonal and all $u \in \hat{\mathbb{Z}}^{*}$, with

$$
\alpha(u)=\left(\begin{array}{ll}
u & 0 \\
0 & 1
\end{array}\right),
$$

to avoid some "trivial" elements that would spoil the Galois action on values of states ( $c f$. [74], 75]). The action of symmetries extends to $\mathcal{A}_{2, \mathbb{Q}}$. We have then the following result $(74)$ :

Theorem 22.2. Consider a state $\varphi=\varphi_{\infty, L} \in \mathcal{E}_{\infty}$, for a generic invertible $\mathbb{Q}$-lattice $L=$ $(\rho, \tau)$. Then the values of the state on elements of the arithmetic subalgebra generate the image in $\mathbb{C}$ of the modular field,

$$
\varphi\left(\mathcal{A}_{2, \mathbb{Q}}\right) \subset F_{\tau},
$$

and the isomorphism

$$
\theta_{\varphi}: \operatorname{Gal}\left(F_{\tau} / \mathbb{Q}\right) \stackrel{\simeq}{\longrightarrow} \mathbb{Q}^{*} \backslash \mathrm{GL}_{2}\left(\mathbb{A}_{f}\right),
$$

given by

$$
\theta_{\varphi}(\gamma)=\rho^{-1} \theta_{\tau}(\gamma) \rho
$$


for $\theta_{\tau}$ as in (22.18), intertwines the Galois action on the values of the state with the action of symmetries,

$$
\gamma \varphi(f)=\varphi\left(\theta_{\varphi}(\gamma) f\right), \quad \forall f \in \mathcal{A}_{2, \mathbb{Q}}, \quad \forall \gamma \in \operatorname{Gal}\left(F_{\tau} / \mathbb{Q}\right)
$$

A notion analogous to that of $\mathbb{Q}$-lattices can be given for other number fields $\mathbb{K}$. This notion was used in [78] to construct a quantum statistical mechanical system for $\mathbb{K}$ an imaginary quadratic field. This system shares properties with both the Bost-Connes system of [27] and the $\mathrm{GL}_{2}$ system (2-dimensional $\mathbb{Q}$-lattices) of 74 .

We assume that $\mathbb{K}=\mathbb{Q}(\sqrt{-d}), d$ a positive integer. Let $\tau \in \mathbb{H}$ be such that $\mathbb{K}=\mathbb{Q}(\tau)$ and $\mathcal{O}=\mathbb{Z}+\mathbb{Z} \tau$ is the ring of integers of $\mathbb{K}$.

A 1 -dimensional $\mathbb{K}$-lattice $(\Lambda, \phi)$ is a finitely generated $\mathcal{O}_{\text {-submodule }} \Lambda \subset \mathbb{C}$, such that $\Lambda \otimes_{\mathcal{O}}$ $\mathbb{K} \cong \mathbb{K}$, together with a morphism of $\mathcal{O}$-modules

$$
\phi: \mathbb{K} / \mathcal{O} \rightarrow \mathbb{K} \Lambda / \Lambda \text {. }
$$

A 1-dimensional $\mathbb{K}$-lattice is invertible if $\phi$ is an isomorphism of $\mathcal{O}$-modules. A 1-dimensional $\mathbb{K}$-lattice is, in particular, a 2-dimensional $\mathbb{Q}$-lattice.

We consider the notion of commensurability as in the case of $\mathbb{Q}$-lattices. Two 1-dimensional $\mathbb{K}$-lattices $\left(\Lambda_{1}, \phi_{1}\right)$ and $\left(\Lambda_{2}, \phi_{2}\right)$ are commensurable if $\mathbb{K} \Lambda_{1}=\mathbb{K} \Lambda_{2}$ and $\phi_{1}=\phi_{2}$ modulo $\Lambda_{1}+\Lambda_{2}$. In particular, two 1-dimensional $\mathbb{K}$-lattices are commensurable iff the underlying $\mathbb{Q}$-lattices are commensurable.

The algebra of the corresponding noncommutative space is a restriction of the algebra of the $\mathrm{GL}_{2}$-system to the subgroupoid of the equivalence of commensurability restricted to $\mathbb{K}$ lattices. The time evolution is also a restriction from the $\mathrm{GL}_{2}$-system.

The resulting system has partition function the Dedekind zeta function $\zeta_{\mathbb{K}}(\beta)$ of the number field $\mathbb{K}$. Above the critical temperature $T=1$ there is a unique KMS state, while at lower temperatures the extremal KMS states are parameterized by elements of $\mathbb{A}_{\mathbb{K}}^{*} / \mathbb{K}^{*}$, where $\mathbb{A}_{K}=\mathbb{A}_{K, f} \times \mathbb{C}$ are the adeles of $\mathbb{K}$, with $\mathbb{A}_{K, f}=\mathbb{A}_{f} \otimes \mathbb{K}$. The KMS states at zero temperature, evaluated on the restriction to $\mathbb{K}$-lattices of the arithmetic algebra of the $\mathrm{GL}_{2}$-system, have an action of the Galois group $\operatorname{Gal}\left(\mathbb{K}^{a b} / \mathbb{K}\right)$ realized (via the class field theory isomorphism) through the action of symmetries (automorphisms and endomorphisms) of the system ( $c f$. [78).

\section{Modular Hecke algebras}

Connes and Moscovici [84] defined modular Hecke algebras $\mathcal{A}(\Gamma)$ of level $\Gamma$, a congruence subgroup of $\mathrm{PSL}_{2}(\mathbb{Z})$. These extend both the ring of classical Hecke operators and the algebra of modular forms.

Modular Hecke algebras encode two a priori unrelated structures on modular forms, namely the algebra structure given by the pointwise product on one hand, and the action of the Hecke operators on the other. To any congruence subgroup $\Gamma$ of $\mathrm{SL}_{2}(\mathbb{Z})$ corresponds a crossed product algebra $\mathcal{A}(\Gamma)$, the modular Hecke algebra of level $\Gamma$, which is a direct extension of both the ring of classical Hecke operators and of the algebra $\mathcal{M}(\Gamma)$ of $\Gamma$-modular forms.

These algebras can be obtained by considering the action of $\mathrm{GL}_{2}^{+}(\mathbb{Q})$ on the algebra of modular forms on the full (adelic) modular tower, which yields the "holomorphic part" of the 
"ring of functions" of the noncommutative space of commensurability classes of 2-dimensional Q-lattices, introduced in Section 22

With $\mathcal{M}$ denoting the algebra of modular forms of arbitrary level, the elements of $\mathcal{A}(\Gamma)$ are maps with finite support

$$
F: \Gamma \backslash \mathrm{GL}^{+}(2, \mathbb{Q}) \rightarrow \mathcal{M}, \quad \Gamma \alpha \mapsto F_{\alpha} \in \mathcal{M},
$$

satisfying the covariance condition

$$
F_{\alpha \gamma}=F_{\alpha} \mid \gamma, \quad \forall \alpha \in \mathrm{GL}^{+}(2, \mathbb{Q}), \gamma \in \Gamma
$$

and their product is given by convolution.

More in detail, let $G=\mathrm{PGL}_{2}^{+}(\mathbb{Q})$ and $\Gamma \subset \mathrm{PSL}_{2}(\mathbb{Z})$ a finite index subgroup. The quotient map $\Gamma \backslash G \rightarrow \Gamma \backslash G / \Gamma$ is finite to one, and $\Gamma$ acts on $\mathbb{C}[\Gamma \backslash G]$. Let $\mathcal{H}_{k}$ be the space of holomorphic functions $f: \mathbb{H} \rightarrow \mathbb{C}$ with polynomial growth, and with the action $\left.\right|_{k}$, for $k \in 2 \mathbb{Z}$, of $\mathrm{PGL}_{2}^{+}(\mathbb{R})$ of the form

$$
\left(\left.f\right|_{k}\left(\begin{array}{ll}
a & b \\
c & d
\end{array}\right)\right)(z)=\frac{(a d-b c)^{k / 2}}{(c z+d)^{k}} f\left(\frac{a z+b}{c z+d}\right) .
$$

This determines induced actions of $G$ and $\Gamma$ on $\mathcal{H}_{k}$. The space of modular forms is obtained as $\mathcal{M}_{k}(\Gamma)=\mathcal{H}_{k}^{\Gamma}$, the invariants of this action.

One can then define

$$
\mathcal{A}_{k}(\Gamma):=\left(\mathbb{C}[\Gamma \backslash G] \otimes_{\mathbb{C}} \mathcal{H}_{k}\right)^{\Gamma},
$$

with respect to the right action of $\Gamma$,

$$
\gamma: \sum_{i}\left(\Gamma g_{i}\right) \otimes f_{i} \mapsto \sum_{i} \Gamma g_{i} \gamma \otimes\left(\left.f_{i}\right|_{k} \gamma\right)
$$

One considers the graded vector space $\mathcal{A}_{*}(\Gamma)=\oplus_{k} \mathcal{A}_{k}(\Gamma)$. The elements of $\mathcal{A}_{k}(\Gamma)$ can be thought of as finitely supported $\Gamma$-equivariant maps

$$
\phi: \Gamma \backslash G \rightarrow \mathcal{H}_{k} \quad \sum_{i}\left(\Gamma g_{i}\right) \otimes f_{i} \mapsto f_{i} .
$$

We can embed

$$
\mathcal{A}_{*}(\Gamma) \subset \hat{\mathcal{A}}_{*}(\Gamma):=\operatorname{Hom}_{\Gamma}\left(\mathbb{C}[\Gamma \backslash G], \mathcal{H}_{k}\right),
$$

where we think of $\mathcal{A}_{*}=\mathcal{H}_{*}[\Gamma \backslash G]$ as polynomials in $\Gamma \backslash G$ with $\mathcal{H}_{*}$ coefficients, and of $\hat{\mathcal{A}}_{*}=$ $\mathcal{H}_{*}[[\Gamma \backslash G]]$ as formal power series, that is, $\Gamma$-equivariant maps $\phi: \Gamma \backslash G \rightarrow \mathcal{H}_{k}$. There is on $\mathcal{A}_{*}(\Gamma)$ an associative multiplication ( $c f$. 84]), which makes $\mathcal{A}_{*}(\Gamma)$ into a noncommutative ring. This is given by a convolution product. For any $\phi \in \mathcal{A}_{k}(\Gamma)$, we have $\phi_{g}=\phi_{\gamma g}$, with $\phi_{g}=0$ off a finite subset of $\Gamma \backslash G$, and $\phi_{g} \mid \gamma=\phi_{g \gamma}$, so these terms are left $\Gamma$-invariant and right $\Gamma$-equivariant. For $\phi \in \mathcal{A}_{k}(\Gamma)$ and $\psi \in \mathcal{A}_{\ell}(\Gamma)$ we then define the convolution product as

$$
(\phi * \psi)_{g}:=\sum_{\left(g_{1}, g_{2}\right) \in G \times \times_{\Gamma} G, g_{1} g_{2}=g}\left(\phi_{g_{1}} \mid g_{2}\right) \phi_{g_{2}},
$$

The algebra $\mathcal{A}_{*}(\Gamma)$ constructed this way has two remarkable subalgebras.

- $\mathcal{A}_{0}(\Gamma)=\mathbb{C}[\Gamma \backslash G / \Gamma]$ is the algebra $\mathbb{T}$ of Hecke operators.

- $\mathcal{M}_{k}(\Gamma) \subset \mathcal{A}_{k}(\Gamma)$ also gives a subalgebra $\mathcal{M}_{*}(\Gamma) \subset \mathcal{A}_{*}(\Gamma)$. 
In particular observe that all the coefficients $\phi_{g}$ are modular forms. In fact, they satisfy $\phi_{g} \mid \gamma=\phi_{g \gamma}$, hence, for $\gamma \in \Gamma$, this gives $\phi_{g} \mid \gamma=\phi_{g}$.

Notice however that the convolution product on $\mathcal{A}_{*}$ does not agree with the Hecke action, namely the diagram

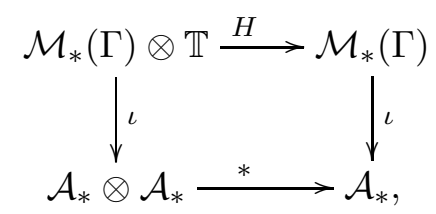

with $\iota$ the inclusion of subalgebras and $H$ the Hecke action, is not commutative, nor is the symmetric one

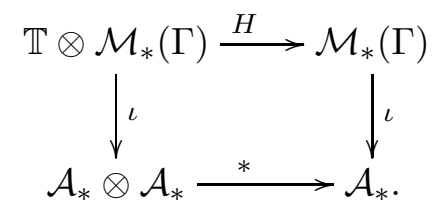

To get the correct Hecke action on modular forms from the algebra $\mathcal{A}_{*}(\Gamma)$, one needs to introduce the augmentation map

$$
\epsilon: \mathbb{C}[\Gamma \backslash G] \rightarrow \mathbb{C}
$$

extended to a map

$$
\epsilon \otimes 1: \mathbb{C}[\Gamma \backslash G] \otimes \mathcal{H}_{k} \rightarrow \mathcal{H}_{k} \quad \sum[g] \otimes \phi_{g} \mapsto \sum \phi_{g}
$$

One then obtains a commutative diagram

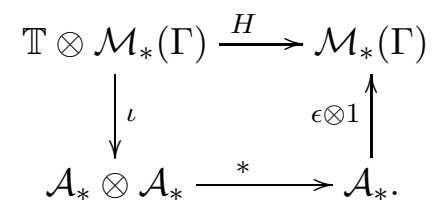

In 82 Connes and Moscovici introduced a Hopf algebra $\mathcal{H}_{1}$ associated to the transverse geometry of codimension one foliations. This is the universal enveloping algebra of a Lie algebra with basis $\left\{\mathcal{X}, \mathcal{Y}, \delta_{n} n \geq 1\right\}$ satisfying, for $n, k, \ell \geq 1$,

$$
[\mathcal{Y}, \mathcal{X}]=\mathcal{X},\left[\mathcal{Y}, \delta_{n}\right]=n \delta_{n},\left[\mathcal{X}, \delta_{n}\right]=\delta_{n+1}, \quad\left[\delta_{k}, \delta_{\ell}\right]=0
$$

with coproduct an algebra homomorphism $\Delta: \mathcal{H}_{1} \rightarrow \mathcal{H}_{1} \otimes \mathcal{H}_{1}$ satisfying

$$
\begin{aligned}
& \Delta \mathcal{Y}=\mathcal{Y} \otimes 1+1 \otimes \mathcal{Y}, \\
& \Delta \delta_{1}=\delta_{1} \otimes 1+1 \otimes \delta_{1}, \\
& \Delta \mathcal{X}=\mathcal{X} \otimes 1+1 \otimes \mathcal{X}+\delta_{1} \otimes \mathcal{Y},
\end{aligned}
$$

antipode the anti-isomorphism satisfying

$$
S(\mathcal{Y})=-\mathcal{Y}, \quad S(\mathcal{X})=-\mathcal{X}+\delta_{1} \mathcal{Y}, \quad S\left(\delta_{1}\right)=-\delta_{1},
$$

and co-unit $\epsilon(h)$ the constant term of $h \in \mathcal{H}_{1}$.

The Hopf algebra $\mathcal{H}_{1}$ acts as symmetries of the modular Hecke algebras. This is a manifestation of the general fact that, while symmetries of ordinary commutative spaces are encoded by group actions, symmetries of noncommutative spaces are given by Hopf algebras. 
By comparing the actions of the Hopf algebra $\mathcal{H}_{1}$, it is possible to derive an analogy (cf. 84]) between the modular Hecke algebras and the crossed product algebra of the action of a discrete subgroup of $\operatorname{Diff}\left(S^{1}\right)$ on polynomial functions on the frame bundle of $S^{1}$.

In fact, for $\Gamma$ a discrete subgroup of $\operatorname{Diff}\left(S^{1}\right)$, and $X$ a smooth compact 1-dimensional manifold, consider as in [84] the algebra

$$
\mathcal{A}_{\Gamma}=C_{c}^{\infty}\left(J_{+}^{1}(X)\right) \rtimes \Gamma,
$$

where $J_{+}^{1}(X)$ is the oriented 1-jet bundle. This has an action of the Hopf algebra $\mathcal{H}_{1}$ by

$$
\begin{aligned}
\mathcal{Y}\left(f U_{\phi}^{*}\right) & =y_{1} \frac{\partial f}{\partial y_{1}} U_{\phi}^{*} \\
\mathcal{X}\left(f U_{\phi}^{*}\right) & =y_{1} \frac{\partial f}{\partial y} U_{\phi}^{*} \\
\delta_{n}\left(f U_{\phi}^{*}\right) & =y_{1}^{n} \frac{d^{n}}{d y^{n}}\left(\log \frac{d \phi}{d y}\right) f U_{\phi}^{*},
\end{aligned}
$$

with coordinates $\left(y, y_{1}\right)$ on $J_{+}^{1}(X) \simeq X \times \mathbb{R}^{+}$. The trace $\tau$ defined by the volume form

$$
\tau\left(f U_{\phi}^{*}\right)= \begin{cases}\int_{J_{+}^{1}(X)} f\left(y, y_{1}\right) \frac{d y \wedge d y_{1}}{y_{1}^{2}} & \phi=1 \\ 0 & \phi \neq 1\end{cases}
$$

satisfies

$$
\tau(h(a))=\nu(h) \tau(a) \quad \forall h \in \mathcal{H}_{1},
$$

with $\nu \in \mathcal{H}_{1}^{*}$ satisfying

$$
\nu(\mathcal{Y})=1, \quad \nu(\mathcal{X})=0, \quad \nu\left(\delta_{n}\right)=0 .
$$

The twisted antipode $\tilde{S}=\nu * S$ satisfies $\tilde{S}^{2}=1$ and

$$
\tilde{S}(\mathcal{Y})=-\mathcal{Y}+1, \quad \tilde{S}(\mathcal{X})=-\mathcal{X}+\delta_{1} \mathcal{Y}, \quad \tilde{S}\left(\delta_{1}\right)=-\delta_{1} .
$$

The Hopf cyclic cohomology of a Hopf algebra is another fundamental tool in noncommutative geometry, which was developed by Connes and Moscovici in [82. They applied it to the computation of the local index formula for tranversely hypoelliptic operators on foliations. An action of a Hopf algebra on an algebra induces a characteristic map from the Hopf cyclic cohomology of the Hopf algebra to the cyclic cohomology of the algebra, hence the index computation can be done in terms of Hopf cyclic cohomology. The periodic Hopf cyclic cohomology of the Hopf algebra of transverse geometry is related to the Gelfand-Fuchs cohomology of the Lie algebra of formal vector fields 83 .

In the case of the Hopf algebra $\mathcal{H}_{1}$, there are three basic cyclic cocycles, which in the original context of transverse geometry correspond, respectively, to the Schwarzian derivative, the Godbillon-Vey class, and the transverse fundamental class.

In particular, the Hopf cyclic cocycle associated to the Schwarzian derivative is of the form

$$
\delta_{2}^{\prime}:=\delta_{2}-\frac{1}{2} \delta_{1}^{2}
$$

with

$$
\delta_{2}^{\prime}\left(f U_{\phi}^{*}\right)=y_{1}^{2}\{\phi(y) ; y\} f U_{\phi}^{*}
$$




$$
\{F ; x\}:=\frac{d^{2}}{d x^{2}}\left(\log \frac{d F}{d x}\right)-\frac{1}{2}\left(\frac{d}{d x}\left(\log \frac{d F}{d x}\right)\right)^{2} .
$$

The action of the Hopf algebra $\mathcal{H}_{1}$ on the modular Hecke algebra described in 84 involves the natural derivation on the algebra of modular forms initially introduced by Ramanujan, which corrects the ordinary differentiation by a logarithmic derivative of the Dedekind $\eta$ function,

$$
\mathcal{X}:=\frac{1}{2 \pi i} \frac{d}{d z}-\frac{1}{2 \pi i} \frac{d}{d z}\left(\log \eta^{4}\right) \mathcal{Y}, \quad \mathcal{Y}(f)=\frac{k}{2} f, \quad \forall f \in \mathcal{M}_{k} .
$$

The element $\mathcal{Y}$ is the grading operator that multiplies by $k / 2$ forms of weight $k$, viewed as sections of the $(k / 2)$ th power of the line bundle of 1 -forms. The element $\delta_{1}$ acts as multiplication by a form-valued cocycle on $\mathrm{GL}_{2}^{+}(\mathbb{Q})$, which measures the lack of invariance of the section $\eta^{4} d z$. More precisely, one has the following action of $\mathcal{H}_{1}$ (Connes-Moscovici [84):

Theorem 23.1. There is an action of the Hopf algebra $\mathcal{H}_{1}$ on the modular Hecke algebra $\mathcal{A}(\Gamma)$ of level $\Gamma$, induced by an action on $\mathcal{A}_{G^{+}(\mathbb{Q})}:=\mathcal{M} \rtimes G^{+}(\mathbb{Q})$, for $\mathcal{M}=\lim _{N \rightarrow \infty} \mathcal{M}(\Gamma(N))$, of the form

$$
\begin{aligned}
\mathcal{Y}\left(f U_{\gamma}^{*}\right) & =\mathcal{Y}(f) U_{\gamma}^{*} \\
\mathcal{X}\left(f U_{\gamma}^{*}\right) & =\mathcal{X}(f) U_{\gamma}^{*} \\
\delta_{n}\left(f U_{\gamma}^{*}\right) & =\frac{d^{n}}{d Z^{n}}\left(\log \frac{d\left(\left.Z\right|_{0} \gamma\right)}{d Z}\right)(d Z)^{n} f U_{\gamma}^{*},
\end{aligned}
$$

with $\mathcal{X}(f)$ and $\mathcal{Y}(f)$ as in (23.15), and

$$
Z(z)=\int_{i \infty}^{z} \eta^{4} d z
$$

The cocycle (23.12) associated to the Schwarzian derivative is represented by an inner derivation of $\mathcal{A}_{G^{+}(\mathbb{Q})}$,

$$
\delta_{2}^{\prime}(a)=\left[a, \omega_{4}\right]
$$

where $\omega_{4}$ is the weight four modular form

$$
\omega_{4}=-\frac{E_{4}}{72}, \quad \text { with } \quad E_{4}(q)=1+240 \sum_{n=1}^{\infty} n^{3} \frac{q^{n}}{1-q^{n}}, \quad q=e^{2 \pi i z},
$$

which is expressed as a Schwarzian derivative

$$
\omega_{4}=(2 \pi i)^{-2}\{Z ; z\} .
$$

This result is used in 84 to investigate perturbations of the Hopf algebra action. The freedom one has in modifying the action by a 1-cocycle corresponds exactly to the data introduced by Zagier in [203, defining "canonical" Rankin-Cohen algebras, with the derivation $\partial$ and the element $\Phi$ in Zagier's notation corresponding, respectively, to the action of the generator $\mathcal{X}$ on modular forms and to $\omega_{4}=2 \Phi$.

The cocycle associated to the Godbillon-Vey class is described in terms of a 1-cocycle on $\mathrm{GL}_{2}^{+}(\mathbb{Q})$ with values in Eisenstein series of weight two, which measures the lack of $\mathrm{GL}_{2}^{+}(\mathbb{Q})$ invariance of the connection associated to the generator $\mathcal{X}$. The authors derive from this an 
arithmetic presentation of the rational Euler class in $H^{2}\left(\mathrm{SL}_{2}(\mathbb{Q}), \mathbb{Q}\right)$ in terms of generalized Dedekind sums.

The cocycle associated to the transverse fundamental class, on the other hand, gives rise to a natural extension of the first Rankin-Cohen bracket 203 from modular forms to the modular Hecke algebras.

Rankin-Cohen algebras can be treated in different perspectives: Zagier introduced them and studied them with a direct algebraic approach ( $c f$. 203]). There appears to be an interesting and deep connection to vertex operator algebras, which manifests itself in a form of duality between these two types of algebras.

The Rankin-Cohen brackets are a family of brackets $[f, g]_{n}^{(k, \ell)}$ for $n \geq 0$, defined for $f, g \in R$, where $R$ is a graded ring with a derivation $D$. For $R=\oplus_{k \geq 0} R_{k}, D: R_{k} \rightarrow R_{k+2}, f \in R_{k}$ $g \in R_{\ell}$, the brackets [, $]_{n}: R_{k} \otimes R_{\ell} \rightarrow R_{k+\ell+2}$ are given by

$$
[f, g]_{n}^{(k, \ell)}=\sum_{r+s=n}(-1)^{r}\left(\begin{array}{c}
n+k-1 \\
s
\end{array}\right)\left(\begin{array}{c}
n+\ell-1 \\
r
\end{array}\right) D^{r} f D^{s} g .
$$

These Rankin-Cohen brackets induced by $\left(R_{*}, D\right) \Rightarrow\left(R_{*},[,]_{*}\right)$ give rise to a standard RankinCohen algebra, in Zagier's terminology ( $c f$. 203]). There is an isomorphism of categories between graded rings with a derivation and standard Rankin-Cohen algebras.

In the case of Lie algebras, one can first define a standard Lie algebra as the Lie algebra associated to an associative algebra $(A, *) \Rightarrow(A,[]$,$) by setting [X, Y]=X * Y-Y * X$ and then define an abstract Lie algebra as a structure $(A,[]$,$) that satisfies all the algebraic$ identities satisfied by a standard Lie algebras, though it is not necessarily induced by an associative algebra. It is then a theorem that the antisymmetry of the bracket and the Jacobi identity are sufficient to determine all the other algebraic identities, hence one can take these as a definition of an abstract Lie algebra.

Just as in the case of Lie algebras, we can define a Rankin-Cohen algebra $\left(R_{*},[,]_{*}\right)$ as a graded ring $R_{*}$ with a family of degree $2 n$ brackets [, $]_{n}$ satisfying all the algebraic identities of the standard Rankin-Cohen algebra. However, in this case there is no simple set of axioms that implies all the algebraic identities.

The motivation for this structure lies in the fact that there is a very important example of a Rankin-Cohen algebra which is in fact non-standard. The example is provided by modular forms ( $c f$. 203]).

If $f \in \mathcal{M}_{k}$ is a modular form satisfying

$$
f\left(\frac{a z+b}{c z+d}\right)=(c z+d)^{k} f(z)
$$

then it derivative is no longer a modular form, due to the presence of the second term in

$$
f^{\prime}\left(\frac{a z+b}{c z+d}\right)=(c z+d)^{k+2} f(z)+k c(c z+d)^{k+1} f(z) .
$$

On the other hand, if we have $f \in \mathcal{M}_{k}$ and $g \in \mathcal{M}_{\ell}$, the bracket

$$
[f, g](z):=\ell f^{\prime}(z) g(z)-k f(z) g^{\prime}(z)
$$


is a modular form in $\mathcal{M}_{k+\ell+2}$. Similarly, we can define an $n$-th bracket $[,]_{n}: \mathcal{M}_{k} \otimes \mathcal{M}_{\ell} \rightarrow$ $\mathcal{M}_{k+\ell+2}$. Here's the first few brackets:

$$
\begin{aligned}
& {[f, g]_{0}=f g} \\
& {[f, g]_{1}=k f g^{\prime}-\ell f^{\prime} g} \\
& {[f, g]_{2}=\left(\begin{array}{c}
k+1 \\
2
\end{array}\right) f g^{\prime \prime}-(k+1)(\ell+1) f^{\prime} g^{\prime}+\left(\begin{array}{c}
\ell+1 \\
2
\end{array}\right) f^{\prime \prime} g .}
\end{aligned}
$$

Notice that for the graded ring of modular forms we have $\mathcal{M}_{*}(\Gamma) \subset \mathcal{H}$, where $\mathcal{H}$ is the vector space $\mathcal{H}=\operatorname{Hol}(\mathbb{H})_{\text {polyn }}$ of holomorphic functions on the upper half plane $\mathbb{H}$ with polynomial growth. This is closed under differentiation and $(\mathcal{H}, D)$ induces a standard Rankin-Cohen algebra $\left(\mathcal{H},[,]_{*}\right)$. The inclusion $\left(\mathcal{M}_{*},[,]_{*}\right) \subset\left(\mathcal{H},[,]_{*}\right)$ is not closed under differentiation but it is closed under the brackets.

A way of constructing non-standard Rankin-Cohen algebras is provided by Zagier's canonical construction ( $c f$. 203]). One considers here the data $\left(R_{*}, D, \Phi\right)$, where $R_{*}$ is a graded ring with a derivation $D$ and with a choice of an element $\Phi \in R_{4}$, the curvature. One then defines the brackets by the formula

$$
[f, g]_{n}^{(k, \ell)}=\sum_{r+s=n}(-1)^{r}\left(\begin{array}{c}
n+k-1 \\
s
\end{array}\right)\left(\begin{array}{c}
n+\ell-1 \\
r
\end{array}\right) f_{r} g_{s}
$$

where $f_{0}=f$ and

$$
f_{r+1}=D f_{r}+r(r+1) \Phi f_{r-1} .
$$

The structure $\left(R_{*},[,]_{*}\right)$ obtained this way is a Rankin-Cohen algebra (see [203]).

There is a gauge action on the curvature $\Phi$, namely, for any $\varphi \in R_{2}$ the transformation $D \mapsto D^{\prime}$ and $\Phi \mapsto \Phi^{\prime}$ with

for $f \in \mathcal{M}_{k}$ and

$$
D^{\prime}(f)=D(f)+k \varphi f
$$

$$
\Phi^{\prime}=\Phi+\varphi^{2}-D(\varphi)
$$

give rise to the same Rankin-Cohen algebra. Thus, all the cases where the curvature $\Phi$ can be gauged away to zero correspond to the standard case.

The modular form $\omega_{4}$ of (23.20) provides the curvature element $\omega_{4}=2 \Phi$, and the gauge equivalence condition (23.24) can be rephrased in terms of Hopf algebras as the freedom to change the $\mathcal{H}_{1}$ action by a cocycle. In particular ( $c f$. [84]), for the specified action, the resulting Rankin-Cohen structure is canonical but not standard, in Zagier's terminology.

The 1-form $d Z=\eta^{4} d z$ is, up to scalars, the only holomorphic differential on the elliptic curve $E=X_{\Gamma(6)} \cong X_{\Gamma_{0}(36)}$ of equation $y^{2}=x^{3}+1$, so that $d Z=\frac{d x}{y}$ in Weierstrass coordinates.

The Rankin-Cohen brackets on modular forms can be extended to brackets $R C_{n}$ on the modular Hecke algebra, defined in terms of the action of the Hopf algebra $\mathcal{H}_{1}$ of transverse geometry. In fact, more generally, it is shown in [85] that it is possible to define such RankinCohen brackets on any associative algebra $\mathcal{A}$ endowed with an action of the Hopf algebra $\mathcal{H}_{1}$ for which there exists an element $\Omega \in \mathcal{A}$ such that

$$
\delta_{2}^{\prime}(a)=[\Omega, a], \quad \forall a \in \mathcal{A},
$$


and with $\delta_{2}^{\prime}$ as in (23.12), and

$$
\delta_{n}(\Omega)=0, \quad \forall n \geq 1 \text {. }
$$

Under these hypotheses, the following result holds (Connes-Moscovici [85]):

Theorem 23.2. Suppose given an associative algebra $\mathcal{A}$ with an action of the Hopf algebra $\mathcal{H}_{1}$ satisfying the conditions (23.25) and (23.26).

(1) There exists Rankin-Cohen brackets $R C_{n}$ of the form

$$
R C_{n}(a, b)=\sum_{k=0}^{n} \frac{A_{k}}{k !}(2 \mathcal{Y}+k)_{n-k}(a) \frac{B_{n-k}}{(n-k) !}(2 \mathcal{Y}+n-k)_{k}(b),
$$

with $(\alpha)_{r}=\alpha(\alpha+1) \cdots(\alpha+r-1)$ and the coefficients $A_{-1}=0, A_{0}=1, B_{0}=1$, $B_{1}=\mathcal{X}$,

$$
\begin{gathered}
A_{n+1}=S(\mathcal{X}) A_{n}-n \Omega^{0}\left(\mathcal{Y}-\frac{n-1}{2}\right) A_{n-1}, \\
B_{n+1}=\mathcal{X} B_{n}-n \Omega\left(\mathcal{Y}-\frac{n-1}{2}\right) B_{n-1},
\end{gathered}
$$

and $\Omega^{0}$ the right multiplication by $\Omega$.

(2) When applied to the modular Hecke algebra $\mathcal{A}(\Gamma)$, with $\Omega=\omega_{4}=2 \Phi$, the above construction yields brackets (23.27) that are completely determined by their restriction to modular forms where they agree with the Rankin-Cohen brackets (23.22).

(3) The brackets (23.27) determine associative deformations

$$
a * b=\sum_{n} \hbar^{n} R C_{n}(a, b) .
$$

The first of the steps described in Section 2 namely resolving the diagonal in $\mathcal{A}(\Gamma)$, is not yet done for the modular Hecke algebras and should shed light on the important number theoretic problem of the interrelation of the Hecke operators with the algebra structure given by the pointwise product.

The algebra $\mathcal{A}(\Gamma)$ is deeply related to the algebra of the space of two dimensional $\mathbb{Q}$-lattices of Section 22

\section{Noncommutative moduli spaces, Shimura varieties}

It appears from the study of some significant cases that an important source of interesting noncommutative spaces is provided by the "boundary" of classical (algebro-geometric) moduli spaces, when one takes into account the possible presence of degenerations of classical algebraic varieties that give rise to objects no longer defined within the context of algebraic varieties, but which still make sense as noncommutative spaces.

An example of algebro-geometric moduli spaces which is sufficiently simple to describe but which at the same time exhibits a very rich structure is that of the modular curves. The geometry of modular curves has already appeared behind our discussion of the 2-dimensional $\mathbb{Q}$-lattices and of the modular Hecke algebras, through an associated class of functions: the 
modular functions that appeared in our discussion of the arithmetic algebra for the quantum statistical mechanical system of 2-dimensional $\mathbb{Q}$-lattices and the modular forms in the modular Hecke algebras.

The modular curves, quotients of the hyperbolic plane $\mathbb{H}$ by the action of a subgroup $\Gamma$ of finite index of $\mathrm{SL}_{2}(\mathbb{Z})$, are complex algebraic curves, which admit an arithmetic structure, as they are defined over cyclotomic number fields $\mathbb{Q}\left(\zeta_{N}\right)$. They are also naturally moduli spaces. The object they parameterize are elliptic curves (with some level structure). The modular curves have an algebro-geometric compactification obtained by adding finitely many cusp points, given by the points in $\mathbb{P}^{1}(\mathbb{Q}) / \Gamma$. These correspond to the algebro-geometric degeneration of the elliptic curve to $\mathbb{C}^{*}$. However, in addition to these degenerations, one can consider degenerations to noncommutative tori, obtained by a limit $q \rightarrow \exp (2 \pi i \theta)$ in the modulus $q=\exp (2 \pi i \tau)$ of the elliptic curve, where now $\theta$ is allowed to be also irrational. The resulting boundary $\mathbb{P}^{1}(\mathbb{R}) / \Gamma$ is a noncommutative space (in the sense of Section 4 ). It appeared in the string theory compactifications considered in 68. The arithmetic properties of the noncommutative spaces $\mathbb{P}^{1}(\mathbb{R}) / \Gamma$ were studied in [147], [150] [151].

The modular curves, for varying finite index $\Gamma \subset \mathrm{SL}_{2}(\mathbb{Z})$, form a tower of branched coverings. The projective limit of this tower sits as a connected component in the more refined adelic version of the modular tower, given by the quotient

$$
\mathrm{GL}_{2}(\mathbb{Q}) \backslash \mathrm{GL}_{2}(\mathbb{A}) / \mathbb{C}^{*}
$$

where, as usual, $\mathbb{A}=\mathbb{A}_{f} \times \mathbb{R}$ denotes the adeles of $\mathbb{Q}$, with $\mathbb{A}_{f}=\hat{\mathbb{Z}} \otimes \mathbb{Q}$ the finite adeles. The space (24.1) is also a moduli space. In fact, it belongs to an important class of algebrogeometric moduli spaces of great arithmetic significance, the Shimura varieties $\operatorname{Sh}(G, X)$, where the data $(G, X)$ are given by a reductive algebraic group $G$ and a hermitian symmetric domain $X$. The pro-variety (24.1) is the Shimura variety $S h\left(\mathrm{GL}_{2}, \mathbb{H}^{ \pm}\right)$, where $\mathbb{H}^{ \pm}=$ $\mathrm{GL}_{2}(\mathbb{R}) / \mathbb{C}^{*}$ is the union of upper and lower half plane in $\mathbb{P}^{1}(\mathbb{C})$.

We have mentioned above that the spaces $\mathbb{P}^{1}(\mathbb{R}) / \Gamma$ describe degenerations of elliptic curves to noncommutative tori. This type of degeneration corresponds to degenerating a lattice $\Lambda=\mathbb{Z}+\mathbb{Z} \tau$ to a pseudolattice $L=\mathbb{Z}+\mathbb{Z} \theta$ (see [143] for a detailed discussion of this viewpoint and its implications in noncommutative geometry and in arithmetic). In terms of the space (24.1), it corresponds to degenerating the archimedean component, namely replacing $\mathrm{GL}_{2}(\mathbb{R})$ by $M_{2}(\mathbb{R})^{\cdot}=M_{2}(\mathbb{R}) \backslash\{0\}$, nonzero $2 \times 2$-matrices. However, when one is working with the adelic description as in (24.1), one can equally consider the possibility of degenerating a lattice at the non-archimedean components. This brings back directly the notion of $\mathbb{Q}$-lattices of Section 22

In fact, it was shown in [79] that the notions of 2-dimensional $\mathbb{Q}$-lattices and commensurability can be reformulated in terms of Tate modules of elliptic curves and isogeny. In these terms, the space of $\mathbb{Q}$-lattices corresponds to non-archimedean degenerations of the Tate module, which correspond to the "bad quotient"

$$
\mathrm{GL}_{2}(\mathbb{Q}) \backslash M_{2}\left(\mathbb{A}_{f}\right) \times \mathrm{GL}_{2}(\mathbb{R}) / \mathbb{C}^{*}
$$

The combination of these two types of degenerations yields a "noncommutative compactification" of the Shimura variety $S h\left(\mathrm{GL}_{2}, \mathbb{H}^{ \pm}\right)$which is the algebra of the "bad quotient"

$$
\mathrm{GL}_{2}(\mathbb{Q}) \backslash M_{2}(\mathbb{A})^{*} / \mathbb{C}^{*}
$$


where $M_{2}(\mathbb{A})^{\prime}$ are the elements of $M_{2}(\mathbb{A})$ with nonzero archimedean component. One recovers the Shimura variety $S h\left(\mathrm{GL}_{2}, \mathbb{H}^{ \pm}\right)$as the set of classical points (extremal KMS states at zero temperature) of the quantum statistical mechanical system associated to the noncommutative space (24.2) ( $c f .74$, 79]).

More generally, Shimura varieties are moduli spaces for certain types of motives or as moduli spaces of Hodge structures ( $c f$. e.g. [158]). A Hodge structure $(W, h)$ is a pair of a finite dimensional $\mathbb{Q}$-vector space $W$ and a homomorphism $h: \mathbb{S} \rightarrow \operatorname{GL}\left(W_{\mathbb{R}}\right)$, of the real algebraic group $\mathbb{S}=\operatorname{Res}_{\mathbb{C} / \mathbb{R}} \mathbb{G}_{m}$, with $W_{\mathbb{R}}=W \otimes \mathbb{R}$. This determines a decomposition $W_{\mathbb{R}} \otimes \mathbb{C}=$ $\oplus_{p, q} W^{p, q}$ with $\overline{W^{p, q}}=W^{q, p}$ and $h(z)$ acting on $W^{p, q}$ by $z^{-p} \bar{z}^{-q}$. This gives a Hodge filtration and a weight filtration $W_{\mathbb{R}}=\oplus_{k} W_{k}$ where $W_{k}=\oplus_{p+q=m} W^{p, q}$. The Hodge structure $(W, h)$ has weight $m$ if $W_{\mathbb{R}}=W_{m}$. It is rational if the weight filtration is defined over $\mathbb{Q}$. A Hodge structure of weight $m$ is polarized if there is a morphism of Hodge structures $\psi: W \otimes W \rightarrow$ $\mathbb{Q}(-m)$, such that $(2 \pi i)^{m} \psi(\cdot, h(i) \cdot)$ is symmetric and positive definite. Here $\mathbb{Q}(m)$ is the rational Hodge structure of weight $-2 m$, with $W=(2 \pi i)^{m} \mathbb{Q}$ with the action $h(z)=(z \bar{z})^{m}$. For a rational $(W, h)$, the subspace of $W \otimes \mathbb{Q}(m)$ fixed by the $h(z)$, for all $z \in \mathbb{C}^{*}$, is the space of "Hodge cycles".

One can then view Shimura varieties $S h(G, X)$ as moduli spaces of Hodge structures in the following way. Let $(G, X)$ be a Shimura datum and $\rho: G \rightarrow \operatorname{GL}(V)$ a faithful representation. Since $G$ is reductive, there is a finite family of tensors $\tau_{i}$ such that

$$
G=\left\{g \in \mathrm{GL}(V): g \tau_{i}=\tau_{i}\right\} .
$$

A point $x \in X$ is by construction a $G(\mathbb{R})$ conjugacy class of morphisms $h_{x}: \mathbb{S} \rightarrow G$, with suitable properties.

Consider data of the form $\left((W, h),\left\{s_{i}\right\}, \phi\right)$, where $(W, h)$ is a rational Hodge structure, $\left\{s_{i}\right\}$ a finite family of Hodge cycles, and $\phi$ a $K$-level structure, for some $K \subset G\left(\mathbb{A}_{f}\right)$, namely a $K$ orbit of $\mathbb{A}_{f}$-modules isomorphisms $\phi: V\left(\mathbb{A}_{f}\right) \rightarrow W\left(\mathbb{A}_{f}\right)$, which maps $\tau_{i}$ to $s_{i}$. Isomorphisms of such data are isomorphisms $f: W \rightarrow W^{\prime}$ of rational Hodge structures, sending $s_{i} \mapsto s_{i}^{\prime}$, and such that $f \circ \phi=\phi^{\prime} k$, for some $k \in K$.

We assume that there exists an isomorphism of $\mathbb{Q}$-vector spaces $\beta: W \rightarrow V$ mapping $s_{i} \mapsto \tau_{i}$ and $h$ to $h_{x}$, for some $x \in X$.

One denotes by $\operatorname{Hodge}(G, X, K)$ the set data $\left((W, h),\left\{s_{i}\right\}, \phi\right)$. The Shimura variety

$$
S h_{K}(G, X)=G(\mathbb{Q}) \backslash X \times G\left(\mathbb{A}_{f}\right) / K
$$

is the moduli space of the isomorphism classes of data $\left((W, h),\left\{s_{i}\right\}, \phi\right)$, namely there is a map of Hodge $(G, X, K)$ to $S h_{K}(G, X)$ (seen over $\mathbb{C}$ ), that descends to a bijection on isomorphism classes $\operatorname{Hodge}(G, X, K) / \sim$.

In such cases also one can consider degenerations of these data, both at the archimedean and at the nonarchimedean components. One then considers data $\left((W, h),\left\{s_{i}\right\}, \phi, \tilde{\beta}\right)$, with a non-trivial homomorphism $\tilde{\beta}: W \rightarrow V$, which is a morphism of Hodge structures, and such that $\tilde{\beta}\left(\ell_{s_{i}}\right) \subset \ell_{\tau_{i}}$. This yields noncommutative spaces inside which the classical Shimura variety sits as the set of classical points.

Quantum statistical machanical systems associated to Shimura varieties have been recently studied by Ha and Paugam in 112. Given a faithful representation $\rho: G \rightarrow \operatorname{GL}(V)$ as above, there is an "enveloping semigroup" $M$, that is, a normal irreducible semigroup $M \subset \operatorname{End}(V)$ such that $M^{\times}=G$. Such semigroup can be used to encode the degenerations of the Hodge 
data described above. The data $(G, X, V, M)$ then determine a noncommutative space which describes the "bad quotient" $S h_{K}^{n c}(G, X)=G(\mathbb{Q}) \backslash X \times M\left(\mathbb{A}_{f}\right)$ and is a moduli space for the possibly non-invertible data $\left((W, h),\left\{s_{i}\right\}, \phi\right)$. Its "set of classical points" is the Shimura variety $S h(G, X)$. The actual construction of the algebras involves some delicate steps, especially to handle the presence of stacky singularities ( $c f .[112$ ]).

\section{The ADELES ClASS SPACE AND THE SPECTRAL REALIZATION}

In this section we describe a noncommutative space, the adele class space $X_{\mathbb{K}}$, associated to any global field $\mathbb{K}$, which leads to a spectral realization of the zeros of the Riemann zeta function for $\mathbb{K}=\mathbb{Q}$ and more generally of $L$-functions associated to Hecke characters. It also gives a geometric interpretation of the Riemann-Weil explicit formulas of number theory as a trace formula. This space is closely related for $\mathbb{K}=\mathbb{Q}$ with the space of commensurability classes of $\mathbb{Q}$-lattices described above. Rather than starting directly with its description we first put the problem of finding the geometry of the set of prime numbers in the proper perspective.

\section{The set of primes}

One of the main problems of arithmetic is to understand the distribution of the set of prime numbers

$$
\{2,3,5,7,11,13,17,19,23,29,31,37,41,43,47,53,59,61,67,71, \ldots\}
$$

as a subset of the integers. To that effect one introduces the counting function

$$
\pi(n)=\text { number of primes } p \leq n
$$

The problem is to understand the behavior of $\pi(x)$ when $x \rightarrow \infty$. One often hears that the problem comes from the lack of a "simple" formula for $\pi(x)$. This is not really true and for instance in $1898 \mathrm{H}$. Laurent [139] gave the following formula whose validity is an easy exercise in arithmetic,

$$
\pi(n)=2+\sum_{k=5}^{n} \frac{e^{2 \pi i \Gamma(k) / k}-1}{e^{-2 \pi i / k}-1},
$$

where $\Gamma(k)=(k-1)$ ! is the Euler Gamma function.

The problem with this formula is that it has no bearing on the asymptotic expansion of $\pi(x)$ when $x \rightarrow \infty$. Such an expansion was guessed by Gauss in the following form,

$$
\pi(x)=\int_{0}^{x} \frac{d u}{\log (u)}+R(x)
$$

where the integral logarithm admits the asymptotic expansion,

$$
\operatorname{Li}(x)=\int_{0}^{x} \frac{d u}{\log (u)} \sim \sum(k-1) ! \frac{x}{\log (x)^{k}}
$$

The key issue then is the size of the remainder $R(x)$.

\section{The Riemann hypothesis}

It asserts that this size is governed by

$$
R(x)=O(\sqrt{x} \log (x)) .
$$




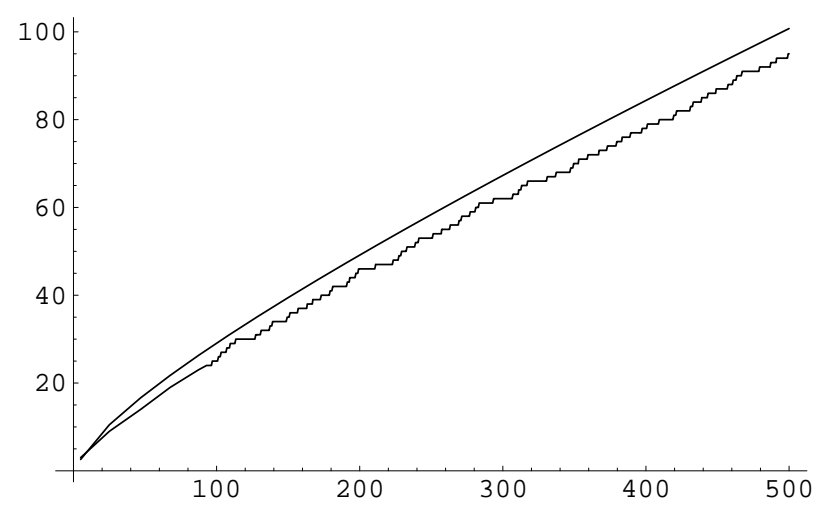

FiguRE 16. Graphs of $\pi(x)$ and $\operatorname{Li}(x)$

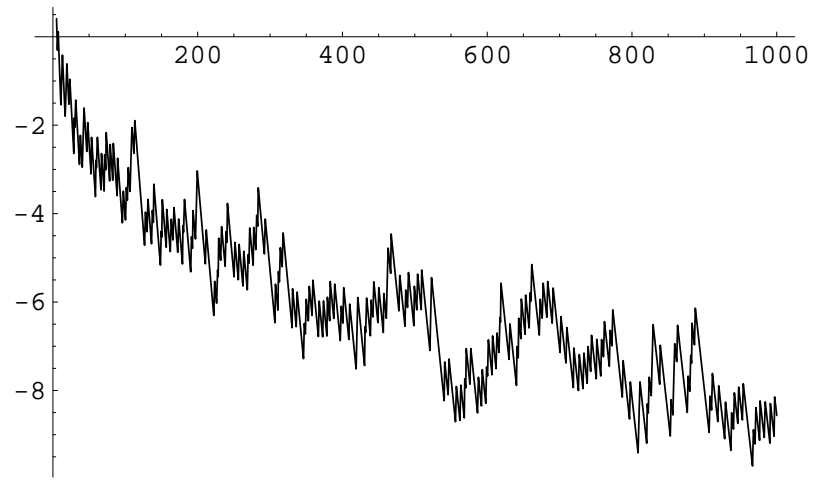

Figure 17. Graph of $\pi(x)-\operatorname{Li}(x)$

The Riemann Hypothesis is in fact a conjecture on the zeros of the zeta function

$$
\zeta(s)=\sum_{1}^{\infty} n^{-s},
$$

whose definition goes back to Euler, who showed the fundamental factorization

$$
\zeta(s)=\prod_{\mathcal{P}}\left(1-p^{-s}\right)^{-1} .
$$

It extends to a meromorphic function in the whole complex plane $\mathbb{C}$ and fulfills the functional equation

$$
\pi^{-s / 2} \Gamma(s / 2) \zeta(s)=\pi^{-(1-s) / 2} \Gamma((1-s) / 2) \zeta(1-s),
$$

so that the function

$$
\zeta_{\mathbb{Q}}(s)=\pi^{-s / 2} \Gamma(s / 2) \zeta(s)
$$

admits the symmetry $s \mapsto 1-s$. 


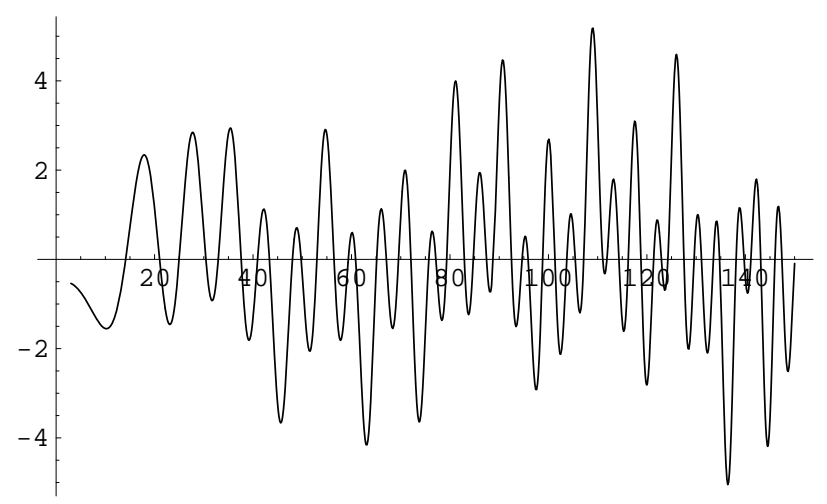

FiguRE 18. Zeros of zeta

The Riemann conjecture asserts that all zeros of $\zeta_{\mathbb{Q}}$ are on the critical line $\frac{1}{2}+i \mathbb{R}$. The reason why the location of the zeros of $\zeta_{\mathbb{Q}}$ controls the size of the remainder in (25.1) is the explicit formulas that relate primes with these zeros. Riemann proved the following first instance of an "explicit formula"

$$
\pi^{\prime}(x)=\operatorname{Li}(x)-\sum_{\rho} \operatorname{Li}\left(x^{\rho}\right)+\int_{x}^{\infty} \frac{1}{u^{2}-1} \frac{d u}{u \log u}-\log 2
$$

where the sum is over the non-trivial (i.e. complex) zeros of the zeta function, and where the function $\pi^{\prime}(x)$ is given by

$$
\pi^{\prime}(x)=\pi(x)+\frac{1}{2} \pi\left(x^{\frac{1}{2}}\right)+\frac{1}{3} \pi\left(x^{\frac{1}{3}}\right)+\cdots
$$

which gives by the Moebius inversion formula

$$
\pi(x)=\sum \mu(m) \frac{1}{m} \pi^{\prime}\left(x^{\frac{1}{m}}\right) .
$$

\section{The generalized Riemann hypothesis}

The explicit formulas of Riemann were put in more modern form by A. Weil, as

$$
\widehat{h}(0)+\widehat{h}(1)-\sum_{\rho} \widehat{h}(\rho)=\sum_{v} \int_{\mathbb{K}_{v}^{*}}^{\prime} \frac{h\left(u^{-1}\right)}{|1-u|} d^{*} u,
$$

where $\mathbb{K}$ is now an arbitrary global field, $v \in \Sigma_{\mathbb{K}}$ varies among the places of $\mathbb{K}$ and the integral is taking place over the locally compact field $\mathbb{K}_{v}$ obtained by completion of $\mathbb{K}$ at the place $v$. Also $\int^{\prime}$ is the pairing with the distribution on $\mathbb{K}_{v}$ which agrees with $\frac{d u}{|1-u|}$ for $u \neq 1$ and whose Fourier transform (relative to a selfdual choice of additive characters $\alpha_{v}$ ) vanishes at 1 . By definition a global field is a (countable) discrete cocompact subfield in a locally compact ring. This ring depends functorially on $\mathbb{K}$ and is called the ring $\mathbb{A}_{\mathbb{K}}$ of adeles of $\mathbb{K}$. The quotient

$$
C_{\mathbb{K}}=\mathrm{GL}_{1}\left(\mathbb{A}_{\mathbb{K}}\right) / \mathrm{GL}_{1}(\mathbb{K})
$$


is the locally compact group of idele classes of $\mathbb{K}$ which plays a central role in class field theory. In Weil's explicit formula the test function $h$ is in the Bruhat-Schwartz space $\mathcal{S}\left(C_{\mathbb{K}}\right)$. The multiplicative groups $\mathrm{GL}_{1}\left(\mathbb{K}_{v}\right)=\mathbb{K}_{v}^{*}$ are embedded canonically as cocompact subgroups of $C_{\mathbb{K}}$. The sum on the left hand side takes place over the zeros of $L$-functions associated to Hecke characters. The function $\widehat{h}$ is the Fourier transform of $h$. The generalized Riemann conjecture asserts that all the zeros of these $L$-functions are on the critical line $\frac{1}{2}+i \mathbb{R}$. This was proved by Weil when the global field $\mathbb{K}$ has non-zero characteristic, but remains open in the case when $\mathbb{K}$ is of characteristic zero, in which case it is a number field i.e. a finite algebraic extension of the field $\mathbb{Q}$ of rational numbers.

\section{Quantum Chaos $\rightarrow$ Riemann Flow ?}

For $E>0$ let $N(E)$ be the number of zeros of the Riemann zeta function $\zeta_{\mathbb{Q}}$ whose imaginary parts are in the open interval ]0, $E$ [. Riemann proved that the step function $N(E)$ can be written as the sum

$$
N(E)=\langle N(E)\rangle+N_{\text {osc }}(E)
$$

of a smooth approximation $\langle N(E)\rangle$ and a purely oscillatory function $N_{\text {osc }}(E)$ and gave the following explicit form

$$
\langle N(E)\rangle=\frac{E}{2 \pi}\left(\log \frac{E}{2 \pi}-1\right)+\frac{7}{8}+o(1)
$$

for the smooth approximation.

There is a striking analogy between the behavior of the step function $N(E)$ and that of the function counting the number of eigenvalues of the Hamiltonian $H$ of the quantum system obtained after quantization of a chaotic dynamical system, which is at centerstage in the theory of quantum chaos. A comparison of the asymptotic expansions of the oscillatory terms in both cases, namely

$$
N_{\mathrm{osc}}(E) \sim \frac{1}{\pi} \sum_{\gamma_{p}} \sum_{m=1}^{\infty} \frac{1}{m} \frac{1}{2 \operatorname{sh}\left(\frac{m \lambda_{p}}{2}\right)} \sin \left(m E T_{\gamma}^{\#}\right)
$$

for the quantization of a chaotic dynamical system, and

$$
N_{\text {osc }}(E) \sim \frac{-1}{\pi} \sum_{p} \sum_{m=1}^{\infty} \frac{1}{m} \frac{1}{p^{m / 2}} \sin (m E \log p)
$$

for the Riemann zeta function, gives precious indications on the hypothetical Riemann flow that would make it possible to identify the zeros of zeta as the spectrum of an Hamiltonian. For instance the periodic orbits of the flow should be labeled by the prime numbers and the corresponding periods $T_{p}$ should be given by the $\log p$. However a closer look reveals an overall minus sign that forbids any direct comparison.

\section{Spectral realization as an absorption spectrum}

The above major sign obstruction was bypassed in 64 using the following basic distinction between observed spectra in physics. When the light coming from a hot chemical element is decomposed through a prism, it gives rise to bright emission lines on a dark background, 


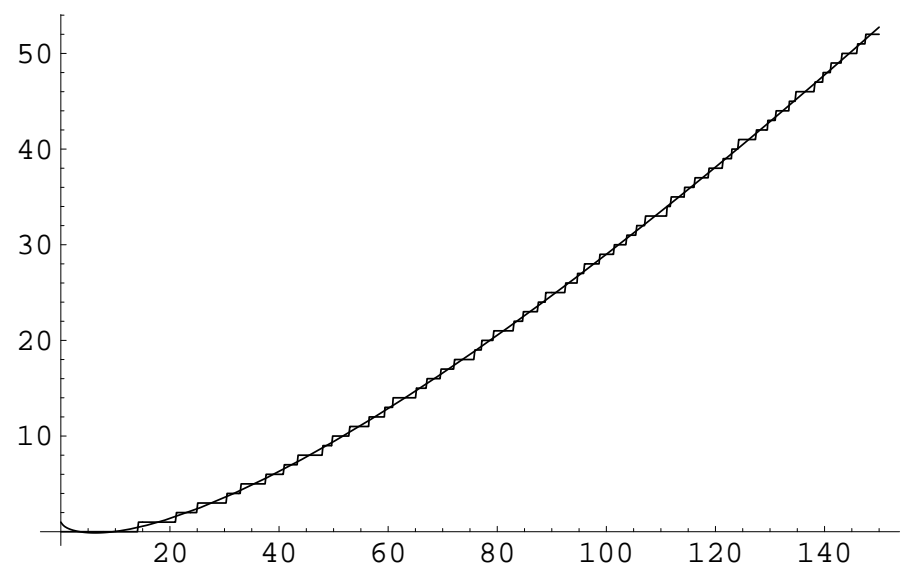

Figure 19. Counting zeros of zeta

and the corresponding frequencies are a signature of its chemical composition. When the light coming from a distant star is decomposed through a prism, it gives rise to dark lines, called absorption lines, on a white background. The spectrum of the light emitted by the sun was the first observed example of an absorption spectrum. In this case the absorption lines were discovered by Fraunhofer. The chemicals in the outer atmosphere of the star absorb the corresponding frequencies in the white light coming from the core of the star.

The simple idea then is that, because of the minus sign above, one should look for the spectral realization of the zeros of zeta not as a usual emission spectrum but as an absorption spectrum. Of course by itself this idea does not suffice to get anywhere since one needs the basic dynamical system anyway. The adele class space, namely the quotient

$$
X_{\mathbb{K}}=\mathbb{A}_{\mathbb{K}} / \mathbb{K}^{*}
$$

introduced in 64], does the job as shown there. The action of the idele class group $C_{\mathbb{K}}$ on the adele class space is simply given by multiplication. In particular the idele class group $C_{\mathbb{K}}$ acts on the suitably defined Hilbert space $L^{2}\left(X_{\mathbb{K}}\right)$ and the zeros of $L$-functions give the absorption spectrum, with non-critical zeros appearing as resonances.

Exactly as adeles, the adele class space $X_{\mathbb{K}}$ involves all the places of $\mathbb{K}$. If in order to simplify, one restricts to a finite set of places one still finds a noncommutative space but one can analyze the action of the analogue of $C_{\mathbb{K}}$ and compute its trace after performing a suitable cutoff (necessary in all cases to see the missing lines of an absorption spectrum). One gets a trace formula

$$
\operatorname{Trace}\left(R_{\Lambda} U(h)\right)=2 h(1) \log ^{\prime} \Lambda+\sum_{v \in S} \int_{\mathbb{K}_{v}^{*}}^{\prime} \frac{h\left(u^{-1}\right)}{|1-u|} d^{*} u+o(1),
$$

where the terms in the right hand side are exactly the same as in Weil's explicit formula (25.7). This is very encouraging since at least it gives geometric meaning to the complicated terms of (25.7) as the contributions of the periodic orbits to the computation of the trace. 


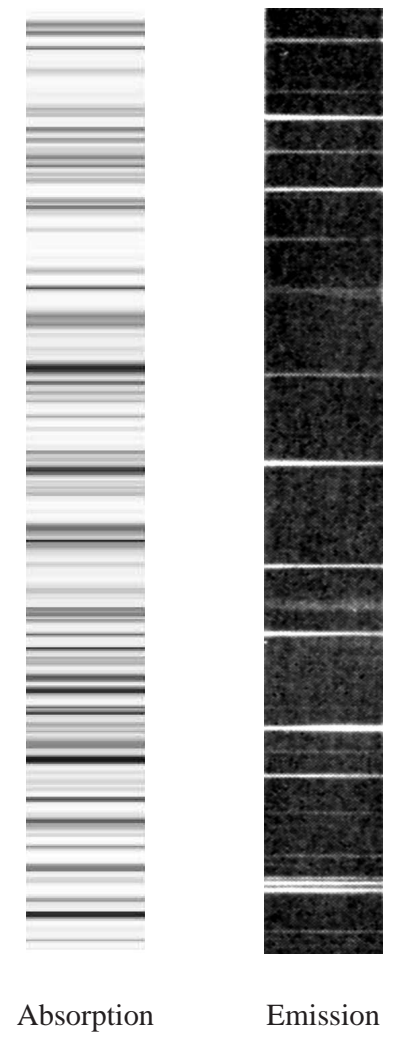

Figure 20. The two kinds of Spectra

In particular it gives a perfect interpretation of the smooth function $\langle N(E)\rangle$ approximating the counting $N(E)$ of the zeros of zeta, from counting the number of states of the one dimensional quantum system with Hamiltonian

$$
h(q, p)=2 \pi q p
$$

which is just the generator of the scaling group. Indeed the function $\frac{E}{2 \pi}\left(\log \frac{E}{2 \pi}-1\right)$ of Riemann formula (25.9) appears as the number of missing degrees of freedom in the number of quantum states for the above system, as one obtains from the simple computation of the area of the region

$$
\begin{gathered}
B_{+}=\left\{(p, q) \in[0, \Lambda]^{2} ; h(p, q) \leq E\right\} \\
\operatorname{Area}\left(B_{+}\right)=\frac{E}{2 \pi} \times 2 \log \Lambda-\frac{E}{2 \pi}\left(\log \frac{E}{2 \pi}-1\right)
\end{gathered}
$$

while the term $\frac{E}{2 \pi} \times 2 \log \Lambda$ corresponds to the number of degrees of freedom of white light. A careful computation gives not only the correction term of $\frac{7}{8}$ in (25.9) but all the remaining $o(1)$ terms. 


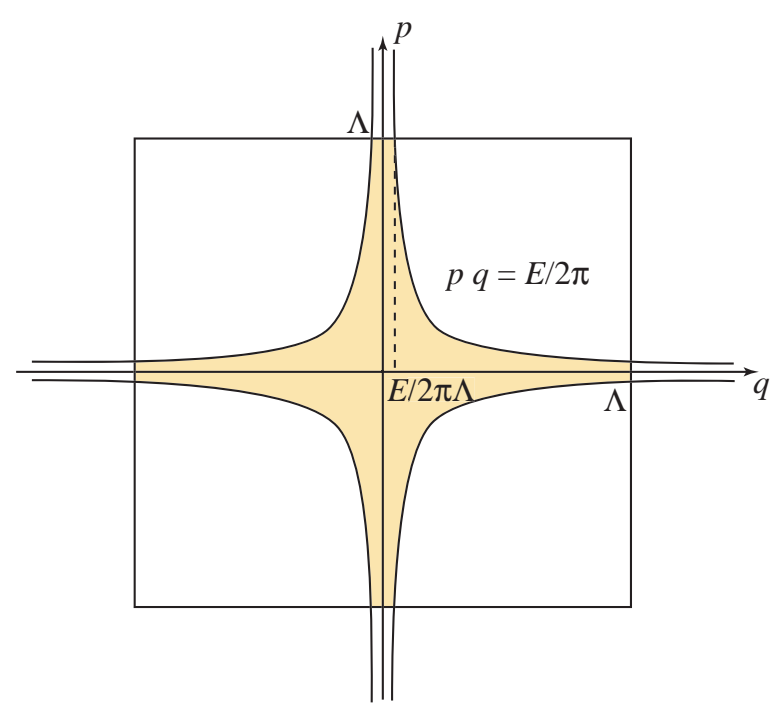

Figure 21. Counting quantum states

Finally it was shown in 64 that the generalized Riemann hypothesis is equivalent to the validity of a global trace formula, but this is only one of many equivalent reformulations of the Riemann hypothesis. ${ }^{2}$

\section{Thermodynamics of endomotives and the Tehran Program}

In many ways the great virtue of a problem like RH comes from the developments that it generates. At first sight it does not appear as having any relation with geometry, and its geometric nature gradually emerged in the twentieth century mainly because of the solution of Weil in the case of global fields of positive characteristic.

We outline a program, current joint work of Katia Consani and the two authors, to adapt Weil's proof for the case of global fields of positive characteristic to the case of number fields. ${ }^{3}$

\section{Function Fields}

Given a global field $\mathbb{K}$ of positive characteristic, there exists a finite field $\mathbb{F}_{q}$ and a smooth projective curve $C$ defined over $\mathbb{F}_{q}$ such that $\mathbb{K}$ is the field of $\mathbb{F}_{q}$-valued rational functions on $C$. The analogue (Artin, Hasse, Schmidt) of the zeta function is

$$
\zeta_{\mathbb{K}}(s)=\prod_{v \in \Sigma_{K}}\left(1-q^{-f(v) s}\right)^{-1}
$$

where $\Sigma_{\mathbb{K}}$ is the set of places of $\mathbb{K}$ and $f(v)$ is the degree (see below) of the place $v \in \Sigma_{\mathbb{K}}$.

\footnotetext{
${ }^{2}$ There is a running joke, inspired by the European myth of Faust, about a mathematician trying to bargain with the devil for a proof of the Riemann hypothesis ...

${ }^{3}$ This program was first announced in a lecture at IPM Tehran in September 2005, hence we refer to it as "the Tehran program".
} 
The functional equation takes the form

$$
q^{(g-1)(1-s)} \zeta_{\mathbb{K}}(1-s)=q^{(g-1) s} \zeta_{\mathbb{K}}(s)
$$

where $g$ is the genus of $C$.

The analogue of the Riemann conjecture for such global fields was proved by Weil (1942) who developed algebraic geometry in that context. Weil's proof rests on two steps.

- (A) Explicit Formula

- (B) Positivity

Both are based on the geometry of the action of the Frobenius on the set $C\left(\overline{\mathbb{F}}_{q}\right)$ of points of $C$ over an algebraic closure $\overline{\mathbb{F}}_{q}$ of $\mathbb{F}_{q}$. This set $C\left(\overline{\mathbb{F}}_{q}\right)$ maps canonically to the set $\Sigma_{\mathbb{K}}$ of places of $\mathbb{K}$ and the degree of a place $v \in \Sigma_{\mathbb{K}}$ is the number of points in the orbit of the Frobenius acting on the fiber of the projection

$$
C\left(\overline{\mathbb{F}}_{q}\right) \rightarrow \Sigma_{\mathbb{K}}
$$

The analogue

$$
\#\left\{C\left(\mathbb{F}_{q^{j}}\right)\right\}=\sum(-1)^{k} \operatorname{Tr}\left(\operatorname{Fr}^{* j} \mid H_{\mathrm{et}}^{k}\left(\bar{C}, \mathbb{Q}_{\ell}\right)\right)
$$

of the Lefschetz fixed point formula makes it possible to compute the number $\#\left\{C\left(\mathbb{F}_{q^{j}}\right)\right\}$ of points with coordinates in the finite extension $\mathbb{F}_{q^{j}}$ from the action of $\mathrm{Fr}^{*}$ in the etale cohomology group $H_{\mathrm{et}}^{1}\left(\bar{C}, \mathbb{Q}_{\ell}\right)$, which does not depend upon the choice of the $\ell$-adic coefficients $\mathbb{Q}_{\ell}$.

This shows that the zeta function is a rational fraction

$$
\zeta_{\mathbb{K}}(s)=\frac{P\left(q^{-s}\right)}{\left(1-q^{-s}\right)\left(1-q^{1-s}\right)}
$$

where the polynomial $P$ is the characteristic polynomial of the action of $\mathrm{Fr}^{*}$ in $H^{1}$.

The analogue of the Riemann conjecture for global fields of characteristic $p$ means that its eigenvalues i.e. the complex numbers $\lambda_{j}$ of the factorization

$$
P(T)=\prod\left(1-\lambda_{j} T\right)
$$

are of modulus $\left|\lambda_{j}\right|=q^{1 / 2}$.

The main ingredient in the proof of Weil is the notion of correspondence, given by divisors in $C \times C$. They can be viewed as multivalued maps,

$$
Z: C \rightarrow C, \quad P \mapsto Z(P) .
$$

Two correspondences are equivalent if they differ by a principal divisor,

$$
U \sim V \Leftrightarrow U-V=(f)
$$

The composition of correspondences is

$$
Z=Z_{1} \star Z_{2}, \quad Z_{1} \star Z_{2}(P)=Z_{1}\left(Z_{2}(P)\right)
$$

and the adjoint is given using the transposition $\sigma(x, y)=(y, x)$ by

$$
Z^{\prime}=\sigma(Z) \text {. }
$$

The degree $d(Z)$ of a correspondence is defined, independently of a generic point $P \in C$ by,

$$
d(Z)=Z \bullet(P \times C)
$$


where $\bullet$ is the intersection number. One has a similar definition of the codegree

$$
d^{\prime}(Z)=Z \bullet(C \times P)
$$

Weil defines the Trace of a correspondence as follows

$$
\operatorname{Trace}(Z)=d(Z)+d^{\prime}(Z)-Z \bullet \Delta
$$

where $\Delta$ is the identity correspondence. The main step in Weil's proof is

Theorem 26.1. (Weil) The following positivity holds: $\operatorname{Trace}\left(Z \star Z^{\prime}\right)>0$ unless $Z$ is a trivial class.

Clearly if one wants to have any chance at imitating the steps of Weil's proof of RH for the case of number fields one needs to have an analogue of the points of $C\left(\overline{\mathbb{F}}_{q}\right)$ and the action of the Frobenius, of the etale cohomology and of the unramified extensions $\mathbb{K} \otimes_{\mathbb{F}_{q}} \mathbb{F}_{q^{n}}$ of $\mathbb{K}$.

\section{Endomotives and Galois action}

The adele class space $X_{\mathbb{K}}$ of a global field admits a natural action of the idele class group $C_{\mathbb{K}}$ and as such is on the adelic side of the class field theory isomorphism. In order to obtain a description of this space which is closer to geometry one needs to pass to the Galois side of class field theory. In the case $\mathbb{K}=\mathbb{Q}$, it is possible to present the adele class space in a fairly simple manner not involving adeles thanks to its intimate relation with the space of 1-dimensional $\mathbb{Q}$-lattices of section [22. The direct interpretation of the action of the Galois group of $\overline{\mathbb{Q}} / \mathbb{Q}$ on the values of fabulous states for the BC-system (section 22) then suggests that one should be able to construct directly the space $X_{\mathbb{Q}}$ with a canonical action of the Galois group of $\overline{\mathbb{Q}} / \mathbb{Q}$.

This was done in 66 thanks to an extension of the notion of Artin motives, called endomotives. Following Grothendieck, one can reformulate Galois theory over a field $\mathbb{K}$ as the equivalence of the category of reduced commutative finite dimensional algebras over $\mathbb{K}$ with the category of continuous actions of the Galois group $G$ of $\mathbb{K} / \mathbb{K}$ on finite sets. By construction the algebra of the BC-system is a crossed product of a commutative algebra $A$ by a semi-group. When working over $\mathbb{K}=\mathbb{Q}$ which is essential in the definition of fabulous states, the algebra $A$ is simply the group $\operatorname{ring} \mathbb{Q}[\mathbb{Q} / \mathbb{Z}]$ of the torsion group $\mathbb{Q} / \mathbb{Z}$. Thus

$$
A=\lim _{\longrightarrow} A_{n}, \quad A_{n}=\mathbb{Q}[\mathbb{Z} / n \mathbb{Z}]
$$

and we are dealing with a projective limit of Artin motives. The key point then is to keep track of the corresponding action of the Galois group $G$ of $\mathbb{K} / \mathbb{K}, \mathbb{K}=\mathbb{Q}$. The Galois-Grothendieck correspondence associates to a reduced commutative finite dimensional algebra $B$ over $\mathbb{K}$ the set of characters of $B$ with values in $\overline{\mathbb{K}}$ together with the natural action of $G$. This action is non-trivial for the algebras $A_{n}=\mathbb{Q}[\mathbb{Z} / n \mathbb{Z}]$ where it corresponds to the cyclotomic theory. One can then recover the Bost-Connes system with its natural Galois symmetry in a conceptual manner which extends to the general context of semigroup actions on projective systems of Artin motives. These typically arise from self-maps of algebraic varieties. Given a pointed algebraic variety $\left(Y, y_{0}\right)$ over a field $\mathbb{K}$ and a countable unital abelian semigroup $S$ of finite endomorphisms of $\left(Y, y_{0}\right)$, unramified over $y_{0} \in Y$ one constructs a projective system of Artin motives $X_{s}$ over $\mathbb{K}$ from these data as follows. For $s \in S$, one sets

$$
X_{s}=\left\{y \in Y: s(y)=y_{0}\right\} .
$$


For a pair $s, s^{\prime} \in S$, with $s^{\prime}=s r$, the map $\xi_{s^{\prime}, s}: X_{s r} \rightarrow X_{s}$ is given by

$$
X_{s r} \ni y \mapsto r(y) \in X_{s} .
$$

This defines a projective system indexed by the semigroup $S$ itself with partial order given by divisibility. We let $X=\lim _{s} X_{s}$.

Since $s\left(y_{0}\right)=y_{0}$, the base point $y_{0}$ defines a component $Z_{s}$ of $X_{s}$ for all $s \in S$. Let $\xi_{s^{\prime}, s}^{-1}\left(Z_{s}\right)$ be the inverse image of $Z_{s}$ in $X_{s^{\prime}}$. It is a union of components of $X_{s^{\prime}}$. This defines a projection $e_{s}$ onto an open and closed subset $X^{e_{s}}$ of the projective limit $X$. One then shows (66]) that the semigroup $S$ acts on the projective limit $X$ by partial isomorphisms $\rho_{s}: X \rightarrow X^{e_{s}}$ defined by the property that

$$
\xi_{s u}\left(\rho_{s}(x)\right)=\xi_{u}(x), \forall u \in S, \forall x \in X .
$$

The BC-system is obtained from the pointed algebraic variety $\left(\mathbb{G}_{m}(\mathbb{Q}), 1\right)$ where the affine group scheme $\mathbb{G}_{m}$ is the multiplicative group. The semigroup $S$ is the semigroup of non-zero endomorphisms of $\mathbb{G}_{m}$. These correspond to maps of the form $u \mapsto u^{n}$ for some non-zero $n \in \mathbb{Z}$, and one restricts to $n \in \mathbb{N}^{*}$.

In this class of examples one has an "equidistribution" property, by which the uniform normalized counting measures $\mu_{s}$ on $X_{s}$ are compatible with the projective system and define a probability measure on the limit $X$. Namely, one has

$$
\xi_{s^{\prime}, s} \mu_{s}=\mu_{s^{\prime}}, \quad \forall s, s^{\prime} \in S .
$$

This follows from the fact that the number of preimages of a point under $s \in S$ is equal to $\operatorname{deg} s$. This provides exactly the data which makes it possible to perform the thermodynamical analysis of such endomotives. This gives a rather unexplored new territory since even the simplest examples beyond the BC-system remain to be investigated. For instance let $Y$ be an elliptic curve defined over $\mathbb{K}$. Let $S$ be the semigroup of non-zero endomorphisms of $Y$. This gives rise to an example in the general class described above. When the elliptic curve has complex multiplication, this gives rise to a system which, in the case of a maximal order, agrees with the one constructed in 78. In the case without complex multiplication, this provides an example of a system where the Galois action does not factor through an abelian quotient.

\section{Frobenius as dual of the time evolution}

The Frobenius is such a universal symmetry in characteristic $p$, owing to the linearity of the map $x \mapsto x^{p}$ that it is very hard to find an analogue of such a far reaching concept in characteristic zero. As we now explain, the classification of type III factors provides the basic ingredient which when combined with cyclic cohomology makes it possible to analyze the thermodynamics of a noncommutative space and get an analogue of the action of the Frobenius on etale cohomology.

The key ingredient is that noncommutativity generates a time evolution at the "measure theory" level. While it had been long known by operator algebraists that the theory of vonNeumann algebras represents a far reaching extension of measure theory, the main surprise which occurred at the beginning of the seventies in (Connes [51]) following Tomita's theory is that such an algebra $M$ inherits from its noncommutativity a god-given time evolution:

$$
\delta: \mathbb{R} \longrightarrow \text { Out } M=\text { Aut } M / \operatorname{Inn} M
$$


where Out $M=$ Aut $M / \operatorname{Inn} M$ is the quotient of the group of automorphisms of $M$ by the normal subgroup of inner automorphisms. This led in [51] to the reduction from type III to type II and their automorphisms and eventually to the classification of injective factors. They are classified by their module,

$$
\operatorname{Mod}(M) \underset{\sim}{\subset} \mathbb{R}_{+}^{*}
$$

which is a virtual closed subgroup of $\mathbb{R}_{+}^{*}$ in the sense of G. Mackey, i.e. an ergodic action of $\mathbb{R}_{+}^{*}$, called the flow of weights [90. This invariant was first defined and used in [51, to show in particular the existence of hyperfinite factors which are not isomorphic to Araki-Woods factors.

The "measure theory" level i.e. the set-up of von-Neumann algebras does not suffice to obtain the relevant cohomology theory and one needs to be given a weakly dense subalgebra $\mathcal{A} \subset M$ playing the role of smooth functions on the noncommutative space. This algebra will play a key role when cyclic cohomology is used at a later stage. At first one only uses its norm closure $A=\overline{\mathcal{A}}$ in $M$ and assumes that it is globally invariant under the modular automorphism group $\sigma_{t}^{\varphi}$ of a faithful normal state $\varphi$ on $M$. One can then proceed with the thermodynamics of the $C^{*}$ dynamical system $\left(A, \sigma_{t}\right)$. By a very simple procedure assuming that KMS states at low temperature are of type I, one obtains a "cooling morphism" $\pi$ which is a morphism of algebras from the crossed product $\hat{\mathcal{A}}=\mathcal{A} \rtimes_{\sigma} \mathbb{R}$ to a type I algebra of compact operator valued functions on a canonical $\mathbb{R}_{+}^{*}$-principal bundle $\tilde{\Omega}_{\beta}$ over the space $\Omega_{\beta}$ of type I extremal $\mathrm{KMS}_{\beta}$ states fulfilling a suitable regularity condition ( $c f$. [66]). Any $\varepsilon \in \Omega_{\beta}$ gives an irreducible representation $\pi_{\varepsilon}$ of $\mathcal{A}$ and the choice of its essentially unique extension to $\hat{\mathcal{A}}$ determines the fiber of the $\mathbb{R}_{+}^{*}$-principal bundle $\tilde{\Omega}_{\beta}$. The cooling morphism is then given by,

$$
\pi_{\varepsilon, H}\left(\int x(t) U_{t} d t\right)=\int \pi_{\varepsilon}(x(t)) e^{i t H} d t .
$$

This morphism is equivariant for the dual action $\theta_{\lambda} \in \operatorname{Aut}(\hat{\mathcal{A}})$ of $\mathbb{R}_{+}^{*}$,

$$
\theta_{\lambda}\left(\int x(t) U_{t} d t\right)=\int \lambda^{i t} x(t) U_{t} d t
$$

The key point is that the range of the morphism $\pi$ is contained in an algebra of functions on $\tilde{\Omega}_{\beta}$ with values in trace class operators. In other words modulo a Morita equivalence one lands in the commutative world provided one lowers the temperature.

The interesting space is obtained by "distillation" and is simply given by the cokernel of the cooling morphism $\pi$ but this does not make sense in the category of algebras and algebra homomorphisms since the latter is not even an additive category. This is where cyclic cohomology enters the scene : the category of cyclic modules is an abelian category with a natural functor from the category of algebras and algebra homomorphisms.

Cyclic modules are modules of the cyclic category $\Lambda$ which is a small category, obtained by enriching with cyclic morphisms the familiar simplicial category $\Delta$ of totally ordered finite sets and increasing maps. Alternatively, $\Lambda$ can be defined by means of its "cyclic covering", the category $E \Lambda$. The latter has one object $(\mathbb{Z}, n)$ for each $n \geq 0$ and the morphisms $f:(\mathbb{Z}, n) \rightarrow$ $(\mathbb{Z}, m)$ are given by non decreasing maps $f: \mathbb{Z} \rightarrow \mathbb{Z}$, such that $f(x+n)=f(x)+m, \quad \forall x \in \mathbb{Z}$. One has $\Lambda=E \Lambda / \mathbb{Z}$, with respect to the obvious action of $\mathbb{Z}$ by translation. To any algebra $\mathcal{A}$ one associates a module $\mathcal{A}^{\natural}$ over the category $\Lambda$ by assigning to each $n$ the $(n+1)$ tensor 
power $\mathcal{A} \otimes \mathcal{A} \cdots \otimes \mathcal{A}$. The cyclic morphisms correspond to the cyclic permutations of the tensors while the face and degeneracy maps correspond to the algebra product of consecutive tensors and the insertion of the unit. The corresponding functor $\mathcal{A} \rightarrow \mathcal{A}^{\natural}$ gives a linearization of the category of associative algebras and cyclic cohomology appears as a derived functor.

One can thus define the distilled module $D(\mathcal{A}, \varphi)$ as the cokernel of the cooling morphism and consider the action of $\mathbb{R}_{+}^{*}$ (obtained from the above equivariance) in the cyclic homology group $H C_{0}(D(\mathcal{A}, \varphi))$. As shown in [66 this in the simplest case of the BC-system gives a cohomological interpretation of the above spectral realization of the zeros of the Riemann zeta function (and of Hecke L-functions).

One striking feature is that the KMS strip ( $c f$. Figure 1) becomes canonically identified in the process with the critical strip of the zeta function (recall that $\beta>1$ ) by multiplication by $i=\sqrt{-1}$.

This cohomological interpretation combines with the above theory of endomotives to give a natural action of the Galois group $G$ of $\overline{\mathbb{Q}} / \mathbb{Q}$ on the above cohomology. This action factorizes to the abelianization $G^{a b}$ and the corresponding decomposition according to characters of $G^{a b}$ corresponds to the spectral realization of L-functions.

The role of the invariant $S(M)$ in the classification of factors or of the more refined flow of weights mentioned above, is very similar to the role of the module of local or global fields and the Brauer theory of central simple algebras. In fact there is a striking parallel (see 66]) between the lattice of unramified extensions $\mathbb{K} \rightarrow \mathbb{K} \otimes_{\mathbb{F}_{q}} \mathbb{F}_{q^{n}}$ of a global field of characteristic $p$ and the lattice of extensions of a factor $M$ by the crossed product algebras $M \rightarrow M \rtimes_{\sigma_{T}} \mathbb{Z}$. Using the algebraic closure of $\mathbb{F}_{q}$ i.e. the operation $\mathbb{K} \rightarrow \mathbb{K} \otimes_{\mathbb{F}_{q}} \overline{\mathbb{F}}_{q}$ corresponds to passing to the dual algebra $M \rightarrow M \rtimes_{\sigma} \mathbb{R}$ and the dual action corresponds to the Frobenius automorphism when as above the appropriate cohomological operations (distillation and $H C_{0}$ ) are performed.

\begin{tabular}{|c|c|}
\hline Global field $\mathbb{K}$ & Factor $M$ \\
\hline $\operatorname{Mod} \mathbb{K} \subset \mathbb{R}_{+}^{*}$ & $\operatorname{Mod} M \subset \mathbb{R}_{+}^{*}$ \\
\hline $\mathbb{K} \rightarrow \mathbb{K} \otimes_{\mathbb{F}_{q}} \mathbb{F}_{q^{n}}$ & $M \rightarrow M \rtimes_{\sigma_{T}} \mathbb{Z}$ \\
\hline$K \rightarrow \mathbb{K} \otimes_{\mathbb{F}_{q}} \overline{\mathbb{F}}_{q}$ & $M \rightarrow M \rtimes_{\sigma} \mathbb{R}$ \\
\hline Points $C\left(\overline{\mathbb{F}}_{q}\right)$ & $\Gamma \subset X_{\mathbb{Q}}$ \\
\hline
\end{tabular}


A notable difference with the original Hilbert space theoretic spectral realization of 64 is that while in the latter case only the critical zeros were appearing directly (the possible noncritical ones appearing as resonances), in the cyclic homology set-up it is more natural to use everywhere the "rapid decay" framework (advocated in [157]) so that all zeros appear on the same footing. This eliminates the difficulty coming from the potential non-critical zeros, so that the trace formula is much easier to prove and reduces to the Riemann-Weil explicit formula. However, it was not obvious how to obtain a direct geometric proof of this formula from the $S$-local trace formula of 64. This was done in [157, showing that the noncommutative geometry framework makes it possible to give a geometric interpretation of the Riemann-Weil explicit formula. While the spectral side of the trace formula is given by the action on the cyclic homology of the distilled space, the geometric side is given as follows 66.

Theorem 26.2. Let $h \in S\left(C_{\mathbb{K}}\right)$. Then the following holds:

$$
\operatorname{Tr}\left(\left.\vartheta(h)\right|_{\mathcal{H}^{1}}\right)=\widehat{h}(0)+\widehat{h}(1)-\Delta \bullet \Delta h(1)-\sum_{v} \int_{\left(\mathbb{K}_{v}^{*}, e_{\mathbb{K}_{v}}\right)}^{\prime} \frac{h\left(u^{-1}\right)}{|1-u|} d^{*} u .
$$

We refer to 66] for the detailed notations which are essentially those of 64. The origin of the terms in the geometric side of the trace formula comes from the Lefschetz formula by AtiyahBott [11] and its adaptation by Guillemin-Sternberg ( $c f$. [110]) to the distribution theoretic trace for flows on manifolds, which is a variation on the theme of [1]. For the action of $C_{\mathbb{K}}$ on the adele class space $X_{\mathbb{K}}$ the relevant periodic orbits on which the computation concentrates turn out to form also the classical points of the noncommutative space $X_{\mathbb{K}} \backslash C_{\mathbb{K}}$ distilled in the above sense from $X_{\mathbb{K}}$. This "classical" subspace of $X_{\mathbb{K}} \backslash C_{\mathbb{K}}$ is given by

$$
\Gamma_{\mathbb{K}}=\cup C_{\mathbb{K}}[v], \quad v \in \Sigma_{\mathbb{K}}
$$

where for each place $v \in \Sigma_{\mathbb{K}}$, one lets $[v]$ be the adele

$$
[v]_{w}=1, \quad \forall w \neq v, \quad[v]_{v}=0 .
$$

In the function field case, one has a non-canonical isomorphism of the following form.

Proposition 26.3. Let $\mathbb{K}$ be the function field of an algebraic curve $C$ over $\mathbb{F}_{q}$. Then the action of the Frobenius on $Y=C\left(\overline{\mathbb{F}}_{q}\right)$ is isomorphic to the action of $q^{\mathbb{Z}}$ on the quotient

$$
\Gamma_{\mathbb{K}} / C_{\mathbb{K}, 1} \text {. }
$$

In the case $\mathbb{K}=\mathbb{Q}$ the space $\Gamma_{\mathbb{Q}} / C_{\mathbb{Q}, 1}$ appears as the union of periodic orbits of period $\log p$ under the action of $C_{\mathbb{Q}} / C_{\mathbb{Q}, 1} \sim \mathbb{R}(c f$. Figure 22). This gives a first approximation to the sought for space $Y=C\left(\overline{\mathbb{F}}_{q}\right)$ in characteristic zero. One important refinement is obtained from the subtle nuance between the adelic description of $X_{\mathbb{Q}}$ and the finer description in terms of the endomotive obtained from the pointed algebraic variety $\left(\mathbb{G}_{m}(\mathbb{Q}), 1\right)$. The second description keeps track of the Galois symmetry and as in proposition 26.3 the isomorphism of the two descriptions is non canonical.

At this point we have, in characteristic zero, several of the geometric notions which are the analogues of the ingredients of Weil's proof and it is natural to try and imitate the steps of 


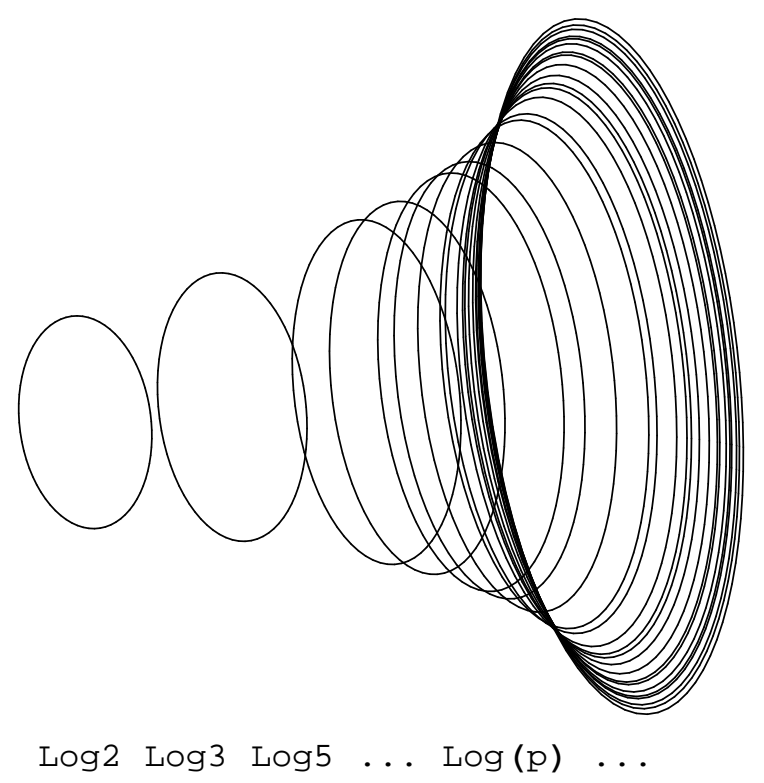

Figure 22. The classical points of the adeles class space

his proof. The step (A), i.e. the explicit formula is taken care of by Theorem 26.2. That what remains is to prove a positivity is a well known result of A. Weil ( $c f$. 24] ) which states that $\mathrm{RH}$ is equivalent to the positivity of the distribution entering in the explicit formulae. Thanks to the above $H^{1}$ obtained as the cyclic homology of the distilled module, Weil's reformulation can be stated as follows.

Theorem 26.4. The following two conditions are equivalent.

- All L-functions with Grössencharakter on $\mathbb{K}$ satisfy the Riemann Hypothesis.

- $\left.\operatorname{Tr} \vartheta\left(f \star f^{\sharp}\right)\right|_{\mathcal{H}^{1}} \geq 0$, for all $f \in \mathcal{S}\left(C_{\mathbb{K}}\right)$.

Here we used the notation

$$
f=f_{1} \star f_{2}, \quad \text { with }\left(f_{1} \star f_{2}\right)(g)=\int f_{1}(k) f_{2}\left(k^{-1} g\right) d^{*} g
$$

for the convolution of functions, using the multiplicative Haar measure $d^{*} g$, and for the adjoint

$$
f \rightarrow f^{\sharp}, \quad f^{\sharp}(g)=|g|^{-1} \bar{f}\left(g^{-1}\right) .
$$

The role of the specific correspondences used in Weil's proof of $\mathrm{RH}$ in positive characteristic is played by the test functions $f \in \mathcal{S}\left(C_{\mathbb{K}}\right)$. More precisely the scaling map which replaces $f(x)$ by $f\left(g^{-1} x\right)$ has a graph, namely the set of pairs $\left(x, g^{-1} x\right) \in X_{\mathbb{K}} \times X_{\mathbb{K}}$, which we view as a correspondence $Z_{g}$. Then, given a test function $f$ on the ideles classes, one assigns to $f$ 
the linear combination

$$
Z(f)=\int f(g) Z_{g} d^{*} g
$$

of the above graphs, viewed as a "divisor" on $X_{\mathbb{K}} \times X_{\mathbb{K}}$.

The analogs of the degrees $d(Z)$ and codegrees $d^{\prime}(Z)=d\left(Z^{\prime}\right)$ of correspondences in the context of Weil's proof are given, for the degree, by

$$
d(Z(h))=\widehat{h}(1)=\int h(u)|u| d^{*} u,
$$

so that the degree $d\left(Z_{g}\right)$ of the correspondence $Z_{g}$ is equal to $|g|$. Similarly, for the codegree one has

$$
d^{\prime}(Z(h))=d\left(Z\left(\bar{h}^{\sharp}\right)\right)=\int h(u) d^{*} u=\widehat{h}(0),
$$

so that the codegree $d^{\prime}\left(Z_{g}\right)$ of the correspondence $Z_{g}$ is equal to 1 .

One of the major difficulties is to find the replacement for the principal divisors which in Weil's proof play a key role as an ideal in the algebra of correspondences on which the trace vanishes. At least already one can see that there is an interesting subspace $V$ of the linear space of correspondences described above on which the trace also vanishes. It is given by the subspace

$$
V \subset \mathcal{S}\left(C_{\mathbb{K}}\right), \quad V=\left\{g(x)=\sum \xi(k x) \mid \xi \in \mathcal{S}\left(\mathbb{A}_{\mathbb{K}}\right)_{0}\right\}
$$

where the subspace $\mathcal{S}\left(\mathbb{A}_{\mathbb{K}}\right)_{0} \subset \mathcal{S}\left(\mathbb{A}_{\mathbb{K}}\right)$ is defined by the two boundary conditions

$$
\xi(0)=0, \quad \int \xi(x) d x=0 .
$$

Lemma 26.5. For any $f \in V \subset \mathcal{S}\left(C_{\mathbb{K}}\right)$, one has

$$
\left.\vartheta(f)\right|_{\mathcal{H}^{1}}=0 .
$$

This shows that the Weil pairing of Theorem 26.4 admits a huge radical given by all functions which extend to adeles and gives another justification for working with the above cohomology $H^{1}$. In particular one can modify arbitrarily the degree and codegree of the correspondence $Z(h)$ by adding to $h$ an element of the radical $V$ using a subtle failure of Fubini's theorem. We will show in a forthcoming paper [67] that several of the steps of Weil's proof can be transposed in the framework described above.

This constitutes a clear motivation to develop noncommutative geometry much further. One can write a very tentative form of a dictionary from the language of algebraic geometry (in the case of curves) and that of noncommutative geometry. The dictionary is summarized in the following table. It should be stressed that the main problem is to find the correct translation in the right column (non-commutative geometry) of the well established notion of principal divisor in the (algebraic geometry) left column. The table below is too rough in that respect since one does not expect to be able to work in the usual "primary" theory which involves periodic cyclic homology and index theorems. Instead one expects that both the unstable cyclic homology and the finer invariants of spectral triples arising from transgression will play 
an important role. Thus the table below should be taken as a very rough first approximation, and a motivation for developing the missing finer notions in the right column.

\begin{tabular}{|c|c|}
\hline Virtual correspondences & bivariant $K$-theory class $\Gamma$ \\
\hline Modulo torsion & $K K\left(A, B \otimes \mathrm{II}_{1}\right)$ \\
\hline Effective correspondences & Epimorphism of $C^{*}$-modules \\
\hline Principal correspondences & Compact morphisms \\
\hline Composition & cup product in $K K$-theory \\
\hline Degree of correspondence & Pointwise index $d(\Gamma)$ \\
\hline $\operatorname{deg} D(P) \geq g \Rightarrow \sim$ effective & $d(\Gamma)>0 \Rightarrow \exists K, \Gamma+K$ onto \\
\hline $\begin{array}{l}\text { Adjusting the degree } \\
\text { by trivial correspondences }\end{array}$ & $\begin{array}{l}\text { Fubini step } \\
\text { on the test functions }\end{array}$ \\
\hline Frobenius correspondence & Correspondence $Z_{g}$ \\
\hline Lefschetz formula & $\begin{array}{c}\text { bivariant Chern of } Z(h) \\
\text { (localization on graph } Z(h) \text { ) }\end{array}$ \\
\hline $\begin{array}{l}\text { Weil trace unchanged } \\
\text { by principal divisors }\end{array}$ & $\begin{array}{l}\text { bivariant Chern unchanged } \\
\text { by compact perturbations }\end{array}$ \\
\hline
\end{tabular}




\section{REFERENCES}

[1] J. Aastrup, R. Nest, E. Schrohe, A Continuous Field of $C^{*}$-algebras and the Tangent Groupoid for Manifolds with Boundary, ArXiv math.FA/0507317.

[2] B. Abadie, R. Exel, Hilbert $C^{*}$-bimodules over commutative $C^{*}$-algebras and an isomorphism condition for quantum Heisenberg manifolds, Rev. Math. Phys. Vol.9 (1997) no. 4, 411-423.

[3] B. Abadie, S. Eilers, R. Exel, Morita equivalence for crossed products by Hilbert $C^{*}$-bimodules. Trans. Amer. Math. Soc. Vol.350, (1998) no. 8, 3043-3054.

[4] J.E. Anderson, I.F. Putnam, Topological invariants for substitution tilings and their associated $C^{*}$ algebras. Ergodic Theory Dynam. Systems 18 (1998), no. 3, 509-537.

[5] Anonymous Persian manuscript, Fi tadakhul al-ashkal al-mutashabiha aw mutawafiqa, Bibliothèque Nationale, Paris, Ancien fonds persan 169.

[6] C. Antonescu, E. Christensen, Spectral triples for $A F C^{*}$-algebras and metrics on the Cantor set, preprint arXiv math.OA/0309044

[7] Farhad Ardalan, Amir H. Fatollahi, Point-Like Structure in Strings and Non-Commutative Geometry, Phys.Lett. B408 (1997) 157-163.

[8] F. Ardalan, H. Arfaei, N. Sadooghi, On the Anomalies and Schwinger Terms in Noncommutative Gauge Theories, hep-th/0507230

[9] Nader Ardalan and Laleh Bakhtiar, The sense of unity: the Sufi tradition in Persian architecture, The University of Chicago Press, 1973.

[10] M. Artin, J. Tate, and M. Van den Bergh, Some algebras associated to automorphisms of elliptic curves. The Grothendieck Festschrift. vol.I. Prog. Math., Vol.86:33-85, 1990.

[11] M.F. Atiyah and R. Bott, A Lefschetz fixed point formula for elliptic complexes: I, Annals of Math, Vol.86 (1967) 374-407.

[12] M.F. Atiyah, Global theory of elliptic operators. Proc. Internat. Conf. on Functional Analysis and Related Topics (Tokyo, 1969). University of Tokyo press, Tokyo 1970, 21-30.

[13] Michael Baake and Robert V. Moody (Editors), Directions in mathematical quasicrystals, CRM Monograph Series, 13. American Mathematical Society, Providence, RI, 2000. viii+379 pp.

[14] T. Banks, W. Fischler, S.H. Shenker, L. Susskind, M theory as a Matrix model: a conjecture, Phys.Rev. D55 (1997) 5112-5128. hep-th/9610043

[15] P. Baum and A. Connes, Geometric K-theory for Lie groups and foliations, Preprint IHES (M/82/), 1982, L'enseignement Mathematique, t.46 (2000), 1-35.

[16] P. Baum, A. Connes, and N. Higson, Classifying space for proper-actions and K-theory of group $C^{*}$ algebras, Contemp. Math. Vol.167 (1994), 241-291.

[17] F. Bayen and C. Fronsdal. Quantization on the sphere. J. Math. Phys. Vol.22 (1981) N.7, $1345-1349$.

[18] J. Bellissard, Noncommutative geometry and quantum Hall effect. In: Proceedings of the International Congress of Mathematicians, Vol. 1, 2 (Zürich, 1994), pages 1238-1246, Basel, 1995. Birkhäuser.

[19] J. Bellissard, A. van Elst, and H. Schulz-Baldes. The noncommutative geometry of the quantum Hall effect. J. Math. Phys., 35 (1994) N.10, 5373-5451.

[20] J. Bellissard, The noncommutative geometry of aperiodic solids, in "Geometric and topological methods for quantum field theory (Villa de Leyva, 2001)", 86-156, World Scientific, 2003.

[21] M-T. Benameur, T. Fack, On von Neumann spectral triples, math.KT/0012233

[22] R. Berger, M. Dubois-Violette, M. Wambst, Homogeneous algebras, math.QA/0203035

[23] C.G. Bollini, J.J. Giambiagi Lowest order divergent graphs in $\nu$-dimensional space Phys.Lett. B40 (1972) $566-568$.

[24] E. Bombieri, Problems of the Millenium: The Riemann Hypothesis, Clay mathematical Institute (2000).

[25] M. Born and P. Jordan. Zur Quantenmechanik, Z. Phys., 34 (1925) 858-888.

[26] D. Borthwick, S. Klimek, A. Lesniewski, M. Rinaldi, Supersymmetry and Fredholm modules over quantized spaces. Comm. Math. Phys. 166 (1994), no. 2, 397-415.

[27] J.-B. Bost and A. Connes, Hecke Algebras, Type III factors and phase transitions with spontaneous symmetry breaking in number theory, Selecta Mathematica, New Series Vol.1, N.3 (1995), 411-457.

[28] P. Bouwknegt, K. Hannabuss, V. Mathai, T-duality for principal torus bundles. J. High Energy Phys. 2004, no. 3, 018, 10 pp. (electronic). 
[29] P. Bouwknegt, J. Evslin, V. Mathai, T-duality: topology change from H-flux. Comm. Math. Phys. 249 (2004), no. 2, 383-415.

[30] M. Boyle, D. Handelman, Orbit equivalence, flow equivalence, and ordered cohomology, Israel J. Math. 95 (1996) 169-210.

[31] O. Bratteli. Inductive limits of finite dimensional $C^{*}$-algebras. Trans. Amer. Math. Soc., 171 (1972) 195234.

[32] P. Breitenlohner, D. Maison, Dimensional renormalization and the action principle. Comm. Math. Phys. Vol.52 (1977), N.1, 11-38.

[33] J. Brüning, R. Seeley, Regular singular asymptotics, Adv. in Math. 58 (1985) 133-148.

[34] J-L. Brylinski and V. Nistor, Cyclic Cohomology of Etale Groupoids, K-theory, Vol.8 (1994), 341-365.

[35] M.S. Bulatov, Geometricheskaya garmonizatsiya v arkhitekture Srednei Azii IX-XV vv., Nauka, 2nd ed., Moskva 1988, $360 \mathrm{pp}$.

[36] D. Burghelea, The cyclic homology of the group rings, Comment. Math. Helv. Vol.60 (1985) 354-365.

[37] A. Carey, K. Hannabuss, V. Mathai, P. McCann, Quantum Hall Effect on the hyperbolic plane, Commun. Math. Physics, Vol.190, no. 3 (1998) 629-673.

[38] Alan L. Carey, J. Phillips, A. Rennie, F. A. Sukochev The Local Index Formula in Semifinite von Neumann Algebras I: Spectral Flow, math.OA/0411021

[39] Alan L. Carey, J. Phillips, A. Rennie, F. A. Sukochev The Local Index Formula in Semifinite von Neumann Algebras II: The Even Case, math.OA/0411021

[40] J.M. Castera, Zellijs, muqarnas and quasicrystals, ISAMA 99 Proceedings, pp.99-104, 1999.

[41] J.M. Castera, Arabesques, ACR editions, 1999, 486 pp.

[42] P.S. Chakraborti, A. Pal, Equivariant spectral triple on the quantum SU(2)-group, K-Theory 28 (2003), no. 2, 107-126.

[43] T. Chakraborti, P. Pietilänen, The Quantum Hall Effects, Second Edition, Springer 1995.

[44] A. Chamseddine and A. Connes, Universal formulas for noncommutative geometry actions, Phys. Rev. Letters, Vol.77, N.24, (1996) 4868-4871.

[45] A. Chamseddine and A. Connes, The spectral action principle, Commun. Math. Phys. 186 (1997) 731-750.

[46] A. Chamseddine and A. Connes, Scale Invariance in the spectral action, Arxiv hep-th/0512169

[47] J. Cheeger, M. Gromov, $L^{2}$-cohomology and group cohomology, Topology, Vol.25 (1986), 189-215.

[48] A. Chou, The Dirac Operator on Spaces with Conical Singularities and Positive Scalar Curvatures, Trans. Am. Math. Soc. 289 (1985), no. 1, 1-40.

[49] E. Christensen, C. Ivan, Sums of two dimensional spectral triples, ArXiv math.OA/0601024

[50] J. Collins, Renormalization, Cambridge Monographs in Math. Physics, Cambridge University Press, 1984.

[51] A. Connes, Une classification des facteurs de type III, Ann. Sci. Ecole Norm. Sup., 6, n. 4 (1973), $133-252$.

[52] A. Connes, Sur la théorie non commutative de l'intégration Algèbres d'opérateurs (Sém., Les Plans-surBex, 1978), p. 19-143, Lecture Notes in Math., 725, Springer, Berlin, 1979.

[53] A. Connes, Géométrie Noncommutative. InterEditions, Paris, 1990.

[54] A. Connes, Noncommutative geometry. Academic Press, 1994.

[55] A. Connes, An analogue of the Thom isomorphism for crossed products of a $C^{*}$-algebra by an action of $\mathbb{R}$, Adv. in Math. 39 (1981), no. 1, 31-55.

[56] A. Connes, Cyclic cohomology and the transverse fundamental class of a foliation. In: Geometric methods in operator algebras (Kyoto, 1983). Pitman Res. Notes in Math., 123, Longman, Harlow 1986, 52-144.

[57] A. Connes, $C^{*}$ algèbres et géométrie differentielle. C.R. Acad. Sci. Paris, Ser. A-B , 290 (1980) 599-604.

[58] A. Connes, Noncommutative differential geometry. Inst. Hautes Etudes Sci. Publ. Math., 62, 1985, 257360.

[59] A. Connes, Compact metric spaces, Fredholm modules, and hyperfiniteness, Ergod. Th. Dynam. Sys. (1989) 9, 207-220.

[60] A. Connes, On the Chern character of $\theta$-summable Fredholm modules Comm. Math. Phys. 139 (1991), n 1, p. 171-181.

[61] A. Connes, Gravity coupled with matter and foundation of noncommutative geometry. Commun. Math. Phys., 182 (1996) 155-176.

[62] A. Connes, Noncommutative geometry and reality. Journal of Math. Physics, 36, n.11 (1995) 6194-6231.

[63] A. Connes, Geometry from the spectral point of view. Lett. Math. Phys. 34 (1995), no. 3, 203-238. 
[64] A. Connes, Trace formula in Noncommutative Geometry and the zeros of the Riemann zeta function. Selecta Mathematica. New Ser. 5 (1999) 29-106.

[65] A. Connes, Cyclic cohomology, quantum group symmetries and the local index formula for $\mathrm{SU}_{q}(2)$. J. Inst. Math. Jussieu 3 (2004), no. 1, 17-68.

[66] A. Connes, C. Consani, M. Marcolli, Noncommutative geometry and motives: the thermodynamics of endomotives, math.QA/0512138

[67] A. Connes, C. Consani, M. Marcolli, The Weil proof and the geometry of the adeles class space, in preparation.

[68] A. Connes, M. Douglas, A. Schwarz, Noncommutative geometry and Matrix theory: compactification on tori. J. High Energy Phys. (1998) no. 2, Paper 3, 35 pp. (electronic)

[69] A. Connes, M. Dubois-Violette, Noncommutative finite-dimensional manifolds. I. Spherical manifolds and related examples. Comm. Math. Phys. 230 (2002), no. 3, 539-579.

[70] A. Connes, M. Dubois-Violette, Moduli space and structure of noncommutative 3-spheres. Lett. Math. Phys. 66 (2003), no. 1-2, 91-121.

[71] A. Connes, M. Dubois-Violette, Non commutative finite-dimensional manifolds II. Moduli space and structure of noncommutative 3-spheres. ArXiv math.QA/0511337

[72] A. Connes and G. Landi, Noncommutative manifolds, the instanton algebra and isospectral deformations. Comm. Math. Phys. 221 (2001), no. 1, 141-159.

[73] A. Connes, J. Lott, Particle models and noncommutative geometry, in "Recent advances in field theory" (Annecy-le-Vieux, 1990). Nuclear Phys. B Proc. Suppl. 18B (1990), 29-47 (1991).

[74] A. Connes, M. Marcolli, From Physics to Number Theory via Noncommutative Geometry. Part I: Quantum Statistical Mechanics of Q-lattices, to appear in the volume "Frontiers in Number Theory Physics and Geometry" math.NT/0404128

[75] A. Connes, M. Marcolli, $\mathbb{Q}$-lattices: quantum statistical mechanics and Galois theory, Journal of Geometry and Physics, Vol. 56 (2005) N.1, 2-23.

[76] A. Connes, M. Marcolli, Anomalies, Dimensional Regularization and Noncommutative Geometry, in preparation.

[77] A. Connes, M. Marcolli, Noncommutative geometry, from quantum fields to motives (tentative title), book in preparation. To appear as a co-publication of the American Mathematical Society, Colloquium Publications Series, and Hindustan Book Agency, Texts and Readings in Mathematics Series.

[78] A. Connes, M. Marcolli, N. Ramachandran, KMS states and complex multiplication, to appear in Selecta Mathematica.

[79] A. Connes, M. Marcolli, N. Ramachandran, KMS states and complex multiplication. Part II, to appear in the proceedings of the Abel Symposium 2005.

[80] A. Connes, H. Moscovici, Cyclic cohomology, the Novikov conjecture and hyperbolic groups, Topology, Vol. 29 (1990) no. 3, 345-388.

[81] A. Connes and H. Moscovici, The local index formula in noncommutative geometry. GAFA, 5 (1995) $174-243$.

[82] A. Connes and H. Moscovici, Hopf Algebras, Cyclic Cohomology and the Transverse Index Theorem. Commun. Math. Phys. 198 (1998) 199-246.

[83] A. Connes and H. Moscovici, Differentiable cyclic cohomology and Hopf algebraic structures in transverse geometry. Essays on geometry and related topics, Vol. 1, 2, 217-255, Monogr. Enseign. Math., 38, Enseignement Math., Geneva, 2001.

[84] A. Connes and H. Moscovici, Modular Hecke algebras and their Hopf symmetry, Mosc. Math. J. 4 (2004), no. 1, 67-109, 310 (math.QA/0301089).

[85] A. Connes and H. Moscovici, Rankin-Cohen Brackets and the Hopf Algebra of Transverse Geometry, Mosc. Math. J. 4 (2004), no. 1, 111-130, 311. (math.QA/0304316).

[86] A. Connes and H. Moscovici, Background independent geometry and Hopf cyclic cohomology, math.QA/0505475

[87] A. Connes and H. Moscovici, Spectral Geometry of Noncommutative Spaces (tentative title), book in preparation.

[88] A. Connes and M. Rieffel, Yang-Mills for noncommutative two tori. Contemp. Math. Oper. Algebra Math. Phys. AMS (1987) 237-266. 
[89] A. Connes and G. Skandalis, The longitudinal index theorem for foliations Publ. Res. Inst. Math. Sci. 20 (1984), n 6, p. 1139-1183.

[90] A. Connes and M. Takesaki, The flow of weights on factors of type III, Tohoku Math. J. 29 (1977), 473-575.

[91] C. Consani, M. Marcolli, Noncommutative geometry, dynamics, and $\infty$-adic Arakelov geometry. Selecta Math. (N.S.) 10 (2004), no. 2, 167-251.

[92] C. Consani, M. Marcolli, New perspectives in Arakelov geometry. Number theory, 81-102, CRM Proc. Lecture Notes, 36, Amer. Math. Soc., Providence, RI, 2004.

[93] M. Crainic, Cyclic Cohomology of Etale Groupoids, the general case, K-theory, Vol.17 (1999), 319-362.

[94] J. Cuntz, A class of $C^{*}$-algebras and topological Markov chains II: reducible chains and the Ext-functor for $C^{*}$-algebras, Invent. Math. 63 (1981) 25-40.

[95] J. Cuntz, W. Krieger, A class of $C^{*}$-algebras and topological Markov chains, Invent. Math. 56 (1980) $251-268$

[96] K.R. Davidson, $C^{*}$-algebras by example. American Mathematical Society, Providence, RI, 1996.

[97] J. Dixmier, Existence de traces non normales. C.R. Acad. Sci. Paris, Ser. A-B, 262 (1966) A1107-A1108.

[98] M. Dubois-Violette, Generalized differential spaces with $d^{N}=0$ and the q-differential calculus, Czech J. Phys. 46 (1997) 1227-1233.

[99] M. Dubois-Violette, $d^{N}=0$ : Generalized homology, K-Theory, 14 (1998) 371-404.

[100] M. Dubois-Violette, Lectures on graded differential algebras and noncommutative geometry, In Y. Maeda and H. Moriyoshi, editors, Noncommutative Differential Geometry and Its Applications to Physics, pages 245-306. Shonan, Japan, 1999, Kluwer Academic Publishers, 2001.

[101] M. Dubois-Violette and M. Henneaux, Generalized cohomology for irreducible tensor fields of mixed Young symmetry type Lett. Math. Phys. 49 (1999) 245-252.

[102] M. Dubois-Violette and M. Henneaux, Tensor fields of mixed Young symmetry type and N-complexes, Commun. Math. Phys. 226 (2002) 393-418.

[103] H. Figueroa, J.M. Gracia-Bondía, J. Varilly, Elements of Noncommutative Geometry, Birkhäuser, 2000.

[104] D. Gaboriau, Invariants $\ell^{2}$ de relations d'équivalence et de groupes, Publ. Math. Inst. Hautes Études Sci. No. 95 (2002), 93-150.

[105] D. Gaboriau, Approximate dimension for equivalence relations and groups, in preparation.

[106] V. Gayral, J.M. Gracia-Bondía, B. Iochum, T. Schücker, J.C. Várilly, Moyal planes are spectral triples. Comm. Math. Phys. 246 (2004), no. 3, 569-623.

[107] T. Giordano, I. F. Putnam and C. Fr. Skau, Topological orbit equivalence and $C^{*}$-crossed products. J. Reine Angew. Math. 469 (1995), 51-111

[108] D. Guido, T. Isola, Dimensions and singular traces for spectral triples, with applications to fractals, Journ. Funct. Analysis, 203 (2003), 362-400. (math.OA/0202108)

[109] D. Guido, T. Isola, Dimensions and spectral triples for fractals in $R^{N}$, math.OA/0404295

[110] V. Guillemin, Lectures on spectral theory of elliptic operators, Duke Math. J., Vol.44 (1977) N.3, $485-517$.

[111] D. Gurevich and L. Vainerman, Non-commutative analogues of q-special polynomials and a q-integral on a quantum sphere. J. Phys. A, 31 (1998) 7, 1771-1780.

[112] E. Ha, F. Paugam, Bost-Connes-Marcolli systems for Shimura varieties. I. Definitions and formal analytic properties, IMRP 2005, Vol.5 (2005) 237-286. (math.OA/0507101)

[113] P.M. Hajac, M. Khalkhali, B. Rangipour, Y. Sommerhaeuser, Hopf-cyclic homology and cohomology with coefficients. math.KT/0306288

[114] E.H. Hall, On a new action of the magnet on electric currents, Amer. J. of Math. 2 (1879) 287.

[115] E. Hawkins, Hamiltonian gravity and noncommutative geometry. Comm. Math. Phys. 187 (1997), no. 2, 471-489.

[116] W. Heisenberg, Über quantentheoretische Umdeutung kinematischer und mechanischer Beziehungen, Z. Phys. 33 (1925) 879-893.

[117] G. Hochschild, B. Kostant, A. Rosenberg, Differential forms on regular affine algebras. Trans. Amer. Math. Soc. Vol.102 (1962) 383-408.

[118] G. 't Hooft, M. Veltman, Regularization and renormalization of gauge fields Nuclear Physics B, Vol.44, N.1 (1972), 189-213. 
[119] S. Hurder, Secondary classes and the von Neumann algebra of a foliation, Preprint MSRI Berkeley (1983).

[120] A. Jaffe, A. Lesniewski, K. Osterwalder, Quantum K-theory : the Chern character, Commun. Math. Phys., 118 (1988) 1-14.

[121] P. Julg, Travaux de N. Higson et G. Kasparov sur la conjecture de Baum-Connes. Seminaire Bourbaki, (1997-98), Expose 841, Vol.252 (1998), 151-183.

[122] W. Kalau and M. Walze, Gravity, noncommutative geometry and the Wodzicki residue. J. of Geom. and Phys., 16 (1995) 327-344.

[123] G.G. Kasparov, The operator K-functor and extensions of $C^{*}$ algebras. Izv. Akad. Nauk SSSR, Ser. Mat., 44 (1980) 571-636; Math. USSR Izv., 16 (1981) 513-572.

[124] D. Kastler, The Dirac operator and gravitation. Commun. Math. Phys., 166 (1995) 633-643.

[125] D. Kastler, A detailed account of Alain Connes' version of the standard model in noncommutative geometry. I, II, Rev. Math. Phys. 5 (1993), no. 3, 477-532. III, Rev. Math. Phys. 8 (1996), no. 1, $103-165$.

[126] M. Khalkhali, Very Basic Noncommutative Geometry, arXiv:math.KT/0408416 Lecture Notes Series IPM Tehran, Vol.5, 2005.

[127] M. Khalkhali and B. Rangipour, A new cyclic module for Hopf algebras, K-Theory 27 (2002), no. 2, $111-131$

[128] M. Khalkhali and B. Rangipour, Invariant cyclic homology, K-Theory 28 (2003), no. 2, $183-205$.

[129] M. Khalkhali and B. Rangipour, Cyclic cohomology of (extended) Hopf algebras, Noncommutative geometry and quantum groups, (Warsaw, 2001), 59-89, Banach Center Publ., 61, Polish Acad. Sci., Warsaw, 2003.

[130] A. Klimyk and K. Schmuedgen, Quantum groups and their representations, Springer Verlag, 1998.

[131] K. von Klitzing, G. Dorda, M. Pepper, New method for high-accuracy determination of the fine-structure constant based on quantized hall resistance. Phys. Rev. Lett. 45 (1980) N.6, 494-497.

[132] M. Kontsevich, Deformation quantization of Poisson manifolds I, IHES preprint M/97/72.

[133] M. Kontsevich, Formal (non) commutative symplectic geometry. In: The Gel'fand Mathematical Seminars, 1990-1992, pages 173-187. Birkhäuser Boston, Boston, MA, 1993.

[134] M. Kontsevich and A.L. Rosenberg, Noncommutative smooth spaces. In: The Gelfand Mathematical Seminars, 1996-1999, pages 85-108. Birkhäuser Boston, Boston, MA, 2000.

[135] V. Lafforgue, K-théorie bivariante pour les algèbres de Banach et conjecture de Baum-Connes, Inv. Math. Vol.149 (2002), 1-97.

[136] G. Landi, An Introduction to Noncommutative Spaces and their Geometry. Lecture Notes in Physics: Monographs m51, Springer-Verlag, 1997. ISBN 3-540-63509-2.

[137] M.L. Lapidus, M. van Frankenhuysen, Fractal geometry and number theory. Complex dimensions of fractal strings and zeros of zeta functions. Birkhäuser Boston, Inc., Boston, MA, 2000.

[138] B. Laughlin, Quantized Hall conductivity in two dimensions. Phys. Rev. B, 23 (1981) 5632.

[139] H. Laurent, Sur la théorie des nombres premiers. C.R. Acad. Sci. Paris, 126 (1898) 809-810.

[140] M. Lesch, Operators of Fuchs type, conical singularities, and asymptotic methods, B. G. Teubner Verlagsgesellschaft mbH, Stuttgart, 1997.

[141] J-M. Lescure, Triplets spectraux pour les variétés á singularité conique isolée. Bull. Soc. Math. France 129 (2001), no. 4, 593-623.

[142] Yu.I. Manin, The notion of dimension in geometry and algebra, to appear in Bulletin of the AMS (math.AG/0502016).

[143] Yu.I. Manin, Real multiplication and noncommutative geometry, The legacy of Niels Henrik Abel, 685727, Springer, Berlin, 2004. (math.AG/0202109)

[144] Yu.I. Manin, Topics in noncommutative geometry. Princeton University Press, Princeton, NJ, 1991.

[145] Yu.I. Manin, Three-dimensional hyperbolic geometry as $\infty$-adic Arakelov geometry, Invent. Math. 104 (1991) 223-244.

[146] Yu.I. Manin, Quantum groups and noncommutative geometry. Centre Recherche Math. Univ. Montréal 1988.

[147] Yu.I. Manin, M. Marcolli, Continued fractions, modular symbols, and noncommutative geometry. Selecta Math. (N.S.) 8 (2002), no. 3, 475-521. 
[148] Yu.I. Manin, A.A. Panchishkin, Introduction to modern number theory. Fundamental problems, ideas and theories. Second edition. Encyclopaedia of Mathematical Sciences, 49. Springer-Verlag, 2005. xvi+514 pp.

[149] M. Marcolli, Arithmetic noncommutative geometry, University Lecture Series, 36. American Mathematical Society, 2005. xii+136 pp.

[150] M. Marcolli, Limiting modular symbols and the Lyapunov spectrum. J. Number Theory 98 (2003), no. 2, $348-376$.

[151] M. Marcolli, Modular curves, $C^{*}$-algebras, and chaotic cosmology, math-ph/0312035

[152] M. Marcolli and V. Mathai, Twisted index theory on good orbifolds, II: fractional quantum numbers, Communications in Mathematical Physics, Vol.217, no.1 (2001) 55-87.

[153] M. Marcolli and V. Mathai, Twisted index theory on good orbifolds, I: noncommutative Bloch theory, Communications in Contemporary Mathematics, Vol.1 (1999) 553-587.

[154] M. Marcolli and V. Mathai, Towards the fractional quantum Hall effect: a noncommutative geometry perspective, cond-mat/0502356

[155] V. Mathai, J. Rosenberg, T-duality for torus bundles with H-fluxes via noncommutative topology. Comm. Math. Phys. 253 (2005), no. 3, 705-721

[156] J. Mather, Commutators of diffeomorphisms. II, Comment. Math. Helv. 50 (1975), 33-40.

[157] R. Meyer, On a representation of the idele class group related to primes and zeros of L-functions, preprint arXiv math.NT/0311468

[158] J.S. Milne, Shimura varieties: the geometric side of the zeta function, preprint available at www.jmilne.org

[159] J. Milnor and D. Stasheff, Characteristic classes. Ann. of Math. Stud., Princeton University Press, Princeton, N.J. 1974.

[160] B. Monthubert, Pseudodifferential calculus on manifolds with corners and groupoids Proc. Amer. Math. Soc. 127 (1999) no 10, 2871-2881. ArXiv math.KT/0507601

[161] B. Monthubert and V. Nistor, A topological index theorem for manifolds with corners

[162] D. Nagy, Visual mathematics: a missing link in a split culture. Vis. Math. 1 (1999), no. 2, 20 pp. (electronic).

[163] N. Nekrasov and A. Schwarz, Instantons in noncommutative $\mathbb{R}^{4}$ and (2,0) superconformal six dimensional theory. Comm. Math. Phys. 198 (1998), no. 3, 689-703.

[164] W. Parry, S. Tuncel, Classification problems in ergodic theory, London Math. Soc. Lecture Notes Series 67, 1982.

[165] R. Penrose, Pentaplexity: a class of nonperiodic tilings of the plane. Math. Intelligencer 2 (1979/80), no. $1,32-37$.

[166] M. V. Pimsner, A class of $C^{*}$-algebra generalizing both Cuntz-Krieger algebras and crossed products by $Z$. Free probablility Theory, 189-212. Fields Inst. Comm. Vol.12, American Math. Society, 1997.

[167] M. Pimsner and D. Voiculescu, Exact sequences for $K$ groups and Ext group of certain crossed product $C^{*}$-algebras. J. Operator Theory, 4 (1980) 93-118.

[168] A. Polishchuk, Noncommutative two-tori with real multiplication as noncommutative projective varieties. J. Geom. Phys. 50 (2004), no. 1-4, 162-187.

[169] M. Puschnigg, Characters of Fredholm modules and a problem of Connes, Preprint 2005.

[170] I. Putnam, $C^{*}$-algebras from Smale spaces, Can. J. Math. 48 (1996) N.1 175-195.

[171] I. Putnam, J. Spielberg, The structure of $\mathrm{C}^{*}$-algebras associated with hyperbolic dynamical systems, J. Funct. Anal. 163 (1999) 279-299.

[172] J-P. Ramis, Séries divergentes et théories asymptotiques. Bull. Soc. Math. France, 121(Panoramas et Syntheses, suppl.) 74, 1993.

[173] J. Renault, A groupoid approach to $C^{*}$-algebras. Lecture Notes in Math. 793, Springer, Berlin, 1980.

[174] M.A. Rieffel, $C^{*}$-algebras associated with irrational rotations. Pacific J. Math., 93 (1981) 415-429.

[175] M.A. Rieffel, The cancellation theorem for projective modules over irrational rotation $C^{*}$-algebras. Proc. London Math. Soc., 47 (1983) 285-302.

[176] M.A. Rieffel, Deformation quantization of Heisenberg manifolds. Comm. Math. Phys. 122 (1989) 4, 531-562

[177] G. Robertson, Boundary actions for affine buildings and higher rank Cuntz-Krieger algebras, in J. Cuntz, S. Echterhoff (eds.) "C*-algebras", Springer Verlag 2001, pp. 182-202. 
[178] A.L. Rosenberg, Noncommutative schemes. Compositio Math. 112 (1998) N.1, 93-125.

[179] A.L. Rosenberg. Noncommutative algebraic geometry and representations of quantized algebras. Kluwer Academic Publishers Group, Dordrecht, 1995.

[180] D. Ruelle, Non-commutative algebras for hyperbolic diffeomorphisms, Invent. Math. 93 (1988) 1-13.

[181] T. Schucker, Spin group and almost commutative geometry. preprint hep-th/0007047

[182] N. Seiberg and E. Witten, String theory and noncommutative geometry. J. High Energy Phys. 1999 , no. 9, Paper 32, 93 pp.

[183] M.M. Sheikh-Jabbari, Tiny Graviton Matrix Theory: DLCQ of IIB Plane-Wave String Theory, A Conjecture, JHEP 0409 (2004) 017. hep-th/0406214

[184] G. Shimura, Arithmetic theory of automorphic functions, Iwanami Shoten and Princeton 1971.

[185] G. Skandalis, Approche de la conjecture de Novikov par la cohomologie cyclique. Seminaire Bourbaki, (1990-91), Expose 739, Astérisque No.201-203 (1992), 299-320.

[186] G. Skandalis, Progrès récents sur la conjecture de Baum-Connes. Contribution de Vincent Lafforgue, Séminaire Bourbaki, Vol.1999-2000, Expose 869, Astérisque No. 276 (2002), 105-135.

[187] C. Skau, Orbit structure of topological dynamical systems and its invariants, Operator algebras and quantum field theory (Rome, 1996), 533-544, Internat. Press, Cambridge, MA, 1997.

[188] S.P. Smith and J.T. Stafford. Regularity of the four dimensional Sklyanin algebra. Compos. Math., Vol.83 (1992) 259-289.

[189] Y. Soibelman, Quantum tori, mirror symmetry and deformation theory. Lett. Math. Phys. 56 (2001) 99-125.

[190] J. Spielberg, Free-product groups, Cuntz-Krieger algebras, and covariant maps. Internat. J. Math. 2 (1991), no. 4, 457-476.

[191] W. van Suijlekom, L. Dabrowski, G. Landi, A. Sitarz, J.C. Varilly, Local index formula for $S U_{q}(2)$, math.QA/0501287

[192] W. van Suijlekom, L. Dabrowski, G. Landi, A. Sitarz, J.C. Varilly, The Dirac operator on $S U_{q}(2)$, math.QA/0411609

[193] D. Sullivan, Geometric periodicity and the invariants of manifolds. Lecture Notes in Math., 197, Springer, 1971.

[194] T. Sunada, A discrete analogue of periodic magnetic Schrödinger operators, Contemp. Math. Vol.173 (1994) 283-299.

[195] M. Takesaki, Tomita's theory of modular Hilbert algebras and its applications. Lecture Notes in Math., 28, Springer, 1970.

[196] M. Veltman, Diagrammatica, Cambridge University Press, 1994.

[197] B.L. van der Waerden, Sources of Quantum Mechanics. Dover, New York, 1967.

[198] Abu'l-Wafa' al-Buzjani, Kitab fima yahtaju ilayhi al-sani' min a'mal al-handasa. Milan manuscript (Arabic): Biblioteca Ambrosiana, Arab. 68.

[199] A. Wassermann, Ergodic actions of compact groups on operator algebras. III. Classification for $\mathrm{SU}(2)$. Invent. Math. 93 (1988) 2, 309-354.

[200] H.E. Winkelnkemper, The graph of a foliation, Ann. Global Anal. Geom. 1 (1983), no 3, 51-75.

[201] E. Witten, Bound states of strings and p-Branes, Nuclear Physics B 460 (1996) 335-360.

[202] M. Wodzicki, Noncommutative residue, Part I. Fundamentals. In K-theory, arithmetic and geometry. Lecture Notes in Math., 1289, Springer, Berlin 1987.

[203] D. Zagier, Modular forms and differential operators, Proc. Indian Acad. Sci. Math. Sci. (K.G.Ramanathan memorial issue) 104 (1994) N.1, 57-75.

A. Connes: Collège de France, 3, Rue D'Ulm, Paris, F-75005 France, I.H.E.S. And Vanderbilt UNIVERSITY

E-mail address: alain@connes.org

M. Marcolli: Max-Planck Institut für Mathematik, Vivatsgasse 7, Bonn, D-53111 Germany

E-mail address: marcolli@mpim-bonn.mpg.de 